\title{
Energy Restoration in Mobile Sensor Networks in the Presence of Physical Obstacles
}

\author{
BY \\ Agustin Magrans Rico \\ A thesis submitted to the Faculty of Graduate And \\ Post-Doctoral AfFAirs in PARTial FUlfilment of THE \\ REQUIREMENTS FOR THE DEGREE OF \\ Master of Computer Science
}

School of Computer Science

Carleton University

Ottawa, Ontario

(C) 2017

Agustin Magrans Rico 


\section{Acknowledgements}

I would like to express my sincere and deep gratitude to my supervisor Nicola Santoro and my co-supervisor Elio Velazques for their infinite patience, the continuous support, and for all the knowledge they shared with me. Thank you very much.

I am very grateful for all the love, patience, and encourage that Glenda gives me every single day. You are the best of my life. Thank for believing in me and for supporting my decisions.

Finally, I would like to thank my family and friends for the love, and support they shared with me. 


\begin{abstract}
The problem of how to restore mobile sensors' energy efficiently has become a very important field of investigation in Mobile Sensor Network (MSN). The main goal of any energy restoration technique is to reach a state where the continuous operation of the sensor network can be guaranteed and the number of sensor losses can be limited in scope and time. The present thesis analyzes the impact of adding physical obstacles to the MSN scenarios. It is proposed that an efficient obstacle avoidance technique could be introduced that allows sensors to collect important information about the obstacles. This information could be used by the sensors to improve their paths and avoid unnecessary exploration. Different energy restoration techniques in presence of physical obstacles are compared. It is shown in the simulation results that our proactive strategy, which uses information about the obstacles to improving the paths around them, achieves the best results.
\end{abstract}




\section{List of Acronyms}

CDG Compass Direct Graph

IBA Intelligent Bug Algorithm

FFP Frugal Feeding Problem

FGM Follow the Gap Method

FOV Field Of Vision

GPS Global Positioning System

MSN Mobile Sensor Network 


\section{Contents}

List of Figures $\quad 5$

1 Introduction $\quad 7$

1.1 Static Sensor Networks . . . . . . . . . . . . . . . . . . 7

1.2 Mobile Sensor Networks . . . . . . . . . . . . . . . . . . . . 8

1.3 Problem: Physical Obstacles . . . . . . . . . . . . . . . . . 10

1.4 The Model . . . . . . . . . . . . . . . . . . . . . . . . 11

1.5 Main Contributions . . . . . . . . . . . . . . . . . 13

1.6 Structure of the Thesis . . . . . . . . . . . . . . . . . 15

2 State of the Art $\quad 16$

2.1 Energy Restoration in Mobile Sensor Networks . . . . . . . . . . . . . . . . 16

2.1.1 Passive Approach to Energy Restoration . . . . . . . . . . . . . . 16

2.1.2 Proactive Approach to Energy Restoration . . . . . . . . . . . . 18

2.1.2.1 Creating the CDG . . . . . . . . . . . . 19

2.1.2.2 Migration Strategy . . . . . . . . . . . . . . . 21

2.1.2.3 Topology ....................... 23

2.1 .3 Conclusions . . . . . . . . . . . . . . . . . . 23

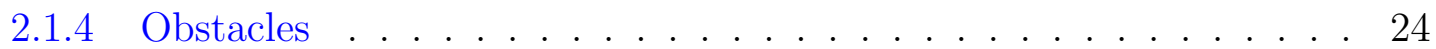

2.2 Control Strategies to Avoid Obstacles . . . . . . . . . . . . . . . 25

2.2.1 Bug Family . . . . . . . . . . . . . . . . . . 25

$2.2 .1 .1 \quad \operatorname{Bug} 1 \ldots \ldots \ldots \ldots$

2.2.1.2 $\operatorname{Bug} 2 \ldots \ldots \ldots \ldots \ldots$

2.2.1.3 Dist-Bug . . . . . . . . . . . . . . . 27

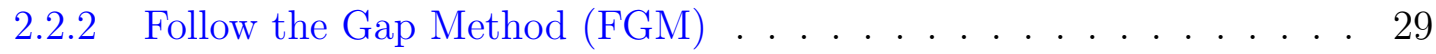

2.2 .3 Intelligent Bug Algorithm (IBA) . . . . . . . . . . . . . . . . . 29 
3 Proposed Modifications

3.1 Selection of the Obstacle Avoidance Strategy . . . . . . . . . . . . . . 32

3.2 Modifications to Energy Restoration Strategies . . . . . . . . . . . . . . 34

3.3 Using the Obstacle's Frame to Generate a Proactive Path . . . . . . . . . . 36

3.4 Improving the Obstacle's Frame . . . . . . . . . . . . . . . . . . . 38

3.5 Resolving the $\mathrm{U}$ and $\mathrm{H}$ Shape Problem . . . . . . . . . . . . . . . . . . 42

3.6 Polygonal Mazes . . . . . . . . . . . . . . . . . . . . . . . 45

3.7 Summary . . . . . . . . . . . . . . . . . . 46

4 Experimental Results $\quad 48$

4.1 Environment and Considerations . . . . . . . . . . . . . . . 48

4.1 .1 Performance Criteria . . . . . . . . . . . . . . . . . . . . 49

4.1 .2 Considerations About the Obstacles . . . . . . . . . . . . . 50

4.2 Passive vs Proactive Obstacle Avoidance . . . . . . . . . . . . . . 51

4.3 Adding Obstacles . . . . . . . . . . . . . . . . . . . 52

4.4 Sharing vs No Sharing the Obstacle's Frame . . . . . . . . . . . . . . 53

4.5 Travelling vs No Travelling the Graph . . . . . . . . . . . . . . . 56

4.6 One-Hop Runs vs Panic Runs . . . . . . . . . . . . . . . . . . . . 57

4.7 Looking for the Ideal Energy Restoration . . . . . . . . . . . . . 58

4.7.1 Increasing Sensor's Speed . . . . . . . . . . . . . . . . 59

4.7.2 Increasing Number of Sockets . . . . . . . . . . . . . . 59

4.7.3 Increasing the Value of the Recharge Threshold . . . . . . . . . . . 60

4.8 Proactive Strategies Comparison . . . . . . . . . . . . . . . . . . 61

5 Conclusions and Open Problems $\quad 63$

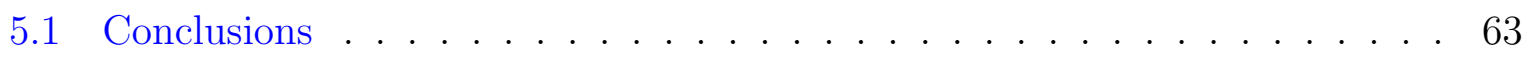

5.2 Open Problems . . . . . . . . . . . . . . . . . . 67

5.2 .1 Self-Deployment . . . . . . . . . . . . . . . . . 67

5.2 .2 Value of the Threshold $(\alpha) \ldots \ldots \ldots . \ldots . \ldots . \ldots$

5.2 .3 Communication . . . . . . . . . . . . . . . 70

5.2 .4 Topology . . . . . . . . . . . . . . . . . . 70

5.2.5 Complex Scenarios and Better Representations of the Obstacles . . 71 
Bibliography 


\section{List of Figures}

2.1 A Sensor's Life Cycle. . . . . . . . . . . . . . . . . . . . . . 19

2.2 Creation of the Compass Direct Graph (CDG) . . . . . . . . . . . . 20

2.3 Compass Direct Gabriel Graph. . . . . . . . . . . . . . . . . . . . 24

2.4 Bug 1 Algorithm. . . . . . . . . . . . . . . . 27

2.5 Bug 2 Algorithm. . . . . . . . . . . . . . . . . 28

2.6 Dist-Bug Algorithm. . . . . . . . . . . . . . . . . . 28

2.7 IBA Algorithm. . . . . . . . . . . . . . . . . . . . 30

3.1 Discovering the Obstacle while Recharging. . . . . . . . . . . . . . . . 35

3.2 Discovering the Obstacle while Swapping Positions. . . . . . . . . . . . . 35

3.3 Discovering the Obstacle while Recharging. . . . . . . . . . . . . . 37

3.4 Using the Obstacle's Frame to Generate a Safe Path. . . . . . . . . . . . . 37

3.5 Improving the Obstacle's Frame Accuracy. . . . . . . . . . . . . . . . . . . 38

3.6 Using the New Obstacle's Frame Information. . . . . . . . . . . . . . . . 39

3.7 Discovering the Obstacles. . . . . . . . . . . . . . . . . . . 40

3.8 Improving the Known Obstacle's Frame. . . . . . . . . . . . . . . . . . . . 40

3.9 Using the Known Obstacle's Frame. . . . . . . . . . . . . . . . . . . 41

3.10 Generating Short and Safe Paths. . . . . . . . . . . . . 41

3.11 Dead Scenario with U-Shaped Obstacle. . . . . . . . . . . . . . . . . . 42

3.12 Avoiding the U-Shaped Obstacle. . . . . . . . . . . . . . . . 43

3.13 Using an Obstacle's Frame to Generate a Short Path. . . . . . . . . . . . . 44

3.14 Flowchart of the IBA Modified Algorithm. . . . . . . . . . . . . . . 44

3.15 Worst-Case Scenario for a Sensor Inside a Polygonal Maze. . . . . . . . . . 46

3.16 Improving the Path Between Sensors. . . . . . . . . . . . . . . . . . . . 47

4.1 Different Shapes of Obstacles Used in the Simulations. . . . . . . . . . . . 50 
4.2 Passive vs Proactive Solutions in the Presence of Obstacles. . . . . . . . . 52

4.3 Adding Obstacles. . . . . . . . . . . . . . . . . . . . 53

4.4 Sharing vs No Sharing Obstacles Information. . . . . . . . . . . . . 54

4.5 Travelling vs No Travelling the Graph. . . . . . . . . . . . . . . . . . 56

4.6 Impact of the Number of Obstacles. . . . . . . . . . . . . . . . . . . 57

4.7 Panic Runs vs One-Hop Runs. . . . . . . . . . . . . . . . . . . . . 58

4.8 Speed of the Sensor. . . . . . . . . . . . . . . . . . . . . . . . . 59

4.9 Impact of Increasing the Number of Sockets. . . . . . . . . . . . . . 60

4.10 Impact of Increasing the Value of the Threshold $(\alpha) \ldots \ldots \ldots 1$

4.11 Proactive Strategies Comparison. . . . . . . . . . . . . . . . . 62

5.1 Worst-Case Scenario. . . . . . . . . . . . . . . . . 66

5.2 Model of a Sensor . . . . . . . . . . . . . . . . . . . . . . . 67

5.3 Self-Deployment Proposed. . . . . . . . . . . . . . . . . . . . 68 


\section{Chapter 1}

\section{Introduction}

Nowadays sensor networks are being used almost everywhere. Some of their most common applications in science and industry fields are in monitoring health care, monitoring environmental conditions, sensing forest fire, detection of natural disasters, energy saving, smart grids, and smart cities solutions (e.g., [19, 21, 22]). All these sensor networks have several main goals like having accurate sensing or an acceptable coverage, but one of the most important aspirations of any sensor network is to maximize sensor lifetime while performing the main task which they are meant to do with a high level of effectiveness. Every transmission, reception, movement, and operation performed by the sensors consumes energy. Without a solution for recharging or replacing batteries, the sensors' batteries will be depleted. If the batteries run out, then the loss of coverage will happen and the network will fail at some point. There are several approaches to solving this problem, depending on the type of network. Sensor networks can be classified as static or mobile, depending on whether the sensors can move or not.

\subsection{Static Sensor Networks}

Some approaches to energy restoration in static networks are gaining strength due to new technologies. These approaches are the ones based on extracting energy from the environment [9, 14]. For example, in [9] sensors have solar panels to recharge their batteries by themselves. Alternative approaches include special entities such as mobile actuators, recharge robots, or service stations to recharge, repair, and maintain the sensors (e.g., [30]). In cases like [40], the actuator or robot moves to the sensors and 
recharges their batteries. In [30], for example, the actuator is the one that carries a solar panel and recharges the sensor's battery with the energy saved by its solar panel. This strategy is completely reactive because the sensors are only recharged when their batteries are depleted.

All energy restoration strategies for static sensors can be grouped into two categories: cluster-based approaches (e.g., [8]) or mobility-based approaches (e.g., [40]). Almost all the solutions involve some kind of clustering or partitioning of the network with the special entities called cluster heads. The special entities must get information about the energy distribution of the sensors in the cluster in order to decide when to intervene. In these strategies, sensors play a passive role while the recharge robots or service stations play an active role [25].

\section{$1.2 \quad$ Mobile Sensor Networks}

Sensor mobility provides great value to networks because it allows the system to perform tasks that are impossible in static sensor networks. Mobile sensors can perform new tasks such as aerial and maritime unmanned applications (e.g., [6, 20,37, 43]), in addition to self-deployment (e.g., [24,31]). Excellent examples of the new tasks that mobile sensors can perform are tasks done by drones. In recent years, the use of drones to different sensing activities has increased. For example in [20], drones are used for forest monitoring while in [2] drones are used to assist in rescue service during natural disasters.

Mobile capability also allows sensors to solve the problem of energy restoration by themselves. Sensors can move towards recharge facilities deployed over the sensing area. In this way, they can play an active role in their energy management while the recharge stations play a passive one.

In this active role, the mobile sensors are responsible for managing their own energy levels. They also must be able to develop strategies to recharge their batteries in order to guarantee continuous operation. The idea is that sensors can extend their operating time beyond an initial recharge, by recharging themselves at service stations. Sensors 
also need to coordinate the use of service stations and decide when it is time to recharge based on their energy and some fixed threshold [38]. There are several proposals to solve the problem of energy restoration in networks where sensors are able to harvesting energy from the environment and do not require a recharge station (e.g., [5, 29, 42]). However, this research is focused in other type of networks where sensors have to travel to the recharge stations in order to recharge their batteries.

How to achieve continuous operation by refueling or recharging mobile entities is a well-known problem in the robotics field known as Frugal Feeding Problem (FFP) [17]. The idea behind FFP is to find energy-efficient routes for a mobile service entity called "tanker", to rendezvous with every member of a team of mobile robots. Depending on where the feeding takes place there are different variants of the FFP. There is a variant of the FFP called "tanker absorbed" where feeding takes place in a tanker's predefined location. The "tanker absorbed" approach to the FFP can be transformed into the problem of energy restoration in mobile sensor networks, where the sensors are the robots and the tanker is the recharge station. The main goal of this version of the FFP is that robots should reach the rendezvous location without a complete loss of energy during the process.

There are some necessary conditions for the sensors to be able to recharge themselves that must be fulfilled regardless of the scenario. In order to recharge by themselves, sensors must be able to locate the recharge station and be able to move toward it. They must also be able to manage their energy levels and detect when it is time to recharge based on some threshold. Sensors should have enough battery energy to reach the recharge station. There must be a mechanism for transferring the energy between the recharge station and the sensors. Energy can be transferred thru a physical connection by plug-in a recharge connector, or it can be done wireless if the sensor is inside the recharge area of the station (e.g., $[4,40])$. In our scenarios, it was assumed that the service station must have docks, ports, or sockets where the energy transfer occurs thru a physical connection.

The "ideal energy restoration strategy" is one that can guarantee a continuous operation of the network without any loss of sensors. But real life is messy, and achieving the ideal 
strategy is unlikely, or very costly. In common energy restoration strategies, the goal is to find a strategy where sensor losses are limited in scope and time. The goal is to reach a state where no further sensor losses will occur (state of equilibrium), with the minimum number of losses.

In $[25,35]$, new energy restoration strategies that reach the state of equilibrium were proposed. The results of a passive approach were compared to the results of a proactive approach to restoring the energy. In the passive strategy, the sensors managed their own battery levels at periodic intervals. Then when their batteries reached a critical level, the sensors tried to travel to the recharge station. By contrast, with the proactive strategy, sensors tried to move before their batteries reached a critical level. The idea was that sensors with less energy would try to get closer to their service stations by swapping positions with sensors with higher battery levels. Then when sensors with lower battery levels reached the critical level, they would be closer to the recharge station. The strategies described in $[25,35]$ yield competitive results. In chapter 2, section 2.1 these strategies are described.

The results achieved in [35] are based on ideal scenarios where there are no obstacles. But in real life, some communication and physical obstacles can interfere with the performance of the algorithms. As a next step to get closer to real life scenarios and following the recommendation made in [25], it was decided in the present thesis to enrich the scenarios by adding physical obstacles.

\subsection{Problem: Physical Obstacles}

Obstacles can be classified as physical obstacles and/or communication obstacles. Physical obstacles prevent the deployment and movement of sensors in their area, while communication obstacles interfere and interrupt the wireless communication between sensors. In the context of restoring energy in mobile sensor networks, physical obstacles acquire more relevance because sensors are moving over the field, and in some scenarios where the physical obstacles are present sensors will have to deal with them. 
Adding physical obstacles to the scenarios means that mobile sensors must be able to avoid the obstacles efficiently and be able to reach a target position without collisions. Regardless of the number and shape of the obstacles, sensors must avoid them based only on local information. The problem of obstacle avoidance in mobile robots has been one of the most analyzed and discussed topics in the field of robotics. In chapter 2, section 2.2, different control strategies for obstacle avoidance are analyzed.

In the specific case of having obstacles in mobile sensor network scenarios, different research have been carried out examining problems such as: how to deploy sensors in environments that have physical obstacles $[23,24]$, how to route packages efficiently in the presence of obstacles [7,10], and how to guarantee communication in topologies that contain obstacles [11]. However, how physical obstacles impact the performance of the energy restoration strategies in mobile sensor networks has not been analyzed until now, to the best of our knowledge.

This problem is the main focus of the present research. Also explored is the question of finding an efficient energy restoration strategy that can be applied in scenarios where physical obstacles are present. The energy restoration strategy found should achieve competitive results, therefore, the obstacle avoidance technique used by mobile sensors has to be efficient. Finally, the question of whether the ideal energy restoration strategy can be achieved in the presence of obstacles, and under what conditions, is examined.

\subsection{The Model}

The model used to analyze the problem of how to restore the energy of mobile sensor networks in the presence of physical obstacles is composed of three main components. The first is a set of $N$ mobile sensors $S=\left\{S_{1}, S_{2}, \ldots S_{N}\right\}$, already deployed over the field. The second component is a recharge facility $F$ that will have a fixed number of sockets or recharging ports. The last component is a set of $K$ physical obstacles $O=\left\{O_{1}, O_{2}, \ldots O_{K}\right\}$.

Sensors are endowed with motorial capabilities. They can move in any direction while they have enough energy to do it. All sensors will move at the same speed and when 
they are avoiding an obstacle they will always move in the same direction (i.e., clockwise).

Sensors will be deployed using any self-deployment algorithm (e.g., [23, 24]), and considering the obstacles' position on the field. Sensors can not be deployed over the area covered by an obstacle. It is assumed that the positions of the sensors on the field are those desired for the assigned sensing task. If a sensor moves from its position to the service station or to other sensor's position a sensing hole is created. A sensor knows its own position via a location equipment like a Global Positioning System (GPS). A sensor also knows the fixed position of its service facility. Sensors do not have any information about the obstacles.

It is assumed that each sensor is able to perform local computations and its memory is limited. They are equipped with a distance sonar or a touch detector that can determine if an obstacle is reached. Sensors can communicate with other sensors and the recharge station within their transmission range " $R$ ".

The deployment of the recharge station can be done using some clustering algorithms like $[8,39]$, but always guaranteeing that the station is not deployed inside a maze (one or multiple entrance maze). To avoid these scenarios, there must be at least one straight path from the recharge station to some point on the rectangular frame that contains all the obstacles. Otherwise, the recharge station can be deployed anywhere over the field but ensuring that it is not deployed over the area of an obstacle. The recharge station does not need to know any information about the sensors.

The communication is asynchronous, so there is no need for a global clock or entity to coordinate the communications. There is full reliability in the communication between the sensors. The communication environment is error free so it is not necessary to retransmit messages. Sensors and recharge facilities can receive several messages at the same time without collision.

Physical obstacles are located over the field without overlapping with any other component of the system (sensors, obstacles, and recharge station). No obstacle is too big to be avoided by a sensor with a full battery. It is considered that obstacles can 
have any circular or polygonal shape. The obstacle must be contained in a circle where the size of the radius is equal to the half of the transmission range $(r=R / 2)$. With this assumption, sensors can determinate how much time they will wait until receiving a response after sending a request, or receiving the information gathered after finishing a movement operation by the other sensor involved. For example, if while swapping positions one of the sensor involved in the operation dies in a position out of the transmission range of the other sensor, the surviving sensor after completing the swap, will wait a predefined time based on how much time could take to avoid an obstacle contained in an circle of radio $=R / 2$.

\subsection{Main Contributions}

The first contribution of this thesis is the integration of some concepts and algorithms from the obstacles avoidance techniques in the robotics field, into the energy restoration strategies in mobile sensor networks.

One major contribution of this thesis is the confirmation of the negative impact of adding physical obstacles to scenarios where the energy restoration strategies are applied. In response, this thesis proposes a new efficient proactive energy restoration strategy for mobile sensor networks (based on the proactive strategy described in [35]) that allows sensors to avoid obstacles efficiently whether the physical obstacles are polygonal shapes (including U- and H-shaped obstacles), circles, or polygonal mazes. In the specific case of polygonal mazes, if a sensor is deployed inside the maze, it will not survive, but it will avoid that other sensors get trapped inside the maze.

To avoid obstacles efficiently, the Intelligent Bug Algorithm (IBA) was used, but with some modifications. One of the modifications introduced is the practice of storing the four most extreme values of each obstacle discovered, and creating with these values, rectangular frames that represent the obstacles. The frames are used to improve the paths between sensors and the paths between sensors and the recharge station. With the frame information, sensors avoid unnecessary exploration. Another modification proposed in this thesis regarding the obstacle avoidance technique is to count the turns 
sensors make while avoiding the obstacles. With the information of the frames and the turn count, sensors are able to avoid U- and H-shaped obstacles based only on local information. Sensor are also able to solve polygonal mazes while the target position is not inside the maze.

It is proposed that sensors share the obstacles' frame information. The shared information is used by the sensors to avoid the obstacles efficiently, avoid unnecessary exploration, and generate safe paths. Energy restoration strategies in which this proposal has a positive benefit (proactive), and strategies in which it does not (passive), are discussed.

It is found that both energy restoration strategies, passive and proactive reach the "state of equilibrium" where some sensors survive, even in the presence of obstacles. It is shown in the simulation results that the proactive strategy has a much better performance than the passive one in presence of physical obstacles.

It is analyzed that the "state of perfect equilibrium" where there are no loss of sensors (ideal energy restoration) can be reached, but the amount and type of resources needed to achieve that state in the presence of obstacle are different that the amount and type of resources needed in [35], which had no obstacles. Besides increasing the number of sockets of the recharge station, reaching the state of perfect equilibrium in the presence of obstacles also require an adjustment to the threshold that defines when the battery needs to be recharged.

It was also found that in unknown environments, with a proactive strategy when sensors need to reach the recharge station, it is better to travel the graph created by the proactive energy restoration strategy proposed in $[25,35]$, than to travel directly to the station.

Finally, an analysis of the impact of different key indicators in the performance of the algorithms is done. The keys indicators are the number of sensors loss until reaching the equilibrium, the number of panic runs (recharges made from a distance greater than one-hop), and the number of one-hop runs. The analysis shows that the proactive strategy that shares the obstacles' information and travels the graph achieves the fewest sensor losses overall. 


\subsection{Structure of the Thesis}

Two important and different topics are analyzed and elaborated in this thesis as part of the state of the art: the energy restoration approaches described in $[25,35]$, and some of the most common strategies for obstacle avoidance. In chapter 2, these two topics will be described and related.

The chapter 3 is dedicated to describing our proposal to solve the problem of energy restoration in mobile sensor networks in the presence of physical obstacles. First, the choice of the Intelligent Bug Algorithm IBA as an obstacle avoidance strategy is explained. The proposal to keep and use obstacle information to produce a short path is introduced. This chapter also contains the modifications made to the IBA algorithm to be able to avoid obstacles in $\mathrm{U}$ and $\mathrm{H}$ shape and topologies that contain polygonal mazes. In addition, the way sensors use obstacle information and improve its accuracy is described. Finally, some improvements in the energy restoration strategies are described.

In chapter 4, some simulations are proposed to validate the effectiveness and the convergence of the strategies presented in chapter 3. Different key parameters like the number of sensor losses, the number of panics and one hop runs, and the number of sockets of the recharge station, are evaluated.

The conclusions drawn regarding the results achieved are presented in chapter 5 . In this chapter, some remarks and open problems are mentioned. 


\section{Chapter 2}

\section{State of the Art}

\subsection{Energy Restoration in Mobile Sensor Networks}

In $[25,35]$ novel strategies for energy restoration in mobile network sensors were introduced. In the context of the problem presented in [35], two main strategies were proposed. The first one was a passive strategy where sensors wait to recharge their batteries until the battery's energy reach the critical threshold. In the second strategy, sensors may move toward to the station before reaching the critical threshold, but they move by swapping position with their neighbours.

\subsubsection{Passive Approach to Energy Restoration}

The main idea behind the passive approach to energy restoration described in [35] is that sensors will have two basic battery states: BATTERY_OK and BATTERY_LOW. While sensors' batteries are OK they will remain at their position performing their main sensing tasks. When the battery level of a sensor falls below a predefined threshold $(\alpha)$, the sensor state will change to BATTERY_LOW. In this state, the sensor will try to ask the service station for an available socket to recharge. At this point, there are two different scenarios based on the ability of the sensor to communicate directly with the service station. If the recharge station is within the communication range of the sensor, the recharge request can be sent directly to the service station. Once the socket assignment is granted, the sensor can move to the recharge station. If the service station is out of the sensor's communication range, its request can be sent using some routing techniques 
(e.g., $[7,11])$, or the sensor can move toward the service station until the station gets in communication range or the sensor's battery dies.

A service station can receive several requests at the same time and these requests are stored in a queue and ranked based on different criteria. The ranking can be done based on several criteria such as: the request time, the battery level, the distance to the recharge station, or a combination of them. When a socket is available, the recharge station sends an RECHARGE_ACCEPT message with the assigned socket to the lower ranked sensor (e.g., closest sensor). After the recharge is done, the sensor sends a message to the station to let it know that the recharge is completed. Then, the sensor returns to its previous position in the deployment, and the station checks if there is any other RECHARGE_REQUEST message pending on the queue.

In [25], it was proved that the passive approach reaches the state of equilibrium, but with a high number of sensor losses in comparison with the proactive approach. However, as it is mentioned in [35] there are several factors which have great influence on the results. The factors are: number of sensors, distance from the sensors to the service station, number of recharge docks or sockets on the station, and recharge time of the batteries at the station. One way to achieve the equilibrium in this passive strategy with no losses (perfect equilibrium) is to increase the number of sockets at the recharge station until there is always one socket available for every recharge request. This result leads to the first question regarding the problems of adding obstacles. Do these conditions still apply to a scenario where there are obstacles on the fields? This problem is experientially analyzed in chapter 4 .

When sensors travel to the recharge station some temporary coverage holes are created. In order to fix this issue, in networks where a temporary loss of coverage is a problem, in [25] it was proposed to have spare sensors at the recharge station that can cover the sensing area of the sensors that are recharging their batteries. When a recharge request is received, and there is an available socket, the station will send one spare sensor to the position of the sensor that made the request. The spare sensor will cover that area, while the sensor that made the request is being recharged. After the sensor is recharged, it will become a spare sensor. For this solution to work, the number of spare sensors should be 
equal to the number of recharge sockets.

\subsubsection{Proactive Approach to Energy Restoration}

In the proactive approach proposed in [35], sensors do not wait until their battery levels are low to start moving toward the service station. The sensors try to catch what it is called "front seats". The front seats are sensor positions that are at one-hop distance to the service station. These positions are limited. Like in the passive approach, sensors have responsibility at their locations, so changing positions has to be an agreement between two sensors in order to avoid coverage holes. The sensor with lower battery level will move to a position closer to a station, and the other sensor that accepted the swap will then cover the original position of the sensor with less energy. With this process of swapping positions, sensors with less energy will get closer to the recharge station.

In the proactive strategy, sensors will have three states depending on their battery: BATTERY_OK, BATTERY_LOW, and BATTERY_CRITICAL. While in state BATTERY_OK, sensors will perform normal sensing activities. In this state, sensors will also accept swapping request from sensors with lower battery levels. In this state, if the battery level falls below a fixed threshold $(\beta)$, the sensor's state will change to BATTERY_LOW. In BATTERY_LOW state, sensors will start traveling toward a recharge station by swapping positions with sensors with higher energy levels. Finally, the last state is BATTERY_CRITICAL, in which sensors will try to recharge their batteries. Sensors fall in this critical state when their battery levels are lower than the threshold $(\alpha)$. While a sensor is in this state, it will try to communicate with the service station to demand an available socket to recharge its battery. When the socket is granted the sensor will travel to the service station. Figure 2.1 shows the different states during the life cycle of a sensor in the proactive strategy proposed in $[25,35]$

The most important part of the cycle described in Figure 2.1 is the migration behaviour. The sensors main goal in the proactive strategy is to reach the service station quickly and efficiently while migrating. This migration will be done based only on local information. 


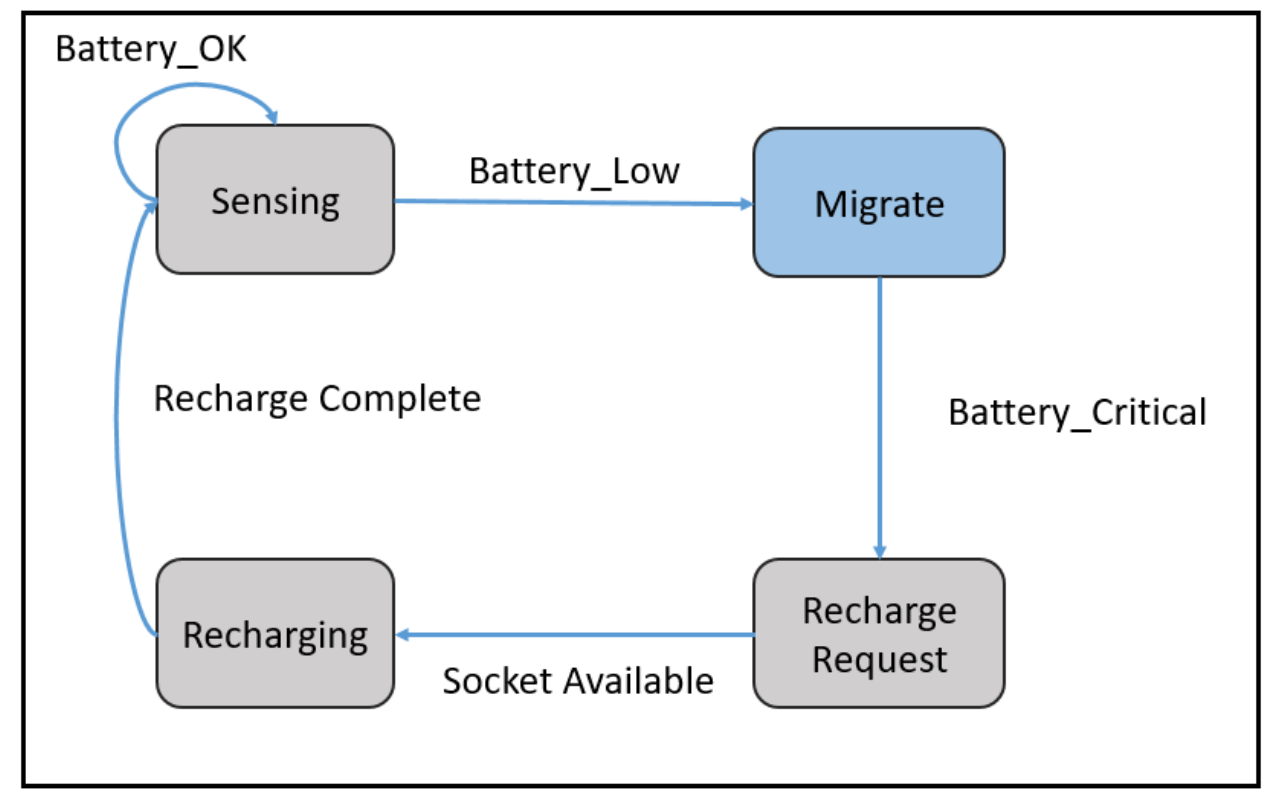

Figure 2.1: A Sensor's Life Cycle.

To achieve the migration [35] proposed to explore energy-aware routes that lead sensors to the service station. The idea is to create a graph where the sensors are the nodes and the recharge station is the root. The positions of the sensors are fixed, while the IDs are the ones that change when two nodes in the graph swap positions. In this graph, a sensor (node) will try to get closer to the recharge station (root) by swapping position with its parents. Then, the main idea is to use known position based routing strategies to reach the root efficiently (e.g., $[15,26]$ ). In common position base routing strategies nodes usually send messages to the root efficiently by minimizing the distance. But, in this case, instead of sending messages, the sensors will send themselves to the station's location by swapping position. The problem of coordinating the recharging of the sensors was converted into the problem of finding energy-aware routes in a logical Compass Direct Graph (CDG). In [35], a CDG was built over the original sensors' topology.

\subsubsection{Creating the CDG}

In [35], it is assumed that sensors are already deployed using some deployment technique like the ones described in $[27,32]$. Sensors have fixed positions depending on the network main task. Figure 2.2 shows an example of the creation of the CDG described in [35]. In this example, the creation of the CDG is described using three sensors $A, B, C$ and 
a recharge station $S$. It was assumed that all sensors have at least the minimum levels of energy to construct the CDG. Then, for sensor $B$ being neighbour of sensor $A$ in the CDG the following three criteria must be met:

1. Range. Sensors must be in communication range. For sensor $B$ being neighbour of $A$, the distance between them should be less than or equal to the transmission range. $d(A, B) \leq R$.

2. Proximity. The neighbour $B$ must be closer to the station. $d(A, S)>d(B, S)$ and $d(A, B)<d(A, S)$.

3. Directionality. Neighbour $B$ must be in the direction of the station. To ensure this, $B_{p}$ projection must intersects with line $A S$.

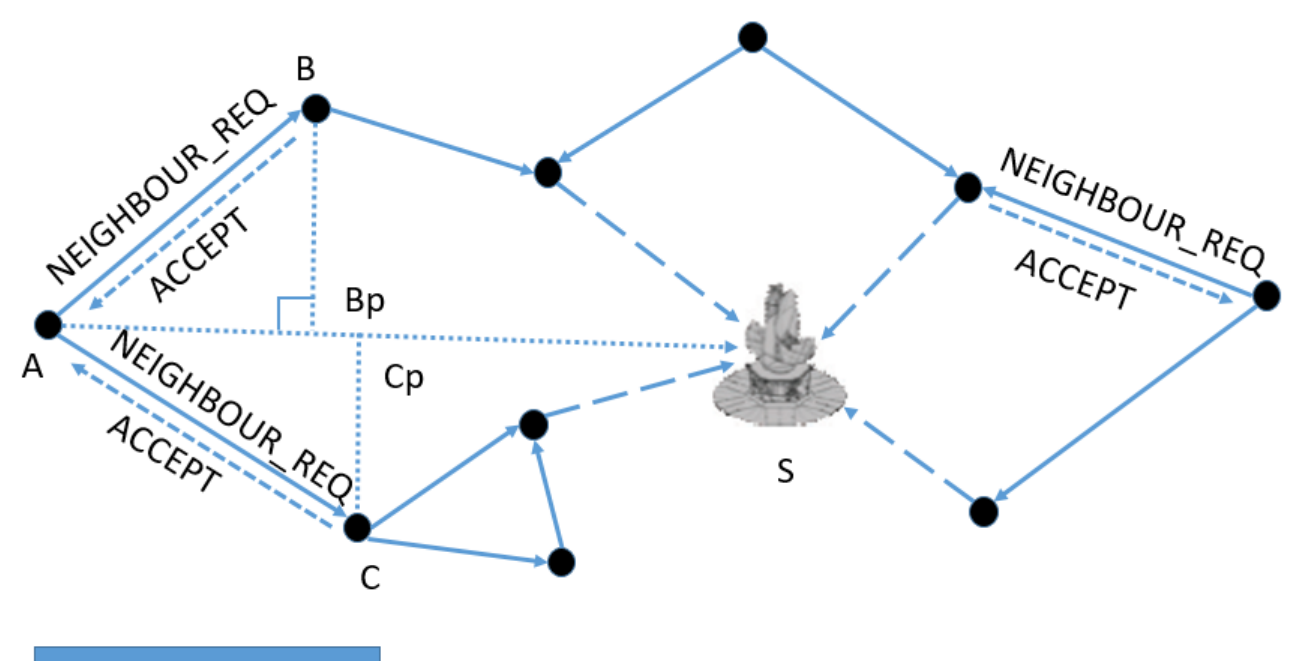

R: Transmission Range

Figure 2.2: Creation of the Compass Direct Graph (CDG).

If the conditions are met, then the sensor that received a NEIGHBOUR_REQUEST replies with a NEIGHBOUR_ACCEPT message. Otherwise, it sends a NEIGHBOUR_DENY message. Then, all the neighbours are ranked using a function to minimize the distance to the station. After this step, all sensors will have two routing tables. For example, sensor $A$ will have one table that contains all the sensors ( $B$ and $C$ ) that accepted the NEIGHBOUR_REQUEST message sent by $A$ (these sensors are added to its list of neighbours). The other table will have all the sensors that sensor $A$ accepted their 
NEIGHBOUR_REQUEST (children). After the creation of the CDG, the routing tables contain the position of the children and neighbour sensors, and the Euclidean Distance to them.

\subsubsection{Migration Strategy}

After the creation of the CDG sensors start the second phase. In this phase, a sensor with battery in BATTERY_OK state will perform the main sensing task. A sensor with battery level bellow the first threshold $(\beta)$ will start the migration and its state will be set to BATTERY_LOW. It will try to get closer to the service station by swapping positions with its neighbours. The sensor will send a SWAP_REQUEST message to its lowest ranked neighbour.

If the battery level of the neighbour that receives the message is higher, it will send a response message accepting the SWAP_REQUEST message, and it will travel to its children's position. During the travel in the swapping process, both sensors will not accept any other request. When the swapping is done, sensors will send a SWAP_COMPLETE message that will be used to update the information of their routing table. The neighbours and children of the sensors involved in the swapping use the information of the SWAP_COMPLETE message to update their tables too. If it is necessary to demand the sensors involved in the swapping to update its own table, the neighbours will send NEIGHBOUR_UPDATE message, and children will send a PARENT_UPDATE message. This happens because several swapping can happen at the same time and the routing tables must be updated.

On the other hand, if the battery level of the lower ranked neighbour is lower than the one who made the swap request, the neighbour replies with a SWAP_DENY message. Then, the sensor will try to swap position with the second neighbour sensor in the ranking, and so on until all neighbours have been verified. If all its neighbours are busy, or have less energy than the sensor, it will wait until one of its neighbours send a SWAP_COMPLETE, or a RECHARGE_COMPLETE message letting it know that this neighbour is available. Otherwise, the sensor will continue waiting and eventually its 
battery level will reach the critical state.

The last state is when the sensor's battery level falls bellow the second threshold and then the battery state changes to BATTERY_CRITICAL. In this state, sensors will have the same behaviour than in the passive approach. They will try to get a recharge at the service station. They send a RECHARGE_REQUEST message and wait for the recharge socket assignment. Like in the passive approach, if the station is in the communication range of the sensor, the sensor will send the message directly to the recharge station. If the service station is not in range, the sensor will try to route the request using some routing algorithm like $[3,12,26]$, or in case that there is not communication, the sensor will start travelling to the station until the station is in transmission range.

Once the sensor recharge is completed, it will return to the previously occupied position before it went to recharge. When the sensor returns to its previous position, it needs to rediscover the IDs of the sensors in its routing tables. Then, it will send a RECHARGE_COMPLETE message to its children and neighbours. If the IDs of the children or neighbours are different than the ones that the sensor have in its routing tables, they will send it a message with the correct ID. The RECHARGE_COMPLETE message also serves to let its children sensors to know that the sensor is available to swap positions.

The routing tables of sensors are position based tables. For a sensor the positions on the field of its children and neighbours are statics, while the IDs of them are dynamics. For that reason, in order to be able to update the changes in the network, the SWAP_COMPLETE and RECHARGE_DONE messages are used as triggers. The sensor that receives these messages will update its tables and in the case that the sender is not updated, they will send back a message with the information necessary to fix the sender routing table. The message will be a PARENT_UPDATE, or a NEIGHBOUR_UPDATE message, depending on whether the sensor is a neighbour in the first case or a child in the second one.

Finally, one of the most important aspects of the migration process presented in [25], and in [35] is that sensors reach the station in a finite number of swapping and there is not a cycle in the path. 
The ideal system is the one that all sensors batteries reach the state of BATTERY_CRITICAL when they are in the front seats. This means that sensors in BATTERY_CRITICAL state will be always at one-hop distance to the service station. All the recharges done at distance one-hop were called "optimal runs", or "one-hop runs", while the recharges made from other positions were called "panic runs". The count of panic runs and one-hop runs was used as a measure of the quality of the solution in [35].

\subsubsection{Topology}

In $[25,35]$, different underlying topologies were tested in the migration strategy. To construct these topologies, sensors only used local information. The topologies tested were flexible, dynamic, self-correcting, and loop-free. In [35], it was proposed to explore topologies where neighbours selection was based on the concept of Gabriel Neighbours and Relative Neighbours. Two sensor $A, B$ are Gabriel Neighbours if its diameter circle does not contain any other sensor. Then, a Gabriel Graph is a graph where all Gabriel Neighbours are connected. For example, in Figure 2.3 it can be appreciated that sensor $A$ with coordinates $(X i, Y i)$ and $B$ with coordinates $(X j, Y j)$ are neighbours because the circle with center $((X i+X j) / 2,(Y i+Y j) / 2)$ and radio $r=$ distance $(A, B) / 2$ does not contain any other sensor. Relative Neighbour Graph is a variant of Gabriel Graph where two sensors are neighbours if the Lune between them does no contain any other sensor. The different experiments made in [35] showed that with Gabriel Graph Topology the energy restoration strategies achieved better results.

\subsubsection{Conclusions}

In $[25,35]$, the problem of coordinating the recharging of mobile sensors was reduced to the problem of finding optimal routes in a logical Compass Directed Graph (CDG).

Two approaches that reach the state of equilibrium using the local information only were presented. The approaches are dynamic and self-correcting, therefore, sensors can be added or deleted during the simulations. After several experiments made in [35], 


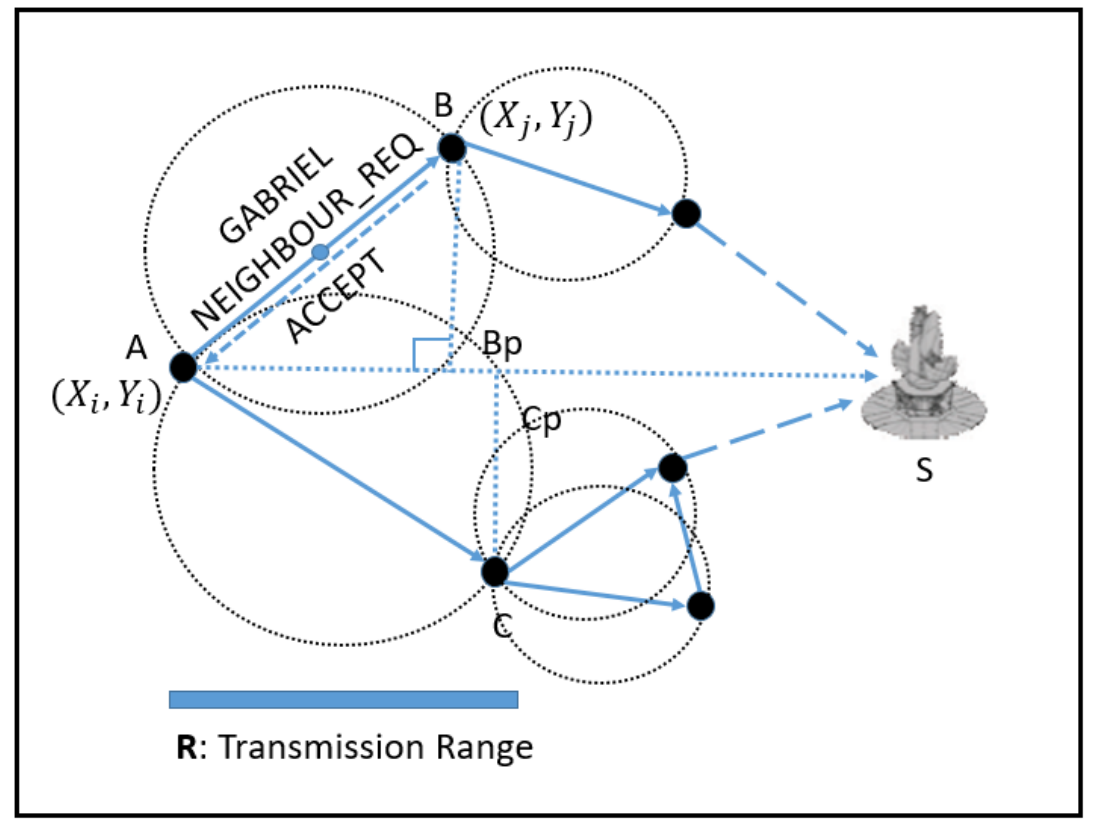

Figure 2.3: Compass Direct Gabriel Graph.

and [25], it was concluded that the proactive strategy achieved better results than the passive strategy in term of sensor losses until achieving the state of equilibrium.

In addition, it was analyzed the impact on the simulation results of different parameters like the number of recharge sockets, the transmission range, and new underlying topologies based on the different neighbours selection process. In the experiments made, it was found that the best improvement that can be done to the network in order to achieve the perfect equilibrium was to increase the number of recharge sockets at the station. It was also found that using Gabriel Graph as underlying topology, both strategies achieved better results. In [35], it was also validated that for a networks of 100 sensors the best results were archived with a transmission range of $100 \mathrm{~m}$, and $200 \mathrm{~m}$. One of the most important proposal made in [25] was to analyze the performance of the energy restoration strategies in scenarios where obstacles are present.

\subsubsection{Obstacles}

In this thesis, we focus on the impact that could have physical obstacles on the performance of the energy restoration strategies. Especially on the energy restoration strategies presented in [35] due to the competitive results that research obtained. Having 
physical obstacles on the scenarios mean that mobile sensors must have a mechanism to avoid collisions while swapping position, and move toward the recharge station. This mechanism to avoid obstacles must be efficient and produce the shortest possible path in the shortest possible time. Furthermore, it should use only local information. The problem of how to avoid obstacles efficiently has been deeply investigated in robotics.

\subsection{Control Strategies to Avoid Obstacles}

Recent technological advancements have made possible that mobile robots are being used almost everywhere. Robots must be able to interact with the environment, and performance some basic tasks as avoiding obstacles, path planning and navigation. Obstacles avoidance have been since the beginning of the robotics one of the most important tasks. Autonomous robots must be able to reach the destination target without any collision. They also must be able to generate the shortest path from the source to the target using only sensorial information of the environment. Robot navigation algorithms can be classified into three categories depending on the knowledge about the environment: completely known, partially known and unknown environment. In scenarios where robots do not know anything about the environment, the obstacle avoidance strategy becomes the backbone of robot navigation. The robots need to plan a safe path to the target using only target location, and sensorial information about the surrounding environment. Some of the most common control strategies for avoiding obstacles without environment knowledge are the following.

\subsubsection{Bug Family}

Bug algorithms have been one of the first, and most commonly used strategies for path planning. They have been proved, and implemented in multiple occasions. These algortihm have been used in several applications, for example, a version of Bug Algorithm was used in the Mars-Rover for planetary exploration [16]. These type of algorithms assumed that the robot is a point that has perfect localization, and the sensors of the robot are precise [41]. Bug algorithms require minimum memory, 
and do not require high computation [44]. They are fundamental and complete algorithms with provable guarantee [45]. Robots can detect when the destination is not reachable and they can stop the moving task. In addition, they only used the current sensors information to decide the next action. These algorithms are not goal oriented. An important characteristic of these algorithms is that robots will always move in one direction when they reach an obstacle, (e.g., clockwise, or counter-clockwise).

The main concern about algorithms in the Bug family is that the obstacle avoidance techniques can sometimes take the robot far away from the target [45]. Moreover, bug algorithms do not consider other obstacles during the edges detection process. A robot that uses any of the Bug family algorithms always knows its position, and the position of the target.

Robots in the bug family algorithms have two states while moving: "move to goal", and "obstacle avoidance" [46]. When a robot is in "move to goal" state, it can move freely. When an obstacle is detected, the state changes to "obstacle avoidance" state. While robots are in the "obstacle avoidance" state, they will move around the obstacle until they find the leaving point. The leaving point is a point in the path created by the robot while it moves around the obstacle, in which the robot will stop moving around, and will start moving in the direction to the target. At this point, the state changes to "move to goal". The main difference between the distinct categories inside the Bug Family is how they select the leaving point, while they are in the "obstacle avoidance" state.

\subsubsection{Bug 1}

In this variant of the algorithm, when a robot found an obstacle, it starts moving around the obstacle following the edges until reaching the point from where it started. While the robot is moving around the obstacle, for each point it visits, it calculates the distance from that point to the target. The point with the minimum distance to the target will be the leaving point. Then, the robot from the initial contact position follows the edges once again until reaching the leaving point. From this point, it calculates a new path, but in a straight line ("move to goal" state). If more obstacles are found, the process is the same 
for each one [41,44]. Figure 2.4 shows the avoidance obstacle strategy proposed in Bug 1.

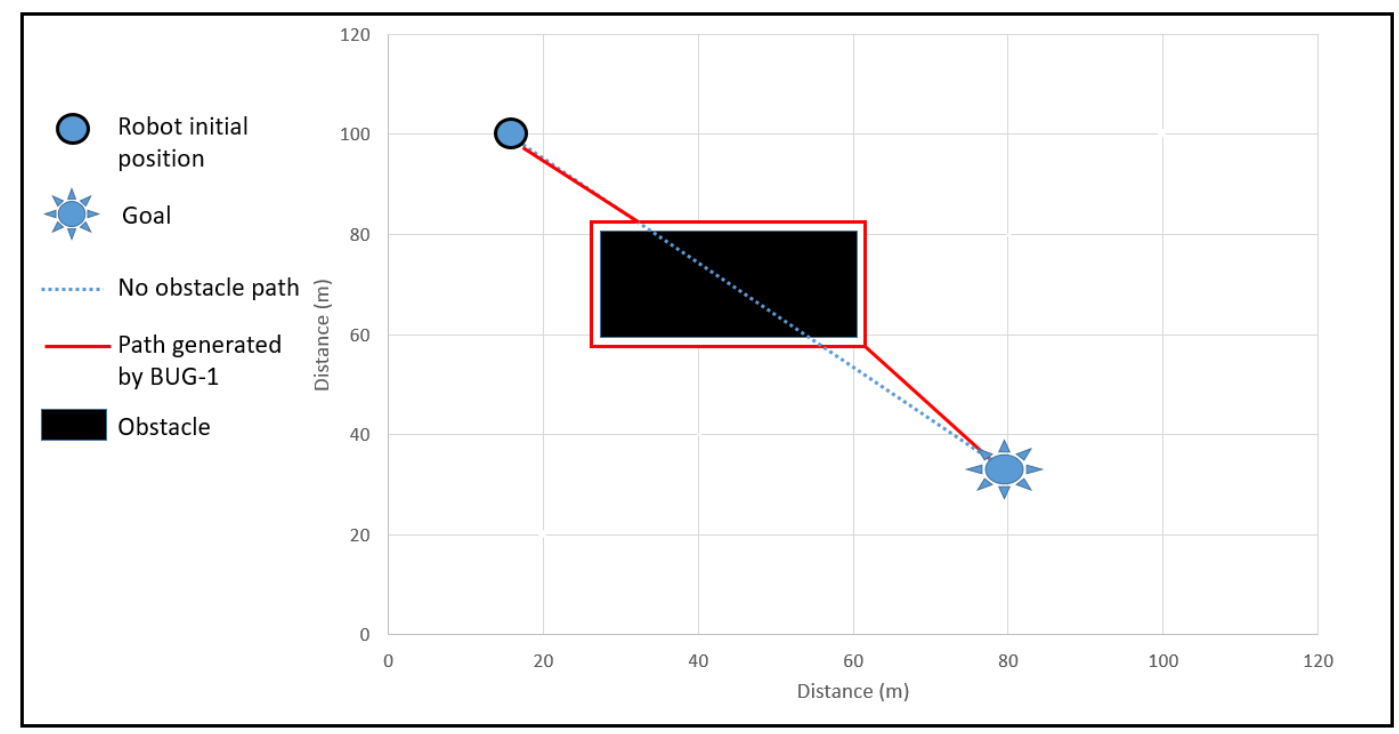

Figure 2.4: Bug 1 Algorithm.

\subsubsection{Bug 2}

This is an improvement of Bug 1. In Bug 2, the main idea is that the robot stores the slope $m$ from the straight line path from the source to the target. When a robot reaches an obstacle it starts moving around following the edges. While the robot is moving around the obstacle, and it has to decide where is the next position to move, the robot calculates the slope $m^{\prime}$ of the line from its current position to the target. When the robot reaches a point where $m^{\prime}=m$, then this point will be the leaving point, and the state changes to "move to goal". This strategy is better than Bug 1 because it allows the robot to reach the target faster than Bug 1 [45]. Figure 2.5 shows this obstacle avoidance strategy.

\subsubsection{Dist-Bug}

The Dist-Bug algorithm is the most efficient algorithm from this first known family of Bug Algorithms. In this algorithm the cost of the path is considered, while the robot is deciding the leaving point. This algorithm allows robots to reach the target faster than the previous versions. When a robot reaches an obstacle, it starts moving around the edges. For each decision step where the robot has to decide the next position to move around the obstacle, the robot calculated the distance from the current position to the goal, and the distance from the next position in the path that follows the edges to 


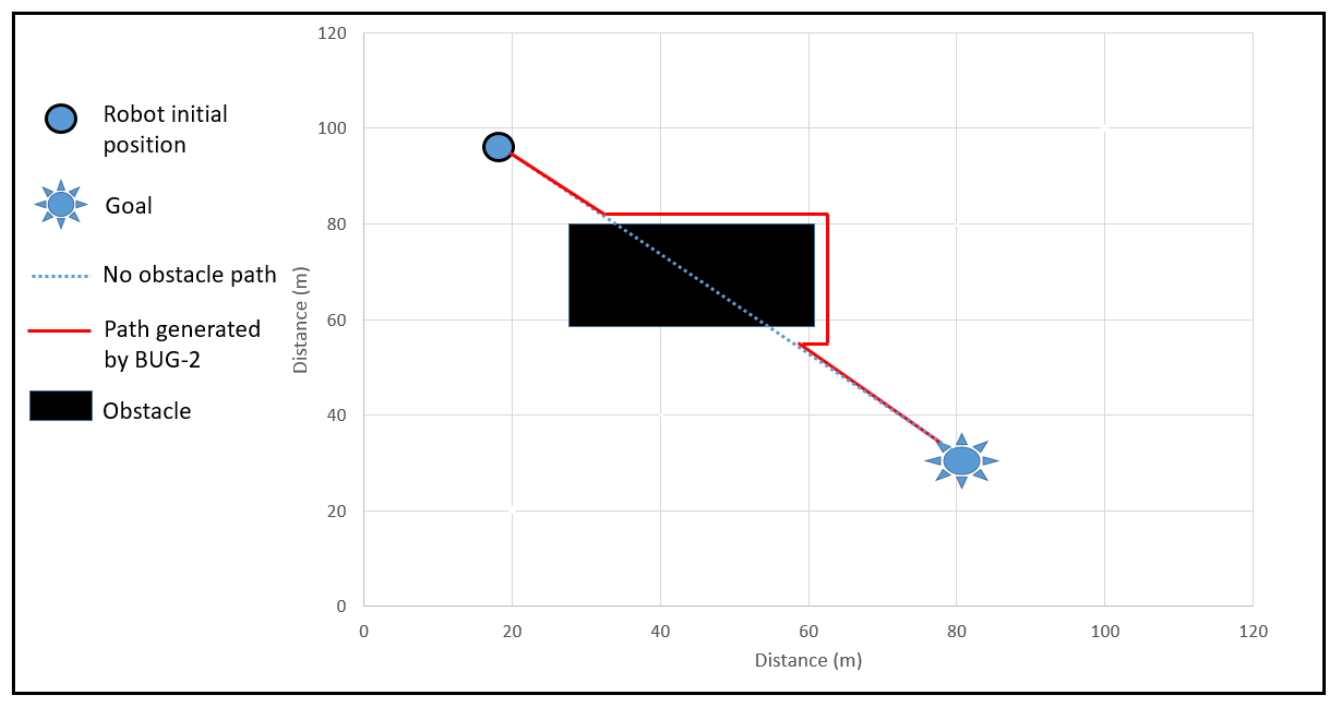

Figure 2.5: Bug 2 Algorithm.

the goal. In this case, the leaving point is selected when the distance between the next position around the obstacle and the goal position is greater than the distance between the current position and the goal. When the robot finds the leaving point it switches to "move to goal" state $[44,46]$. This algorithm is a greedy algorithm because it always selects the path that minimize the current distance. Figure 2.6 shows the performance of this strategy.

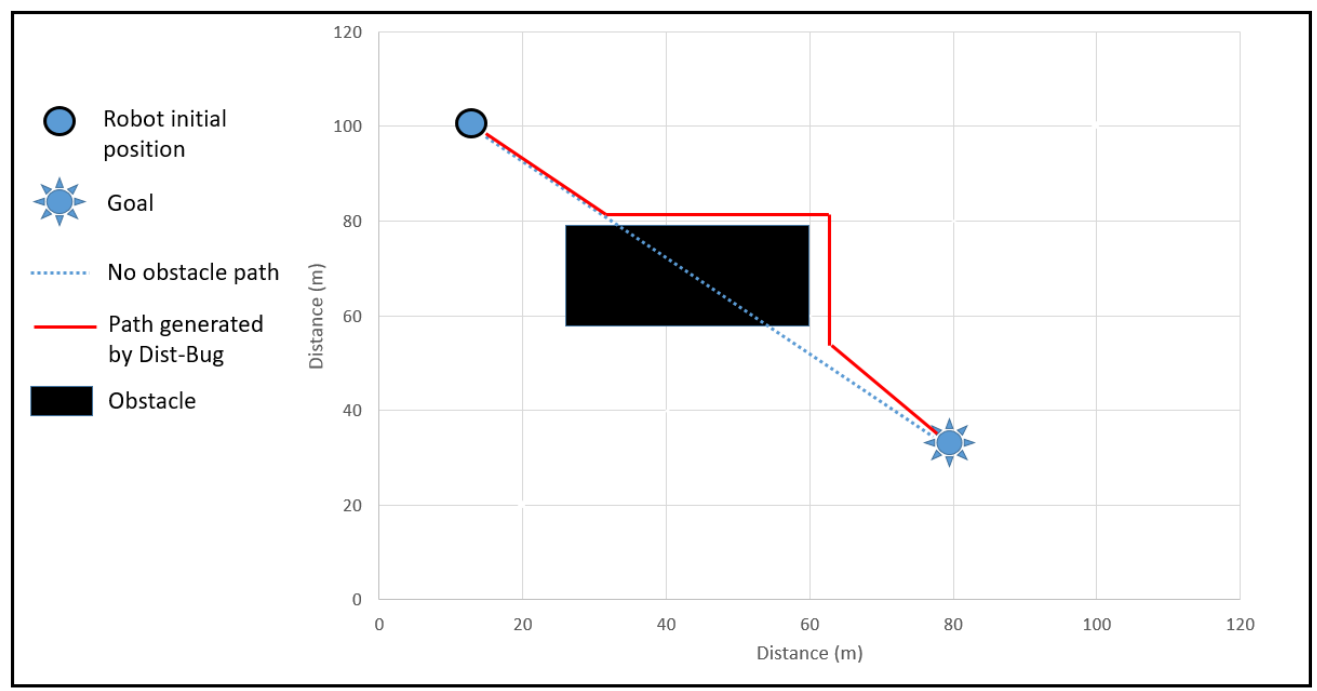

Figure 2.6: Dist-Bug Algorithm. 


\subsubsection{Follow the Gap Method (FGM)}

Follow the Gap Method (FGM) is another algorithm for obstacle avoidance presented in [28]. This algorithm introduces the concept of Field Of Vision (FOV). In this strategy, robots must have ultrasonic sensors, or an optical camera that generate the FOV. The idea of this algorithm is to avoid obstacles by finding the gap between them [44]. Robots will have a minimum gap threshold in which robots can move thru. Then, when obstacles are discovered by the FOV, the robots calculate the gap between the obstacles, and if it is greater than the threshold, the robot will follow the calculated gap angle. In case there is a single obstacle, the robots calculate the border angle that minimizes the distance to the target. The decision of where to move is made based on the current perspective, and the target location only. This strategy is more efficient than Bug Algorithms but requires more hardware, and memory resources. Besides, it has dead scenarios like obstacles in "U" and "H" shapes. FGM assumes that robots, and obstacles are circular.

\subsubsection{Intelligent Bug Algorithm (IBA)}

The Intelligent Bug Algorithm IBA was proposed in [45]. This algorithm for avoiding obstacles is similar to Bug Family algorithms but in this case, the strategy is goal oriented. In every step of this strategy, during the obstacle avoidance, the goal position is considered. This algorithm only uses local sensorial information, and the goal position to determine where to move. Like in the Bug Family Algorithms, IBA has two states: "move to goal", and "obstacle avoidance". Robots will calculate the straight path from the source to the goal, and will follow it until an obstacle is reached. When a robot discovers an obstacle, the robot's state will change to "obstacle avoidance". The robot will follow the edges of the obstacle until finding the leaving point. How IBA select the leaving point is the main difference with the Bug Family Algorithms. In this case, the leaving point will be selected based on the possibility of free path toward the goal position. This makes IBA algorithm goal oriented. Robots do not need to find a point with a minimum distance to the goal to leave the edges of the obstacle. When a robot finds a point in the obstacle edges where it can move free from that point to the target, that will be the leaving point. The robot's state will change to "move to goal", and it will try to generate a new straight path from the leaving point to the goal position. 
In [45], it was proved that the IBA algorithm generates a shorter and smoother trajectory than all the algorithms of Bug Family. The robot reaches the goal position in less time than other algorithms. IBA can be bidirectional if the robot uses FOV, and the sensor's configuration is modified. Like the Bug Family Algorithms, IBA has some limitations, like considering the robot as a single point with no dimensions. The decisions made in the IBA algorithm are based on current sensorial information of the surrounding environment, therefore, some noise in the information could impact the performance. This approach also has dead scenarios when the obstacles have "U", or "H" shapes. Figure 2.7 shows the performance of this algorithm.

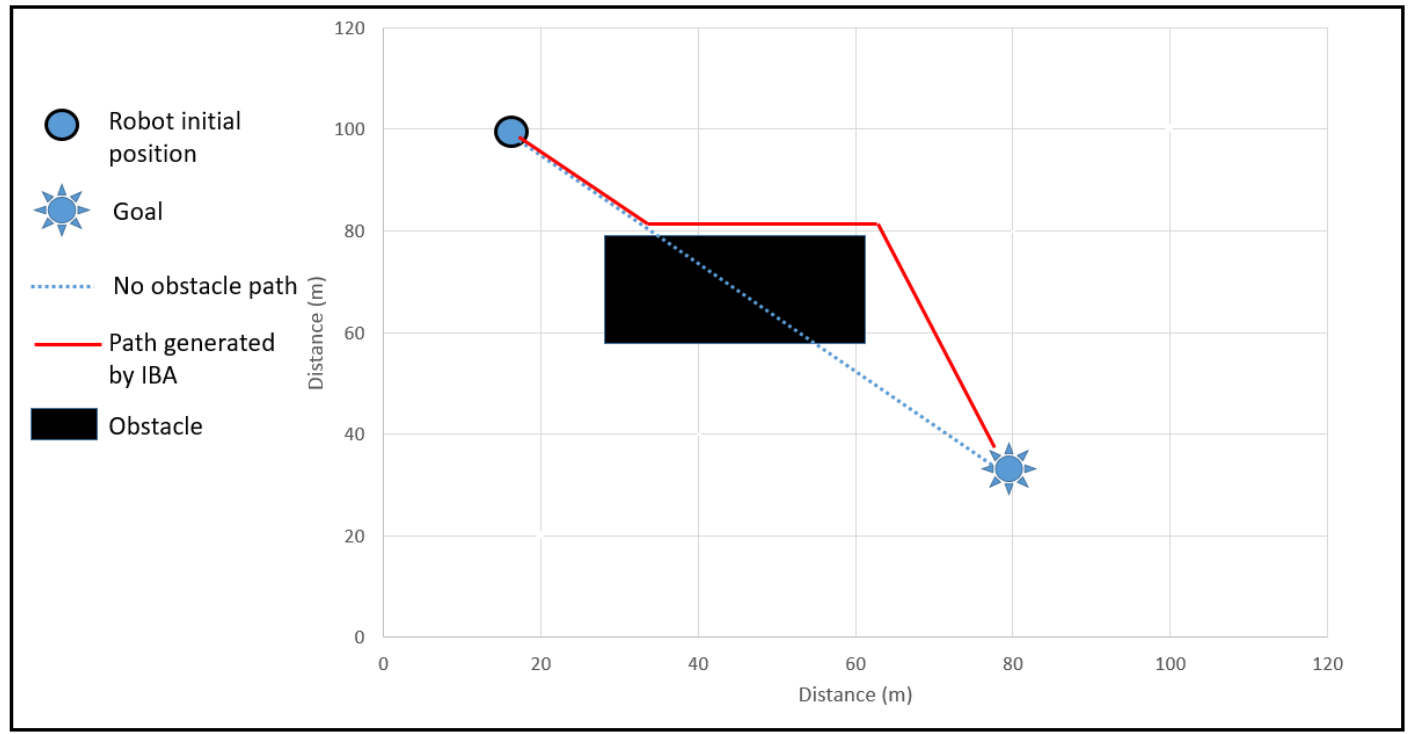

Figure 2.7: IBA Algorithm.

\subsubsection{Conclusions and Limitations of the Obstacle Avoidance Strategies}

All the obstacle avoidance strategies described before have been analyzed and compared in several papers like [18,44-46]. From the results obtained, it can be concluded that FGM, and IBA achieve the best results. FGM reaches the target position faster than the others but it requires more resources (ultrasonic sensors, camera). In contrast, IBA is the second best in terms of reaching the target while minimizing the distance, it is also 
easy to tune, and it does not require too many resources as FGM.

FGM, and IBA have important limitations. Both strategies are unable to avoid U- and H-shaped obstacles. FGM fails due to its local characteristics, while IBA fails due to its goal oriented characteristic. In addition, FGM considers that all the obstacles and the robot are circular. But, FGM takes the robot's dimensions as a variable to calculate if the robot can move thru the gap. On the other hand, IBA algorithm represents the robot as a point, therefore in scenarios where the robot dimension is important, some modifications have to be done.

Finally, it is important to remark that in our applications we are dealing with sensors, not robots. Robots can deal with more complex scenarios and can perform more complex activities than sensors. That is why in the context of our applications, the resources needed for the robot to avoid the obstacles are an important factor to consider when selecting the obstacle avoidance technique to be used. 


\section{Chapter 3}

\section{Proposed Modifications}

\subsection{Selection of the Obstacle Avoidance Strategy}

In chapter 2, different strategies for obstacle avoidance were described. It was mentioned that IBA, and FGM were the algorithms that achieved better results in terms of the shortest and smoothest trajectory. All the techniques described in chapter 2 can be easily modified to keep information about the avoided obstacles. Obstacle avoidance techniques can be classified as passive or proactive depending on whether robots keep and use information about the obstacles. This information can be used for path planning and collision avoidance.

In proactive obstacle avoidance techniques when a sensor avoids an obstacle it is not only important to do it efficiently, but it is also important to gather as much information as possible about the position and area of the obstacle without sacrificing too much energy in the process. This information can be used to improve the path, and could also be shared with other sensors to avoid the exploration of a known obstacle.

With the BUG-1 algorithm the real area and position of the obstacle can be discovered, but as it was shown in $[44,45]$, the cost in terms of time and energy for this is too high compared to other algorithms described in chapter 2. It is important to emphasize that in the energy restoration strategy while a sensor is moving to avoid an obstacle, it creates a temporary coverage hole in its area. The sensor must collect useful information about the position and area of the obstacle in the shortest possible time while moving to avoid 
it. It is also important to consider that the sensor's memory in our application is limited. Therefore, the key is to identify and store the most relevant and useful information about the obstacle that can improve the obstacle avoidance technique.

In the specific case of avoiding obstacles while restoring the sensor's energy using the strategy described in [35], it was decided that the IBA algorithm was the approach that best satisfies the needs of the problem. The reasons for this selection were: the good results that IBA achieves in terms of time and generating short paths, the small amount of resources and equipment that robots need to perform this obstacle avoidance technique, and the fact that the IBA algorithm has a variant that always moves in the same direction when reaching obstacles (i.e., clockwise or counter-clockwise). This variant does not need the sensor to be equipped with an optical camera or ultrasonic detectors. It works with a simple touch sensor, allowing the sensors to be simpler.

The characteristic of moving always in the same direction is useful in our context. It is true that moving in the same direction every time can lead to a long path but it is a small price to pay in order to be able to create a representation of the area and position of the obstacle. The balance between finding a short path and obtaining enough useful information about the obstacle is reached with the IBA algorithm. By using IBA to avoid obstacles in the energy restoration strategy presented in [35], sensors can estimate the obstacle's position and the area that it occupies. This estimation of the area and position of the obstacle can be done because every movement operation in the energy restoration technique described in [35] has its reverse operation, which enables the obstacles to be partially mapped.

In our applications, the operations that involve movements are the swap operation and the recharge operation. The round trip to the recharge facility is considered as a single movement operation regarding the issue of discovering the area and the position of the obstacle. Swapping is also considered as a single operation. In both, the swapping and the recharge operations, sensors will try to travel in a straight line to the destination. If there is an obstacle in the path this line will cut the obstacle in two and if the sensor moves always in the same direction when reaching an obstacle, it can be ensured that the two parts of the obstacle will be touched by the sensors. Consequently, two paths 
will be generated, one on each side of the obstacle. This exploration on both sides of the obstacle allows the sensor to select and keep the shortest path. In addition, both paths will create an envelop that contains the obstacle.

\subsection{Modifications to Energy Restoration Strategies}

For each face of the obstacle that a sensor touches, it will keep and update information about the obstacle. This information will be a representation of the 4 most extreme values touched by the sensor while avoiding the obstacle. The sensor keeps and updates only these four values per obstacle $(X \min , X \max , Y \min , Y \max )$. Every time the sensor moves around an obstacle it will update these values with the $X$ and $Y$ values of the point where the obstacle intersects with the straight line between the sensor's current position and its target.

Using only 4 values to represent each obstacle ensures that the memory of the sensor will not be overloaded with information about the obstacle. With these 4 values, sensors can generate the 4 corner points of the obstacle's frame

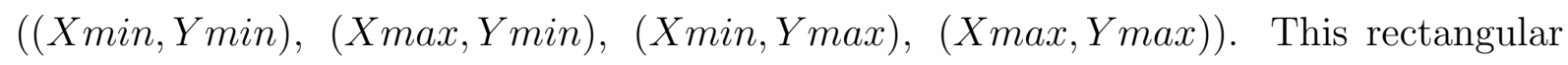
obstacle frame will represent the area and position of the discovered obstacle.

Figure 3.1 and Figure 3.2 show how to use the IBA to avoid obstacles while sensors perform the swapping operation and the recharge operation. The area and position of the obstacles can be estimated using the obstacle's frame.

Figure 3.1 shows how sensor $A$ discovers both sides of the obstacle cut by the line $A F$. When the sensor $A$ travels to the service facility, it avoids the obstacle efficiently using only local information. While travelling around the obstacle to the service facility, the sensor keeps information about the $X \min , X \max , Y \min , Y \max$. When the sensor returns to its original position, it updates the values that represent the obstacle's frame with the information about the other side of the obstacle. Once the sensor returns to its original position, it selects the shortest known path to the station between the two paths explored. The shortest known path to the recharge station is updated in the routing 


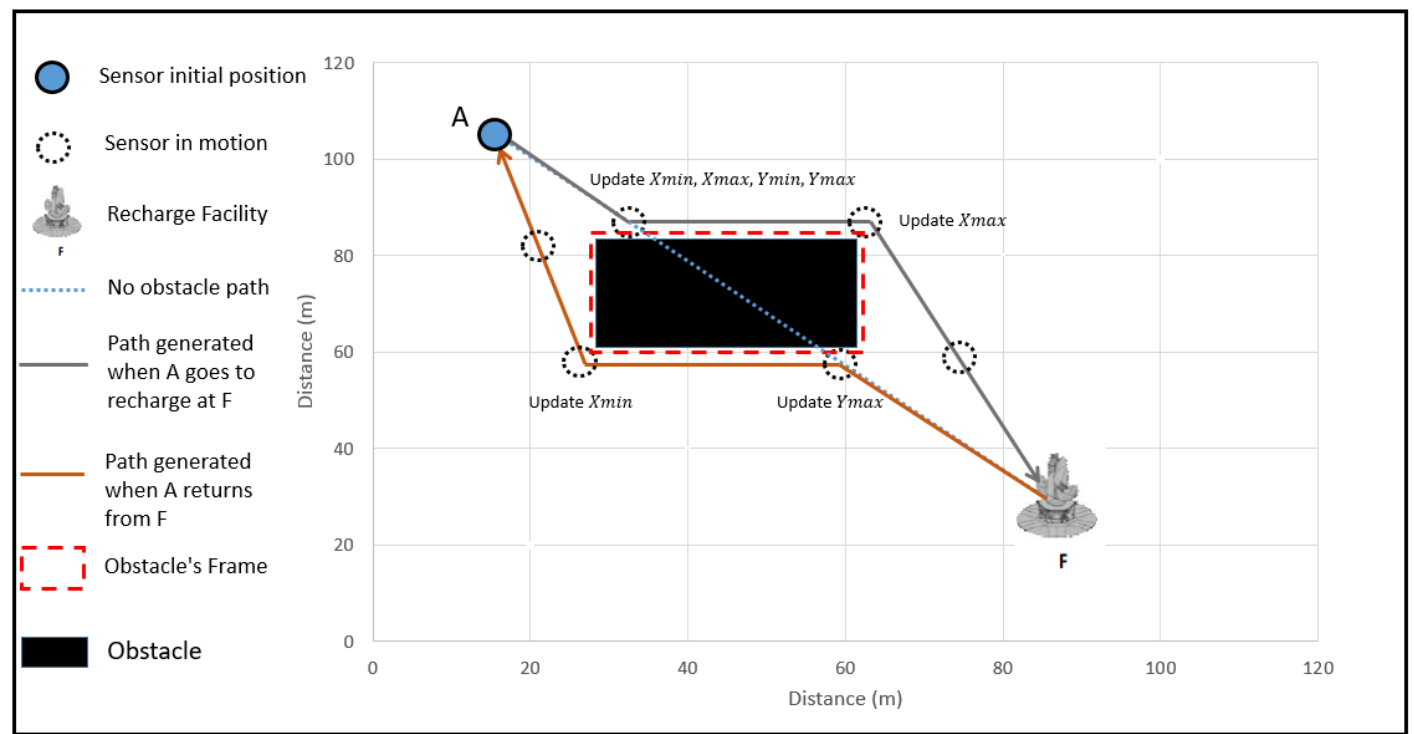

Figure 3.1: Discovering the Obstacle while Recharging.

table. In addition to the routing tables, the sensor will have a list of known frames. Each obstacle will be represented by a frame created with its most extreme known values. At the end of the recharge operation, the sensor will have updated the shortest known path to the station and will have enough information to create a rectangular frame that represent the obstacle found. This obstacle frame is used to improve the known path, and to avoid unnecessary exploration.

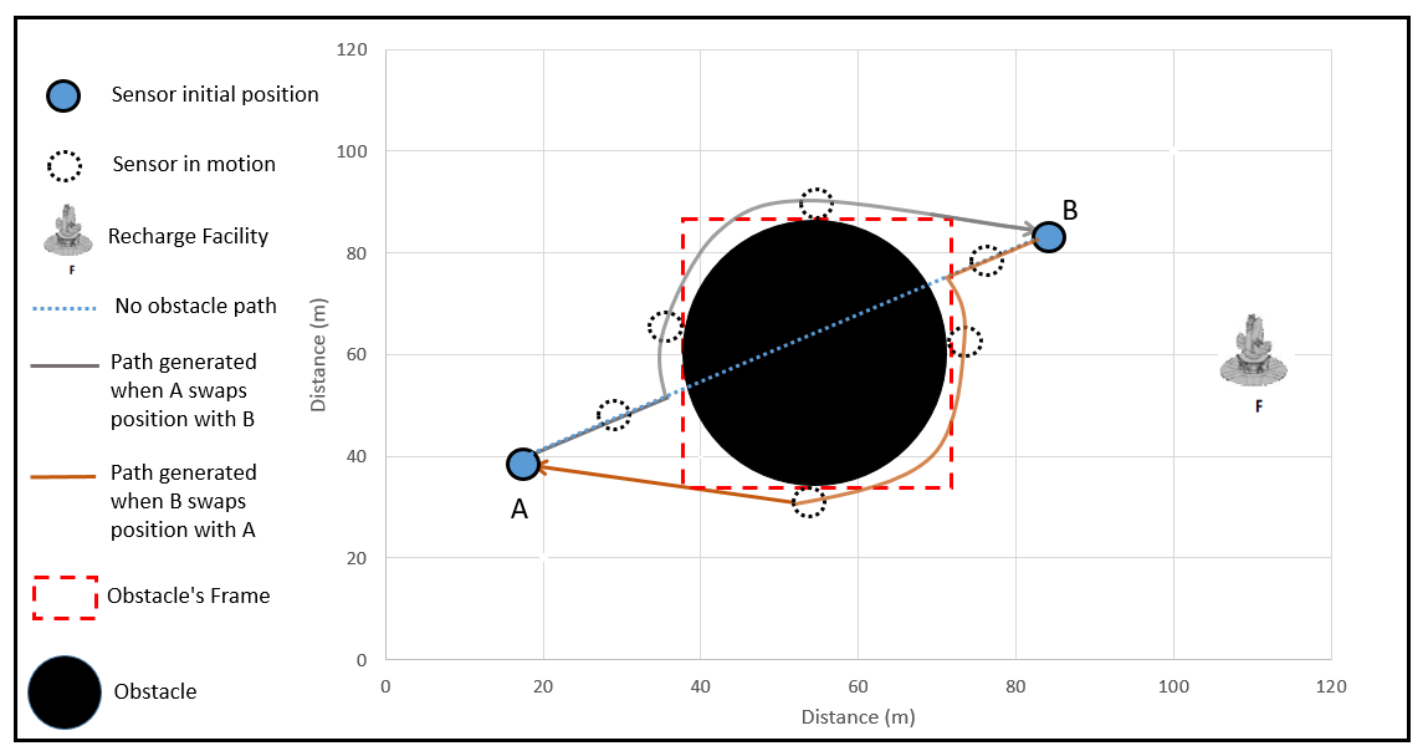

Figure 3.2: Discovering the Obstacle while Swapping Positions.

Figure 3.2 shows the swapping operation. In this case, when the sensors are swapping 
positions the obstacle is reached from both sides of the straight line that joins the two sensors. Since sensors move always in the same direction (i.e., clockwise) each sensor will avoid half of the obstacle. While a sensor moves around the obstacle it will update the four values that represent the obstacle. When each sensor arrives at the destination position, a SWAP_COMPLETE message will be sent along with the values

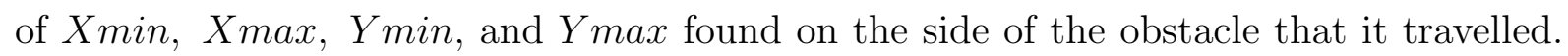
Once the sensor receives the SWAP_COMPLETE message it has all the necessary information to generate a rectangular frame that covers the known parts of the obstacle.

\subsection{Using the Obstacle's Frame to Generate a Proactive Path}

Sensors keep a small quantity of information about the obstacles. This information can be used to avoid unnecessary exploration and to try to generate a shorter path. If a sensor has information about an obstacle and the obstacle's frame has an intersection with the straight line that joins the sensor with its target, the sensor can use the information to avoid the obstacle. The sensor calculates which corner(s) of the rectangle are the closest to the sensor and its target without intersecting with the obstacle. Then the sensor will generate a path that contains its initial position, the closest corner(s) and the target position. In the worst case, the sensor will need to visit two corners before reaching the target.

The sensor can also share information about the obstacle frames with its neighbours. It uses the SWAP_COMPLETE, SWAP_REQUEST, SWAP_ACCEPT and the RECHARGE_DONE messages to share this information. This allows sensors to avoid unnecessary exploration, and generate safe paths (paths that are supposed to avoid obstacles) .

Figure 3.3 shows how sensor $A$ discovers the obstacle and generates the obstacle's frame. When sensor $A$ returns from the recharge station, it sends a RECHARGE_DONE message that contains the discovered information about the obstacle. This information 


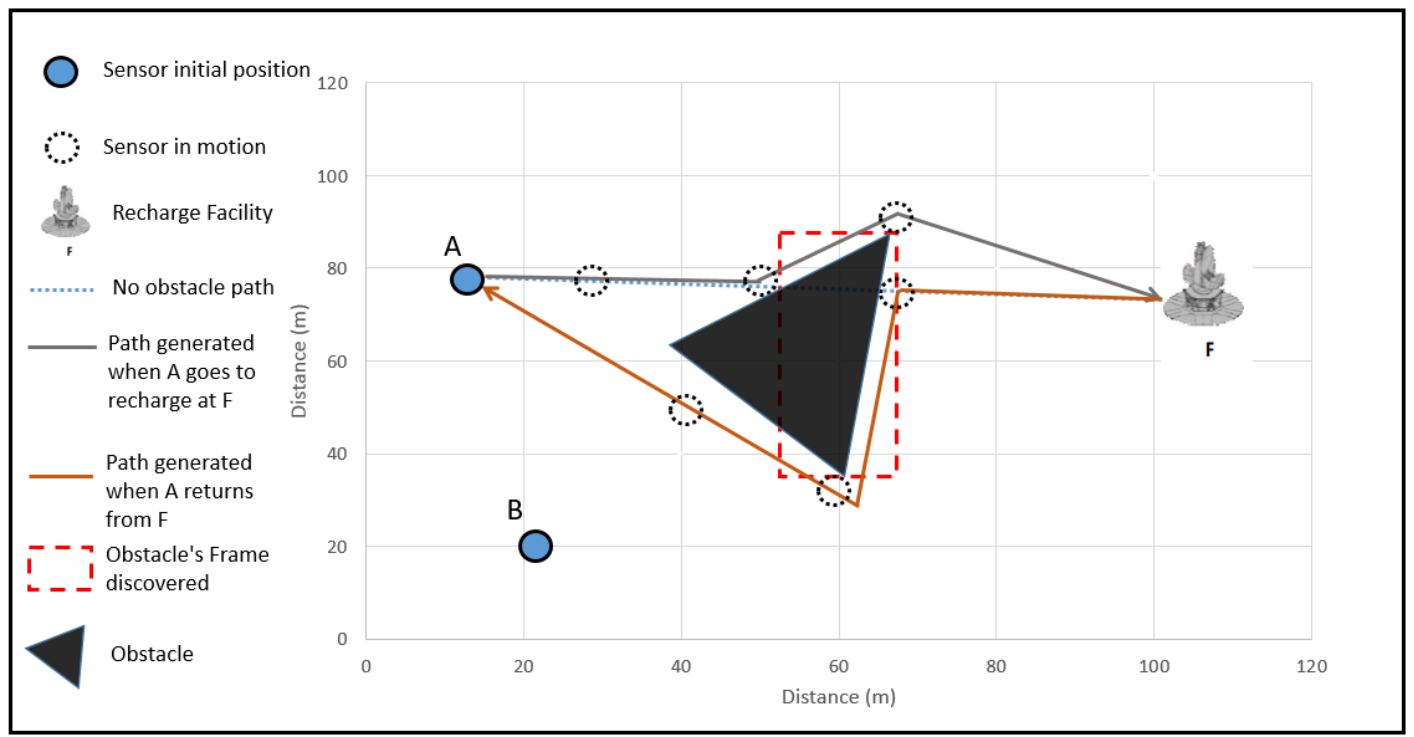

Figure 3.3: Discovering the Obstacle while Recharging.

is used by sensor $B$ in Figure 3.4 to generate a path to the recharge facility that includes the closest corner of the frame to $B$ and $F$ without intersecting with the obstacle. By using the frame, $B$ avoids unnecessary exploration and generates a safe, and short path.

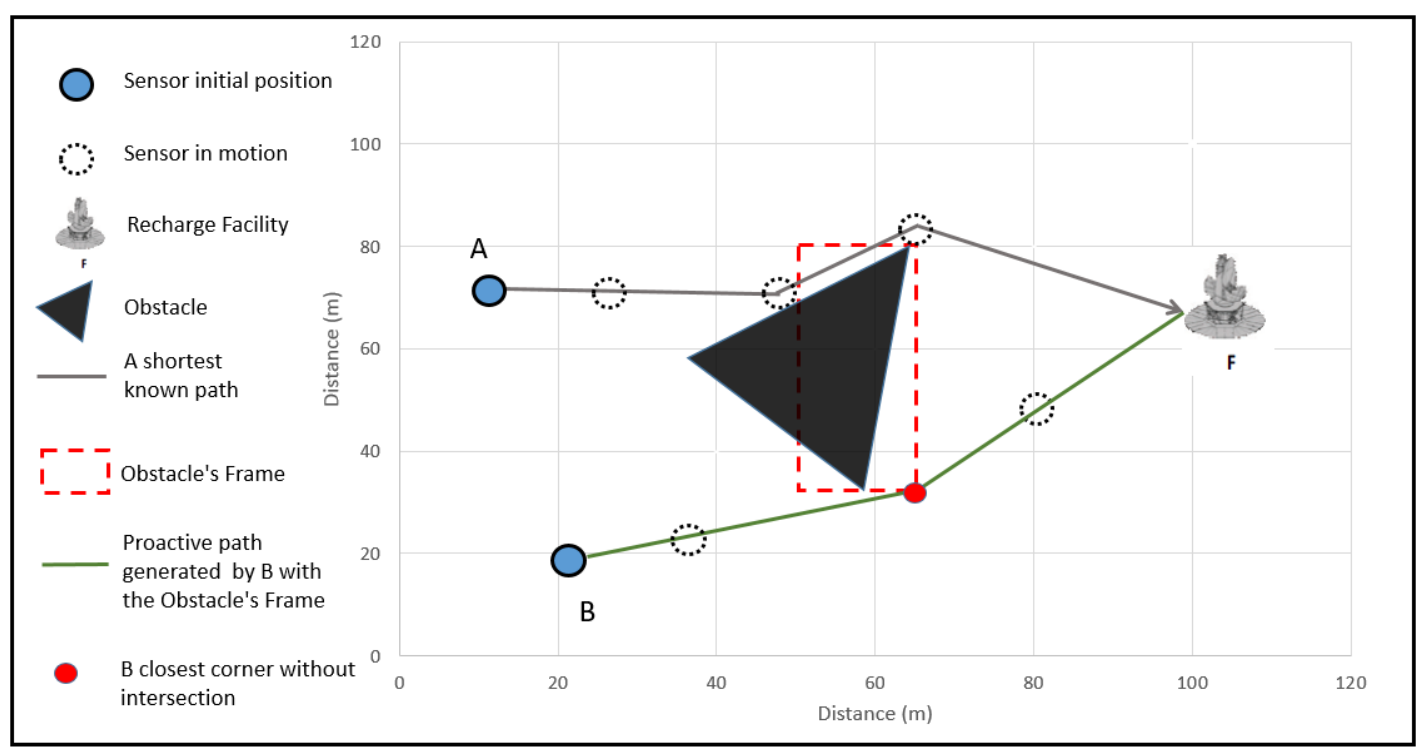

Figure 3.4: Using the Obstacle's Frame to Generate a Safe Path.

Every time a sensor discovers an obstacle or receives information about a new one, it will consider the paths to its neighbours, and to the recharge station, and see if this new information can improve known paths or generate shorter paths in unexplored regions. 


\subsection{Improving the Obstacle's Frame}

There are several cases where the obstacle frames generated by sensors do not contain the complete obstacles. Figure 3.3 shows an example of a generated frame that does not contain the entire area of the obstacle. This would not have an impact on the path generated using the frame information shown in Figure 3.4. But there are cases when the sensor is travelling to the closest corner and reaches a part of the obstacle that was not contained in the frame. When this happens, the sensors avoid this new found obstacle while trying to reach the closest known corner of the frame. Then it uses the new information to improve the known obstacle's frame. Figure 3.5 and Figure 3.6 show how the obstacle's frame is improved in cases when this occur.

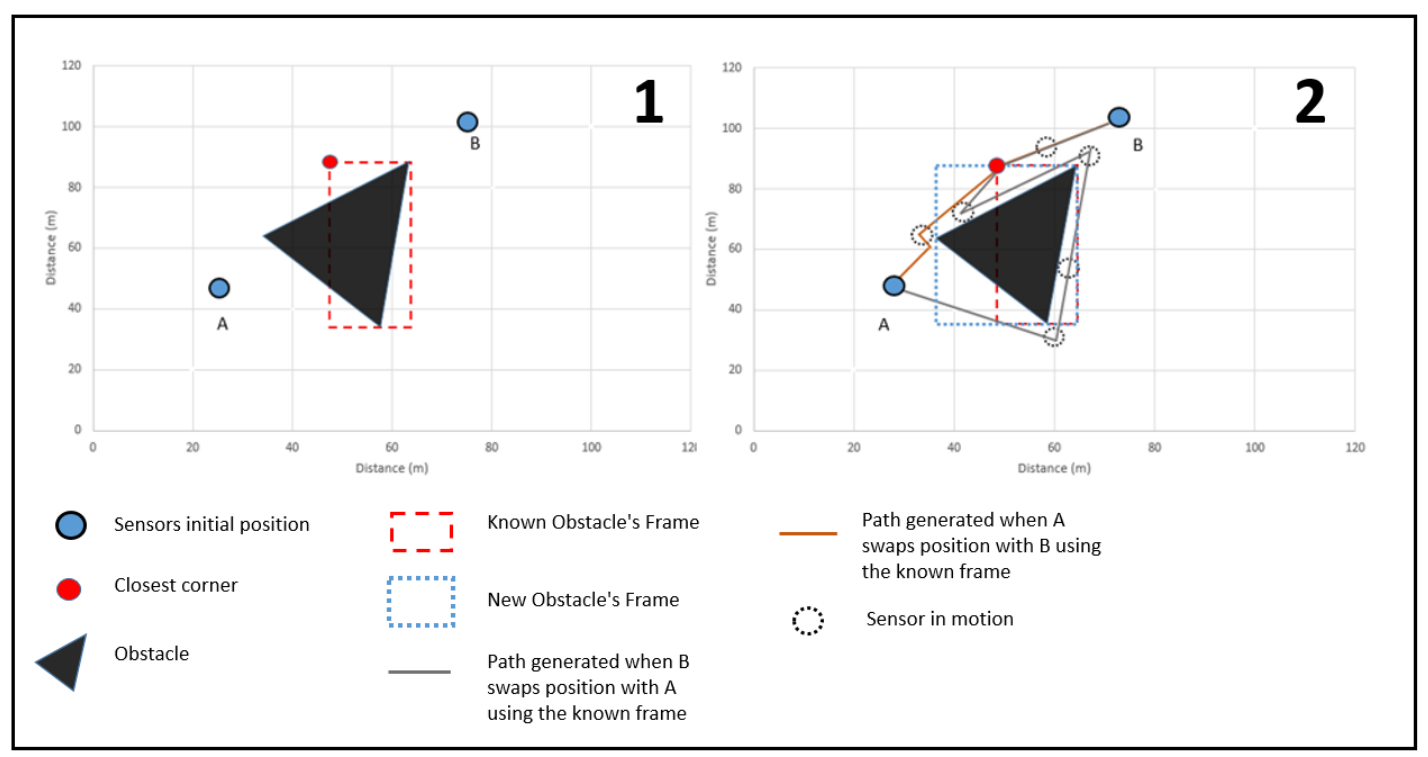

Figure 3.5: Improving the Obstacle's Frame Accuracy.

In Figure 3.5 (1), the two sensors know the obstacle's frame and they use this information to swap positions by calculating the closest corner that permits them to navigate around the frame without intersecting it. They create a path that contains the starting original position, the frame corner, and the target. When they travel along the created path, they reach an obstacle because of the frame that does not contain all of the obstacle. Figure 3.5 (2) shows this situation. Both sensors avoid the obstacle and generate a new obstacle frame that contains the previous frame. When sensors discover a new frame that has an intersection with, or contains earlier frames, they will always keep the most recent obstacle information, and delete the earlier information about the obstacles. Then 
in Figure 3.6 (3) when sensors $A, B$ want to swap positions again, they use the new obstacle frame to calculate a new path. If the cost of the new path is less than the cost of the stored path, they will travel the new path; otherwise, they will travel the shortest known path. By comparing any newly discovered path with the best known path, sensors improve their paths with new information about obstacles.

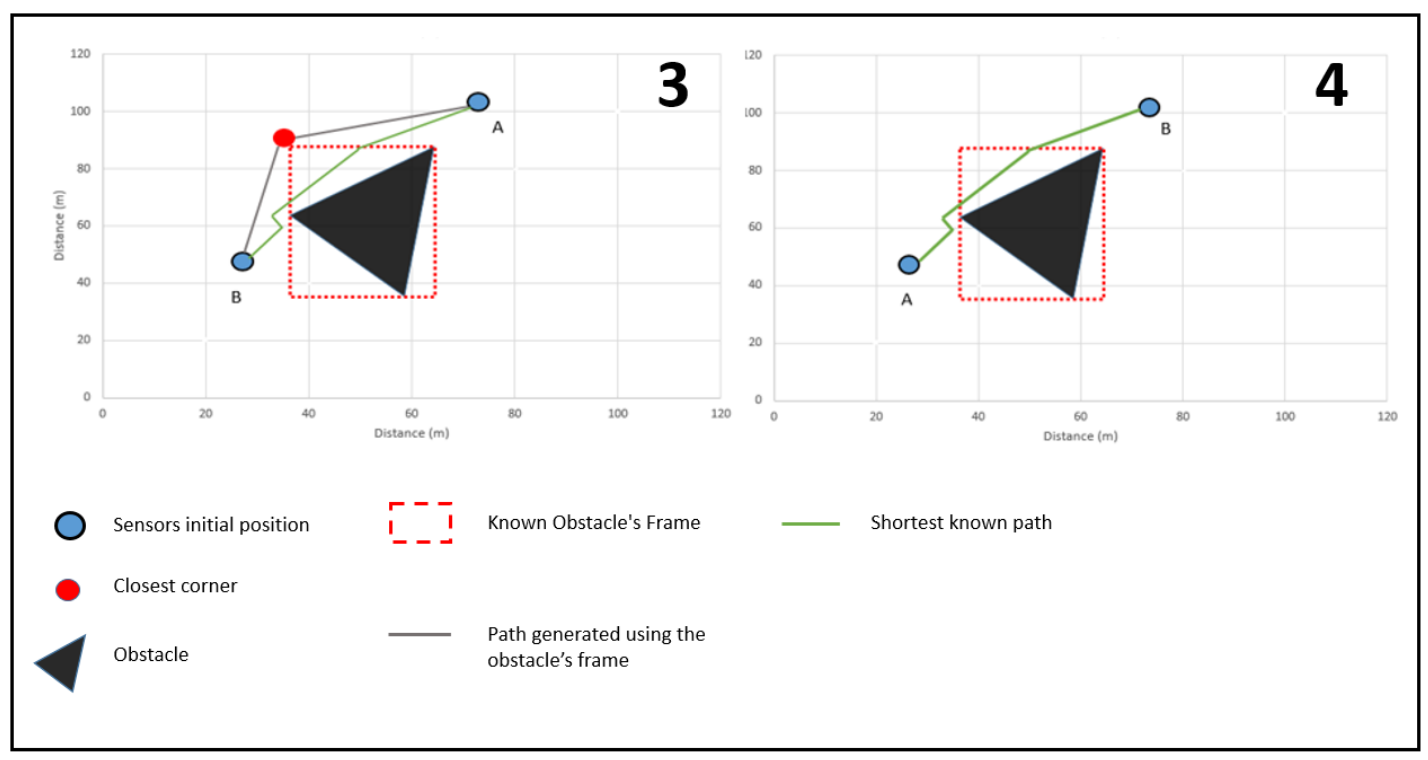

Figure 3.6: Using the New Obstacle's Frame Information.

Sometimes, paths contain more than one obstacles. In these cases, the sensors will see this obstacle's frame as a big obstacle. This representation can produce an unnecessary waste of energy when a sensor travels to the closest corner of the frame to avoid the obstacles. The way our proposal deals with this problem is presented in the next example.

Figure 3.7 shows the scenario where a sensor $A$ finds two obstacles while en route to the recharge its battery. The information gathered by the sensor $A$ produces an obstacle frame that contains both real obstacles. The new obstacle's frame information will be received by sensor $B$ and $C$. Sensor $C$ will use this information to calculate the closest corner and generate a safe path. In the case of sensor $C$, the path generated with the obstacle information is not the shortest path that $C$ can generate. But using the obstacle information $C$ avoids doing some unnecessary exploration, Figure 3.8.

In sensor $B$ 's case, when it receives the information about this obstacle frame, it detects 


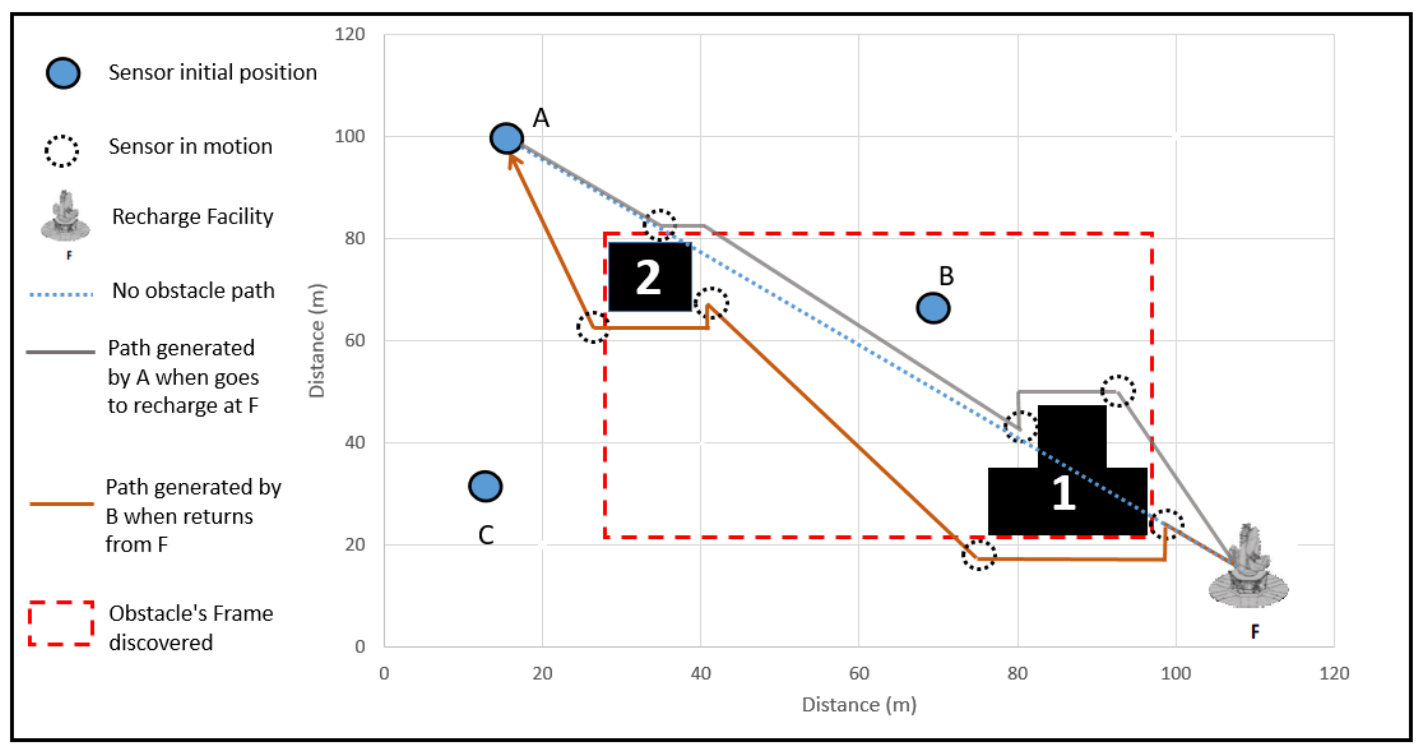

Figure 3.7: Discovering the Obstacles.

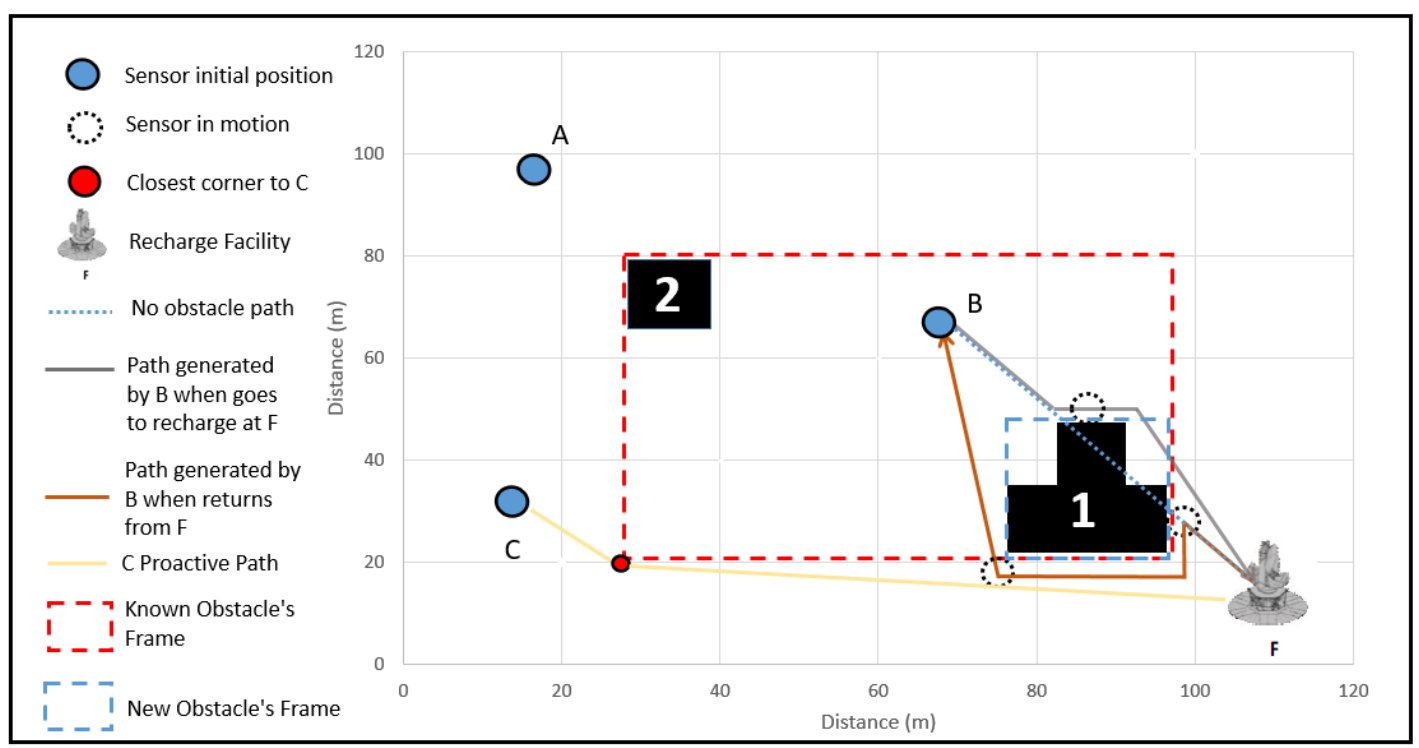

Figure 3.8: Improving the Known Obstacle's Frame.

that it is inside the frame. The sensor deletes this false information because the sensor cannot be deployed over an obstacle. Then when sensor $B$ recharges its battery at the recharge facility, it will discover one of the two obstacles. When it does this, it will share the new information with its neighbours. Figure 3.8, and Figure 3.9 show how sensors use the new information to improve the paths.

When sensor $A$ receives the new obstacle information, it detects that the new frame is contained by the one it already has, so it deletes the large frame it had previously stored. 


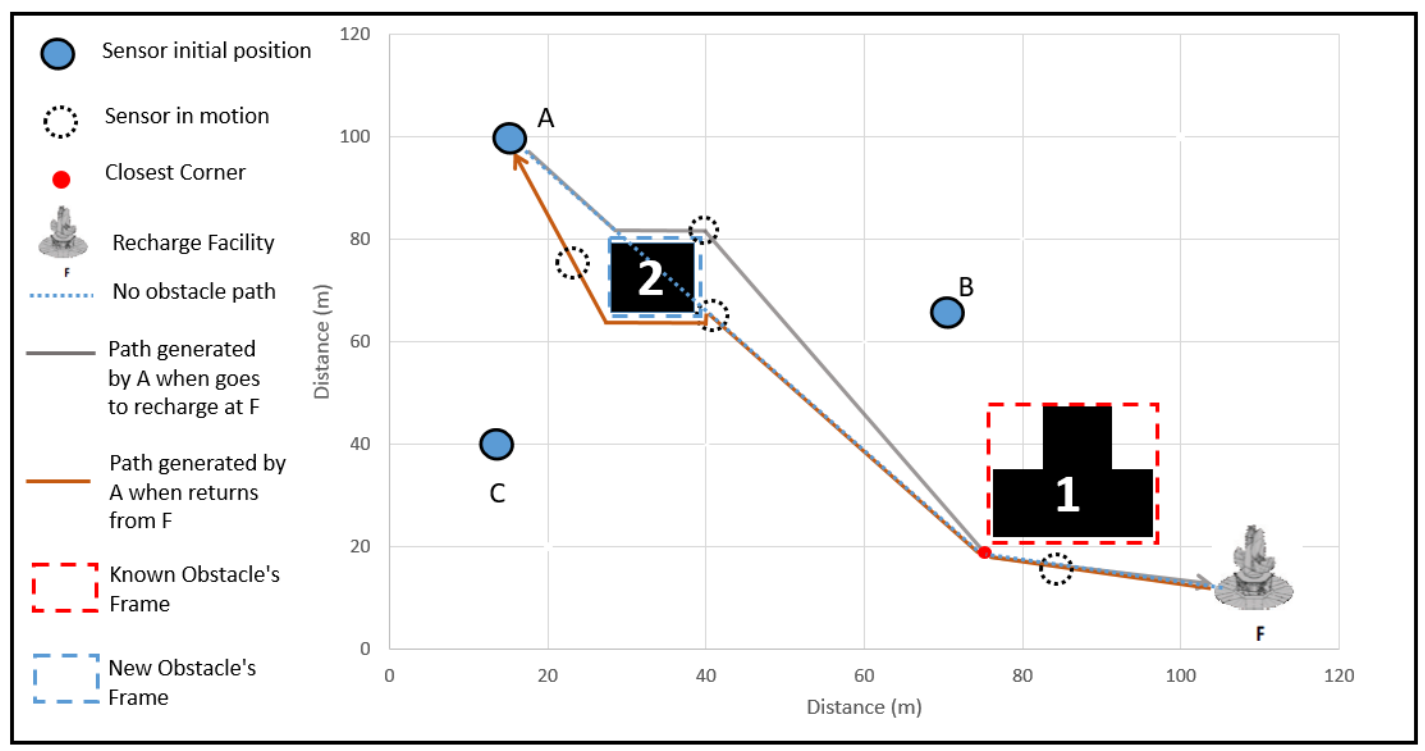

Figure 3.9: Using the Known Obstacle's Frame.

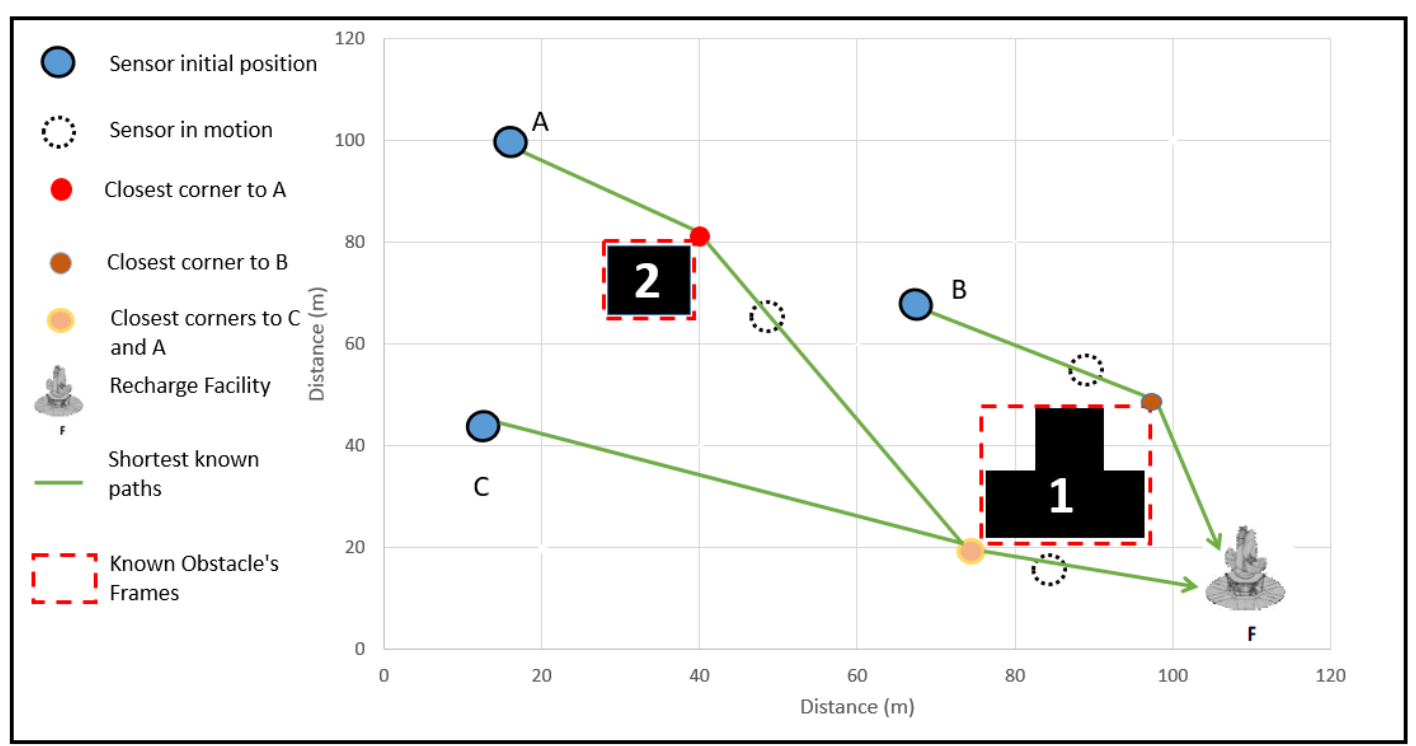

Figure 3.10: Generating Short and Safe Paths.

Later, when $A$ needs to recharge its battery, it will use the new information to calculate a safe path. Figure 3.9 shows the case when sensor $A$ travels to the recharge station using the closest corner of obstacle " 1 " and discovers obstacle " 2 " in the process. At the end of the trip, both obstacles are discovered and the obstacle frames are adjusted to the shape of the obstacles.

In this same example, when sensor $C$ receives the new information about the obstacles, it will delete the big obstacle frame. With the new frames, adjusted to the real obstacles, 
sensor $C$ generates a new path to the recharge station. It will use the closest corner of the frame for obstacle " 1 " to generate a safe path and it will keep the shortest path. Sensor $A$ will use both frames to generate the shortest path to the recharge station. Figure 3.10 shows how sensors $A, B, C$ use the frames to generate short, and safe paths to the recharge facility.

\subsection{Resolving the $\mathrm{U}$ and $\mathrm{H}$ Shape Problem}

In chapter 2 it was mentioned that the IBA algorithm fails when the obstacle has $\mathrm{U}$ or $\mathrm{H}$ shape. The algorithm fails due to its goal-oriented characteristic. Figure 3.11 shows a scenario where the IBA algorithm produces a loop and the sensor will never avoid the obstacle. In this case, when sensor $A$ arrives at the position $X_{i}, Y_{j}$ it will move freely in the direction to the recharge station. Moving this way, the sensor is taken to a position on the edge of the obstacle that was already visited. The sensor will follow the obstacle's edges and it will arrive again at the position $X_{i}, Y_{j}$, where the loop starts again.

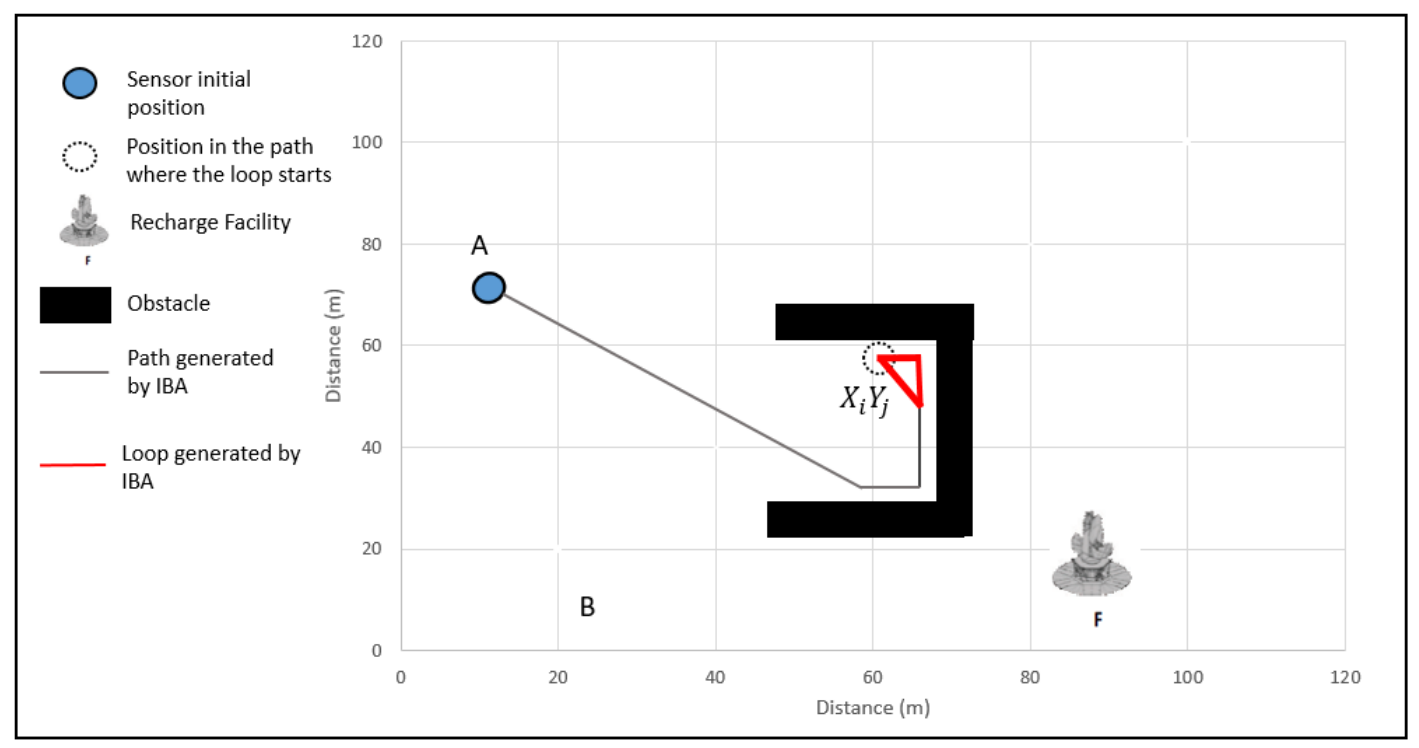

Figure 3.11: Dead Scenario with U-Shaped Obstacle.

With the proposed modification of keeping the four most extreme values of $X$ and $Y$ of the discovered obstacles, this loop can be avoided. Figure 3.12 illustrates this modification: it shows a sensor at the position $X_{i}, Y_{j}$ that wants to move freely in the direction of the 
recharge facility, but it detects that this movement will take place inside the obstacle's discovered frame. In this case, the sensor will keep following the obstacle's edges until:

i. The sensor is capable of free movement outside the frame and in the direction of the target (recharge station), that is positioned outside the frame.

ii. The sensor can move freely in direction of target position that is also inside the frame.

iii. The count of the turning angles (left and right) is equal to 0 .

The idea of counting the turning angles is used in a well-known algorithm to solve maze problems, called the Pledge Algorithm [1]. In the Pledge Algorithm, like in our strategy, the robots must always move in the same direction when an obstacle is reached. The correctness of this algorithm has been proved in several papers (e.g., [13]). In our case, the sensor will count the turns every time it reaches an obstacle. A sensor will only move freely inside the obstacle's frame if the count of the turning is 0 and the target position is outside the frame, or if the target position is inside the frame. It is assumed that turning right will increase the counter by 1 and turning left will decrease the counter by 1 . When free movement outside the frame of the obstacle is re-established, the count is reset to 0 .

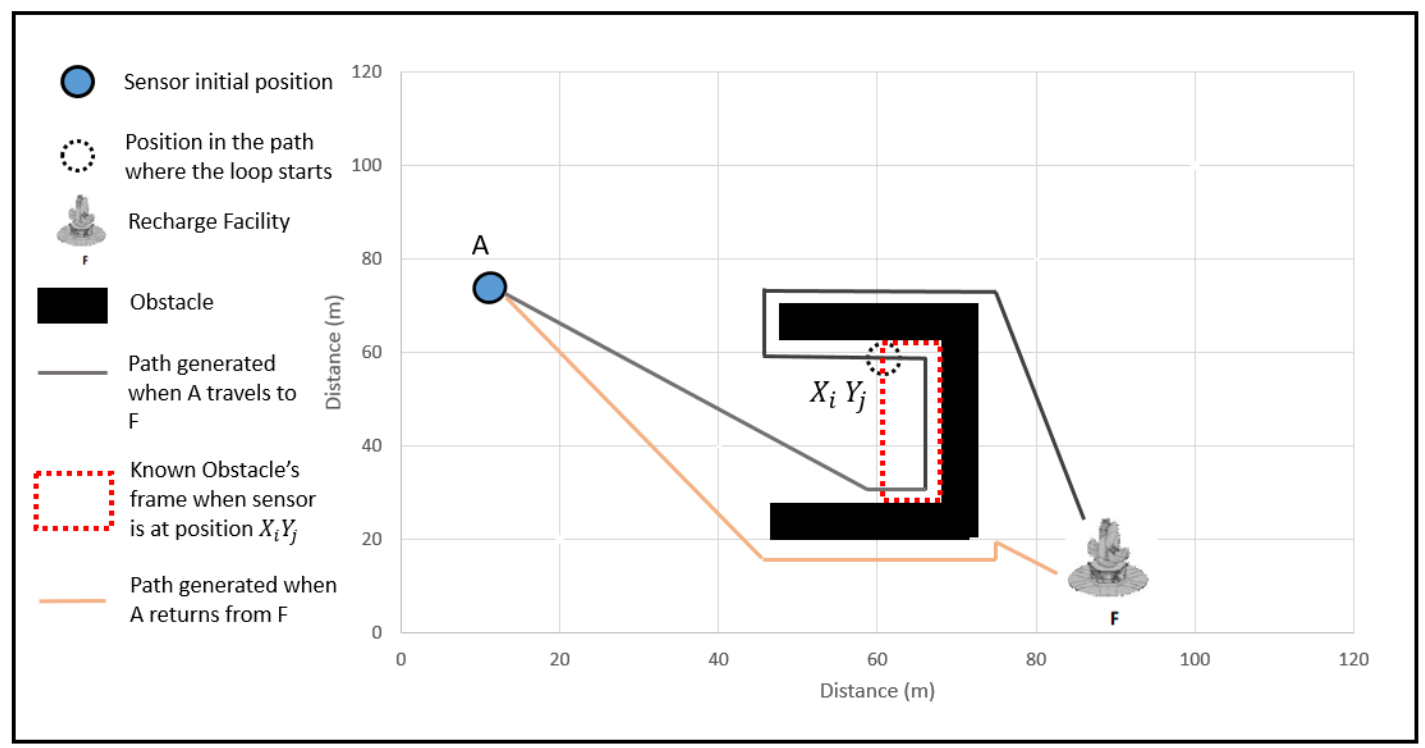

Figure 3.12: Avoiding the U-Shaped Obstacle.

Figure 3.13 shows how, by using the frame information, sensor A can generate a shorter path that avoids entering inside the U-shaped obstacle. The information about the 


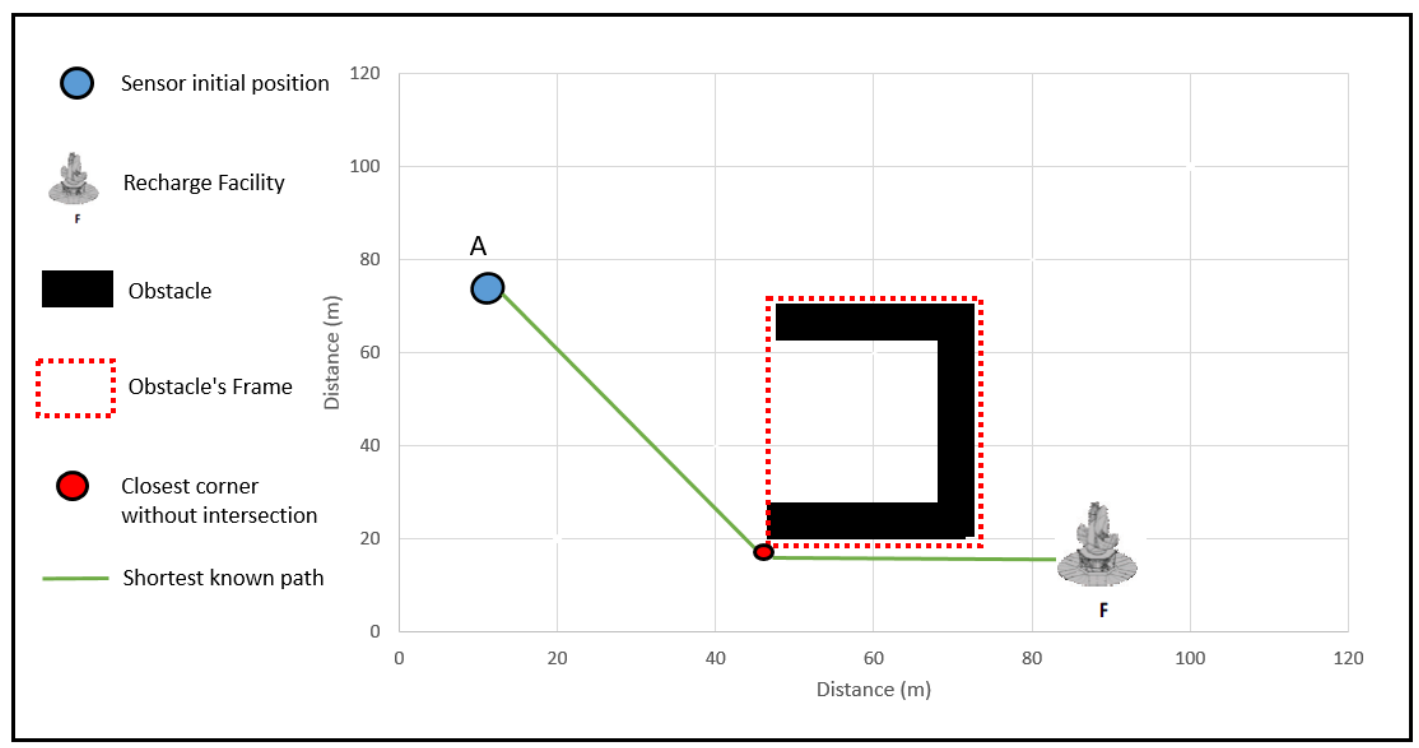

Figure 3.13: Using an Obstacle's Frame to Generate a Short Path.

obstacle's frame can be used by the sensor to improve its path and can be shared with other sensors, allowing other neighbours to avoid the U- and H- shaped obstacle traps.

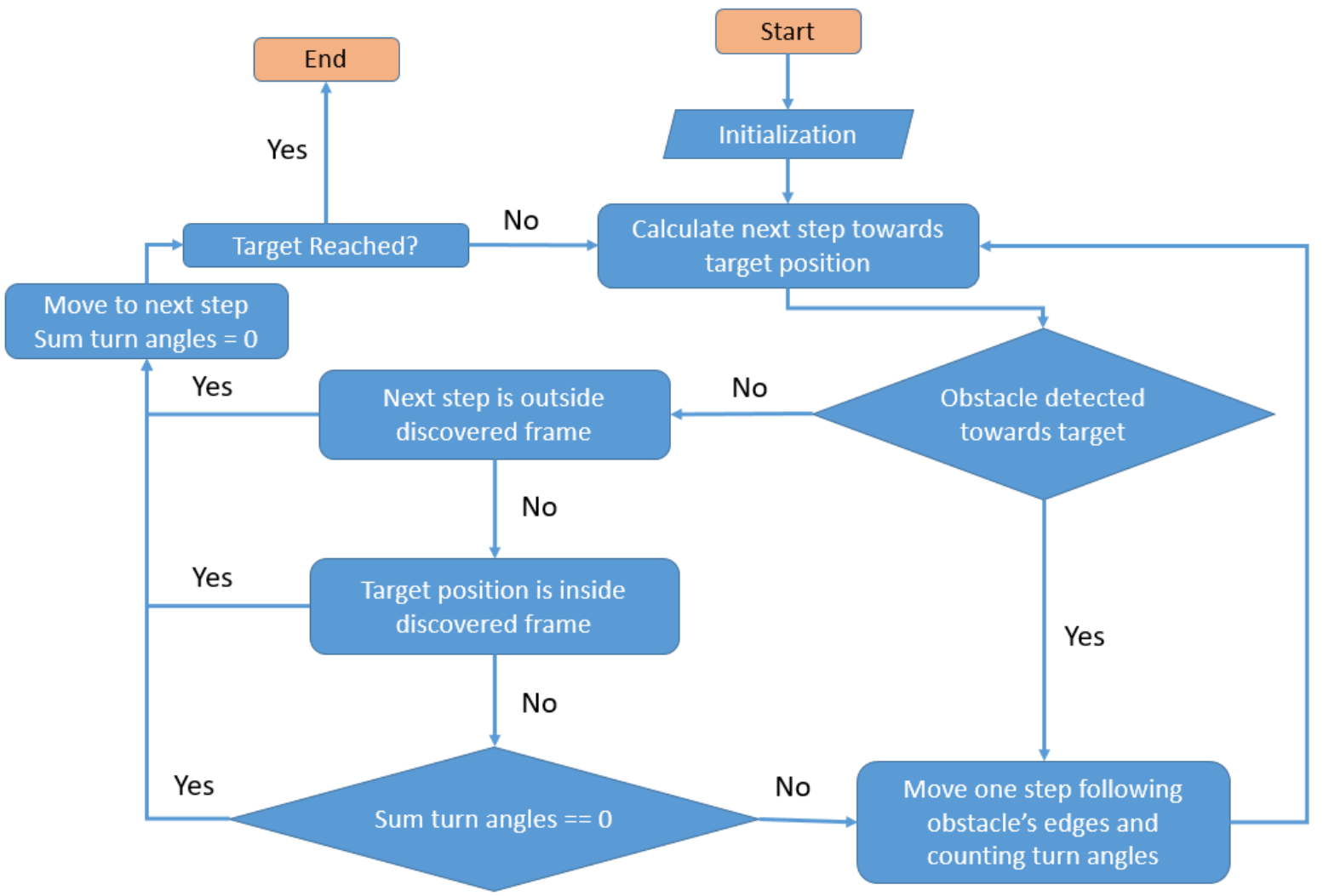

Figure 3.14: Flowchart of the IBA Modified Algorithm. 
Finally, Figure 3.14 shows the flowchart of the IBA modified algorithm that uses the discovered frame, and the total count of turning angles.

\subsection{Polygonal Mazes}

By introducing the idea of counting the number of turning angles, the new modified IBA algorithm is not only able to avoid but also to lead a sensor out of obstacles in the shape of a polygonal maze. This improvement allows our application environment to be enriched with obstacles in the form of polygonal mazes. It is important to remark here that in scenarios where the target position is inside the maze, the Pledge Algorithm fails. In these cases, it does not guarantee the sensor will reach the desired position. In our model, when a sensor detects that the target position is inside an obstacle's frame it will try to reach the position by moving freely even if the count of the turning angles is not equal to 0 . In some simple scenarios with $\mathrm{U}$-, or H-shaped obstacles, the sensor will reach the target position even when the target is inside the frame. However, in more complex obstacles like polygonal mazes, the sensor could die while trying to reach the target position inside the maze. In our approach when this problem occurs the sensor will send a SENSOR_OUT message containing the maze's known frame before dying. With this information, other sensors can avoid entering the maze, and wasting time and energy trying to solve it.

Figure 3.15 shows when a sensor that is deployed inside a maze needs to recharge its battery, it can reach the recharge station using the count of the turning angle and the discovered frame to solve the maze. However, when it tries to return, the sensor can not reach the initial position, and eventually dies. After the sensor has died, all its neighbour sensors in range of communication will know the frame that contains the maze, therefore they will able to avoid it.

An special case is when the recharge station is deployed inside a maze, in this case all sensors could die trying to reach it. That is why in our model the recharge station has to be deployed in a position where it can be reached in straight line from at least one point of the frame that contains all the obstacles. Otherwise, it can not be guaranteed that sensors will survive. 


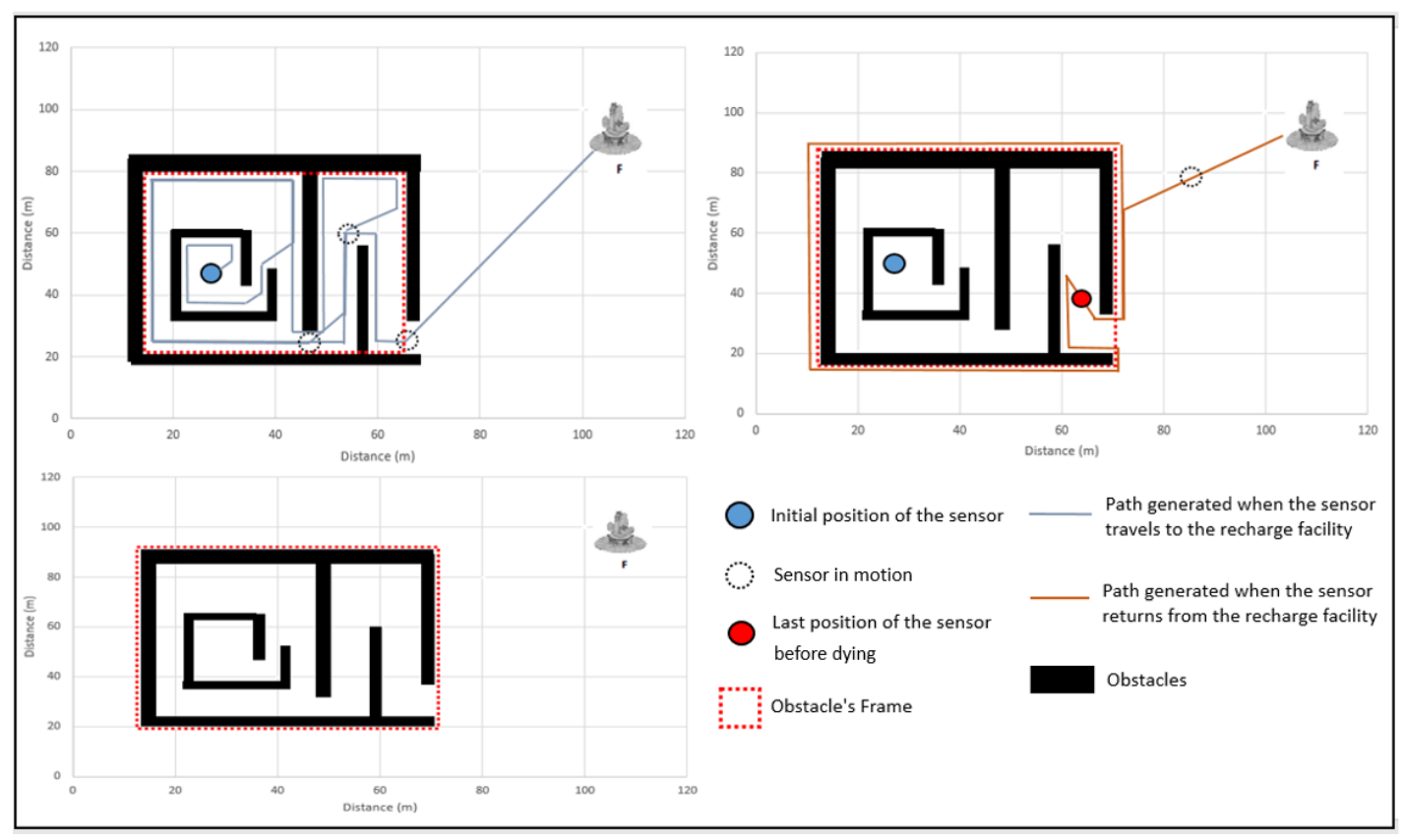

Figure 3.15: Worst-Case Scenario for a Sensor Inside a Polygonal Maze.

\subsection{Summary}

With the proposal of storing the values of Xmin, Xmax, Ymin, and Ymax while avoiding obstacles using the IBA algorithm, sensors can improve the paths between neighbours and the paths to the recharge station. With this information, sensors can pro-actively avoid obstacles and avoid unnecessary exploration. By using frame information and counting the turning angles, sensors can avoid obstacles that have U and $\mathrm{H}$ shapes. In addition, sensors are able to avoid and solve polygonal mazes when the target position is not inside the maze. Figure 3.16 shows how using the obstacle frame information, and all the modifications proposed sensors are able to avoid complex obstacles and generate short, and safe paths.

Sensors will have routing tables with the paths to their neighbours and children, and they will now have a list of known obstacle frames. It is important to remark that when two sensors swap positions they exchange the information in their routing tables but the list of known frames and sensors IDs do not change. Sensors share obstacles information by including the values of $X \min , X \max , Y \min$, and $Y \max$ of the known frames in 


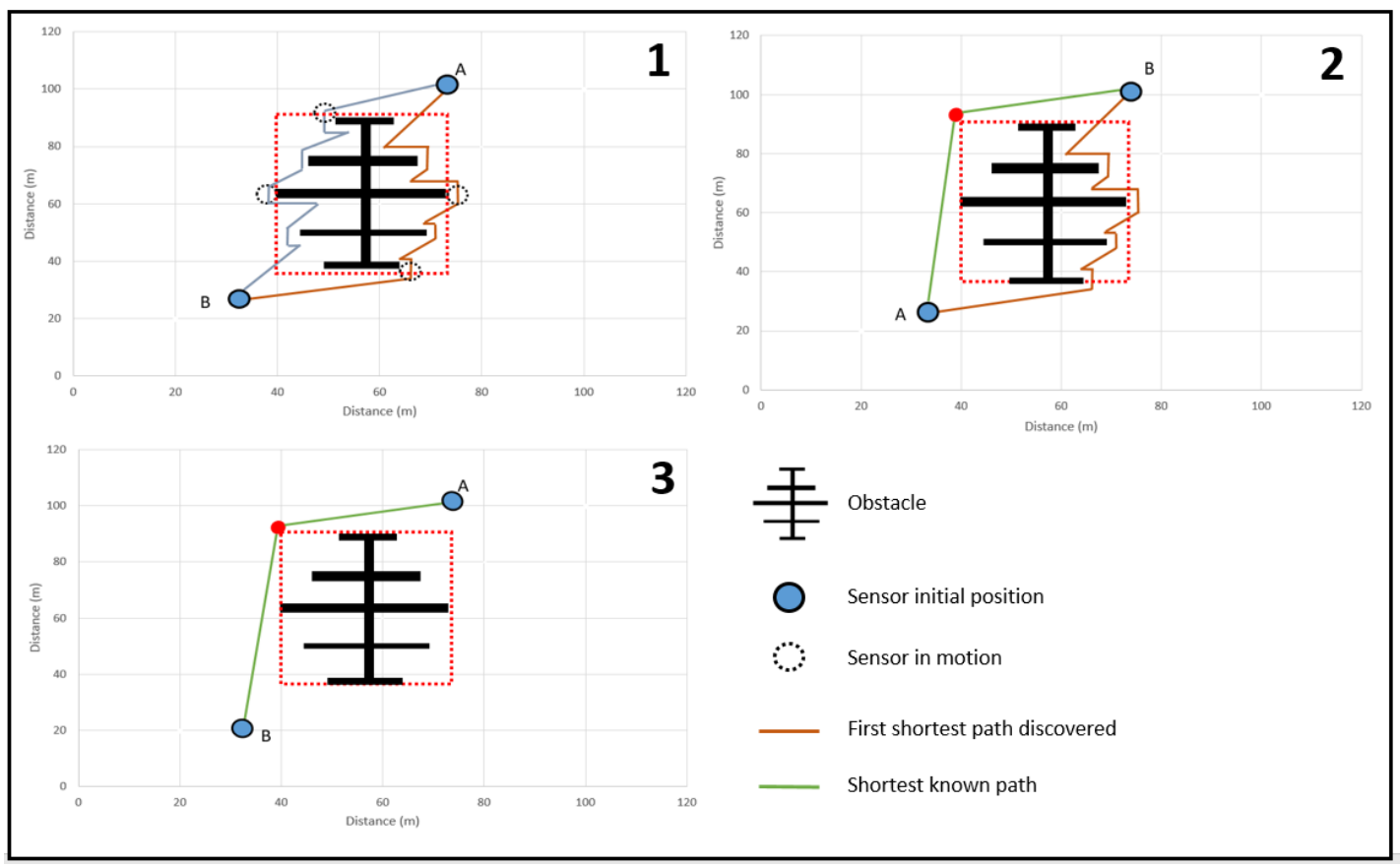

Figure 3.16: Improving the Path Between Sensors.

the following messages sent to their neighbours ( SWAP_REQUEST, SWAP_ACCEPT, SWAP_COMPLETE, RECHARGE_DONE). When sensor A receives new information about the obstacle's frame in any of these messages, the following different situations can occur:

i. If the new frame intersects with a frame known by $A$, then sensor $A$ will not add the new frame to the list of known frames.

ii. If the new obstacle frame contains a frame know by $A$, then sensor $A$ will not add the new frame to the list of known frames.

iii. If the new obstacle frame is contained in a frame known by $A$, then sensor $A$ will delete the known obstacle frame and will add the new one to the list.

iv. Otherwise, the new frame information will be added to the list of known frames.

The information about the obstacles (frames) are transmitted in the same messages used by the energy restoration strategy to maintain the CDG, and coordinate the operations. Therefore, there is no need of using extra energy to keep the sensors information update. 


\section{Chapter 4}

\section{Experimental Results}

In this chapter, some experimental simulations to validate the impact of the modifications proposed in chapter 3 are examined. In addition, the passive and proactive strategies to restore the energy in sensor networks described in section 2.1 with the new modifications proposed in chapter 3 are evaluated in the presence of physical obstacles.

\subsection{Environment and Considerations}

The different experiments were implemented in the simulator Omnet++ [33], using the INET framework extension [34]. The recharge station, the obstacles, and the sensors were deployed over an area of $1000000 \mathrm{~m}^{2}$. For all the simulations, the obstacles were placed in fixed positions and the sensors and the recharge station were randomly deployed over the remaining empty spaces. In each simulation, 100 sensors and 1 recharge station were deployed. The recharge station was deployed guaranteeing that it was not inside a maze.

The sensor transmission range was fixed at $200 \mathrm{~m}$ [36]. The cost of basic operations like sending and receiving a message, moving, or being idle are the same values selected in [35]. The energy necessary to send a message over 1 unit of distance will be a constant $E$, and the energy necessary for receiving a message over the same distance will be $50 \%$ of $E(E / 2)$. These values are based on diferent results achieved in [36], where the cost of $E$ was set as $10 \mathrm{~mA}$. The locomotion cost is based on the length of the path (in meters) with a weighted factor of Cost of Moving (CM) for each unit of distance. The value of CM used is the same used in [35]. This value was calculated under the assumption that 
the amount of energy necessary for moving one unit of distance was 54X greater than the amount needed to send a message over the same distance.

The sensors will check their batteries at periodic intervals and after any operations. It is assumed that all operations consume energy. Each simulation was run for a $1 . E+05$ simulation seconds. In real time, each simulation took at least 1800 seconds depending on the number of sensors that survived and the strategy that was simulated.

The neighbours selection criterion used to construct the CDG was based on Gabriel Neighbours. The simulation results presented in [35] showed that an underlying topology based on Gabriel Neighbours achieved the best results.

For ranking the neighbours in order to know wich sensor should be asked first, different ranking method have been proposed. The ranking can be done based on the distance between the sensor and the neighbor, the neighbor' battery, or a combination of both. Due to the competitive results achieved in [35], it was also considered ranking the neighbours to swap position based on neighbour's distance (i.e., Closest First). The closest neighbour to the sensor will be the first to being asked to swap positions, then the second closer neighbour and so on.

\subsubsection{Performance Criteria}

The two main criteria used to evaluate the algorithms performance were whether or not the "state of equilibrium" is achieved, and the number of sensor losses suffered before the equilibrium is reached. As an additional consideration, the resources needed to achieve the ideal energy restoration were also analyzed. The analysis was focused on three key aspects: (i) the number of sockets of the recharge station, (ii) the sensor's speed, (iii) and the value of threshold that defined when a battery is in a critical state. The comparison between the total number of panic runs, and the total number of one-hop runs, was also used as a measure of the quality of the solutions. 


\subsubsection{Considerations About the Obstacles}

The obstacles used in the experiments have different shapes. The shapes of the obstacles were polygons, circles, and more complex obstacles (including $\mathrm{U}$ and $\mathrm{H}$ shape obstacles). Figure 4.1 shows the different obstacle shapes used in the simulation experiments.

The size of these obstacles could change, and with the purpose of being able to truly analyze the impact of adding obstacles in the field, it was decided to have two types of obstacles: obstacles having long perimeters, which require more energy to be avoided (large obstacles), and obstacles having short perimeters (small obstacles).

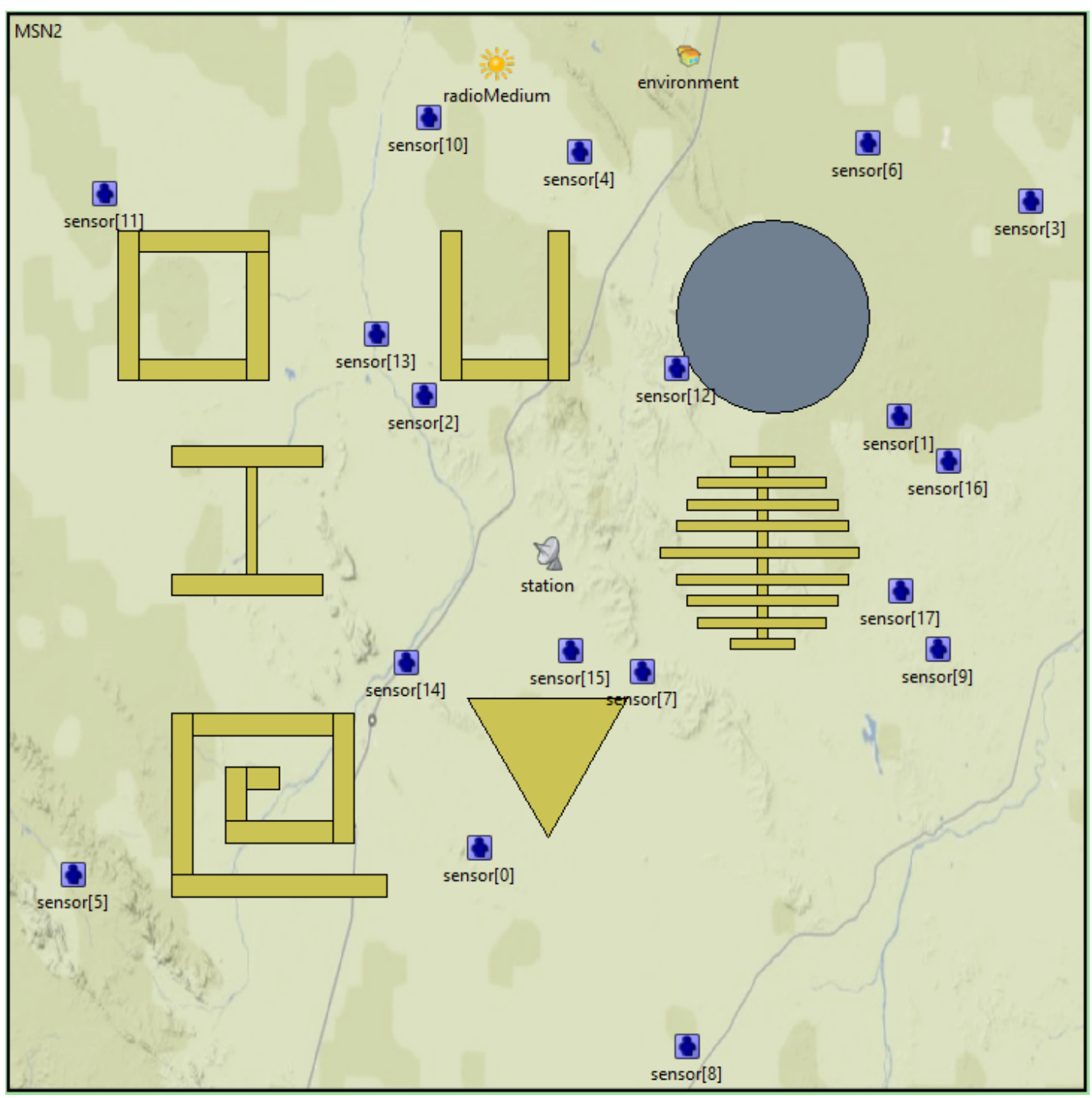

Figure 4.1: Different Shapes of Obstacles Used in the Simulations.

An obstacle is considered large if the energy necessary for a sensor to travel all the 
perimeter of the obstacle is greater than or equal to $50 \%$ of the total energy of the sensor in the BATTERY_CRITICAL state. Expression 4.1 shows when an obstacle is considered large in term of the size of the perimeter.

$$
P \geq 0.5(\alpha * \text { Total Battery }) / C M
$$

Here $\alpha$ is the threshold that defines when a battery is in critical state, and CM is the cost of moving one unit of distance. On the other hand, if the perimeter is smaller they are considered as small obstacles. Large obstacles have a big impact on the energy wasted by the sensor to avoid them, while small obstacles do not.

\subsection{Passive vs Proactive Obstacle Avoidance}

The first set of experiments attempts to find if both strategies reach the state of equilibrium in the presence of obstacles on the field. They also test which strategy achieves equilibrium with the fewest sensor losses. Another goal of this experiment is to test whether the findings of [35] are still true in the presence of obstacles.

In this experiment, 20 different deployments of sensors were tested all over the same scenario. The scenario contains 6 obstacles, 4 of them considered as large obstacles and 2 as small obstacles. The shapes used include $\mathrm{U}$ shapes, $\mathrm{H}$ shapes, circles and polygonal shapes. In this scenario, sensors will only use the obstacle's frame information to avoid the obstacles in $\mathrm{U}$ and $\mathrm{H}$ shape. They will not share the information and will not use the information to improve their paths.

Figure 4.2 shows that even in the presence of obstacles, both the passive and the proactive strategies to restore the energy in mobile network sensors achieve the state of equilibrium. The results show that the proactive strategy achieves equilibrium with fewer sensor losses than the passive. In the proactive approach on average $73 \%$ of the sensors survive, while in the passive less than $42 \%$. The graph also shows that the passive strategy reaches the 
state of equilibrium faster than the proactive. The behavior of the passive and proactive strategies in the presence of physical obstacles is similar to the behavior shown in [35].

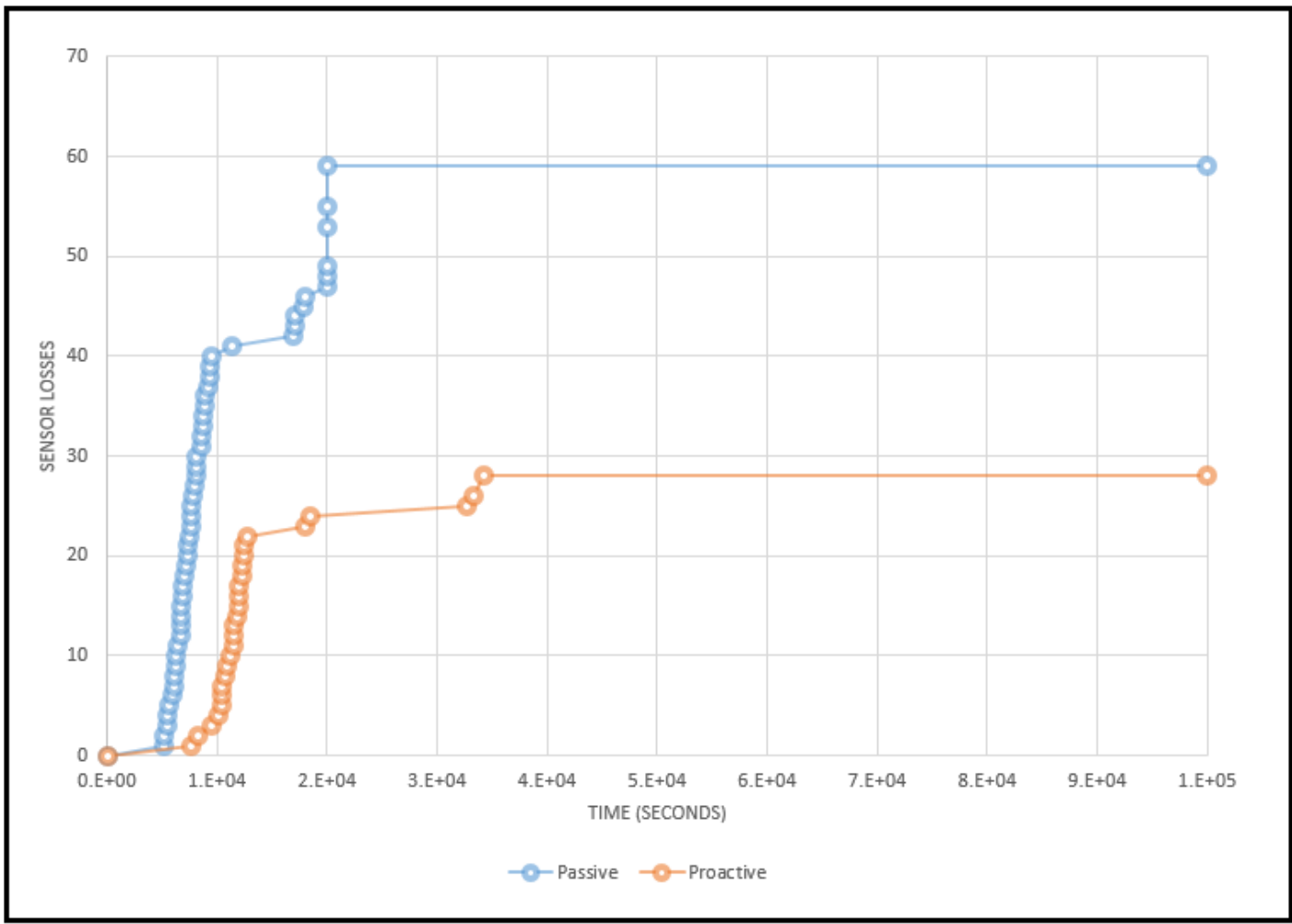

Figure 4.2: Passive vs Proactive Solutions in the Presence of Obstacles.

\subsection{Adding Obstacles}

The second set of experiments was done with the intention of discovering how the number of physical obstacles in the scenario affects the number of sensor losses. The obstacles used in this experiment were large obstacles. It is important to remark that when using the modified IBA algorithm to avoid obstacles, it was found in our simulations that sensors will travel around an obstacle's edges for less than half of the perimeter on average.

Figure 4.3 shows that the number of sensor losses increases with the number of obstacles on the field. Without obstacles on average $18 \%$ of sensors die in the proactive approach and $46 \%$ in the reactive approach. But, in the specific case of having 6 obstacles on the simulation scenarios, on average, $27 \%$ of sensors die in the proactive solution and $65 \%$ in the reactive approach. This increase depends on the type of obstacles and their positions. The biggest increase in the number of sensor losses happened when the newly 


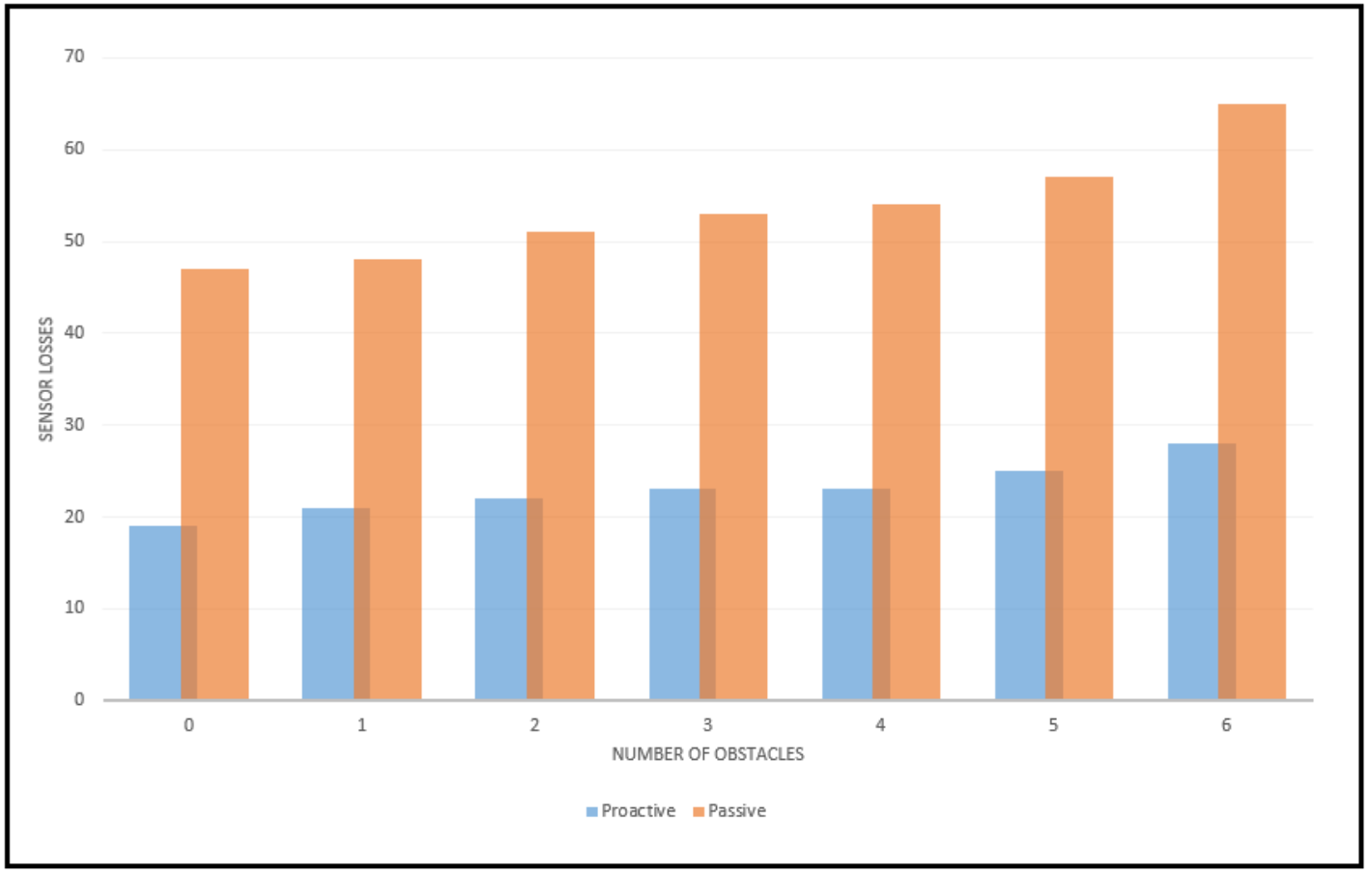

Figure 4.3: Adding Obstacles.

added obstacles created a scenario where the sensors needed to avoid more than one obstacle to swap positions or to travel to the recharge facility. These cases created the greatest number of sensor losses. In contrast, if the added obstacles were positioned in places where they did not interfere with the previous sensor paths, and avoid them did not consume an important amount of energy, there is a high probability the number of sensor losses will remain the same.

\subsection{Sharing vs No Sharing the Obstacle's Frame}

The third set of tests was performed to see whether sharing information improved the performance of the energy restoration strategies. Four tests were performed: passive and sharing information, passive and not sharing information, proactive and sharing information, and proactive and not sharing information. In the cases where the sensors shared information, they used this information to improve the known path and to avoid unnecessary exploration.

The first conclusion that can be drawn from the experimental results, showed in the 
Figure 4.4, is that using and sharing the information about the frames of the obstacles provide the best performance in the proactive strategy. Using the information, in the proactive strategy sensors reach the state of equilibrium with fewer sensor losses. This improvement is due to the number of swapping operations in the proactive solution being considerably higher than the number of recharge requests. It was observed that on average a sensor will swap positions 18 times before needing a recharge. In the first moments of the simulations, the high number of swapping operations permits the sensors to improve the frames of the obstacles and the paths between them. Then, when a sensor needs to recharge its battery, it should know the frames that best encapsulate the obstacles. With this information, sensors can produce a short and safe path to the station. The swapping operations also allow the sensors to improve the paths between them and to reach the optimal known paths in the first stages of the simulation. Then, when a sensor knows the optimal paths to its neighbours and to the recharge station, it can save energy while moving and the probability of survival increases. On average, sharing the obstacles' frame in the proactive solution decrease the number of sensor losses in $18 \%$.

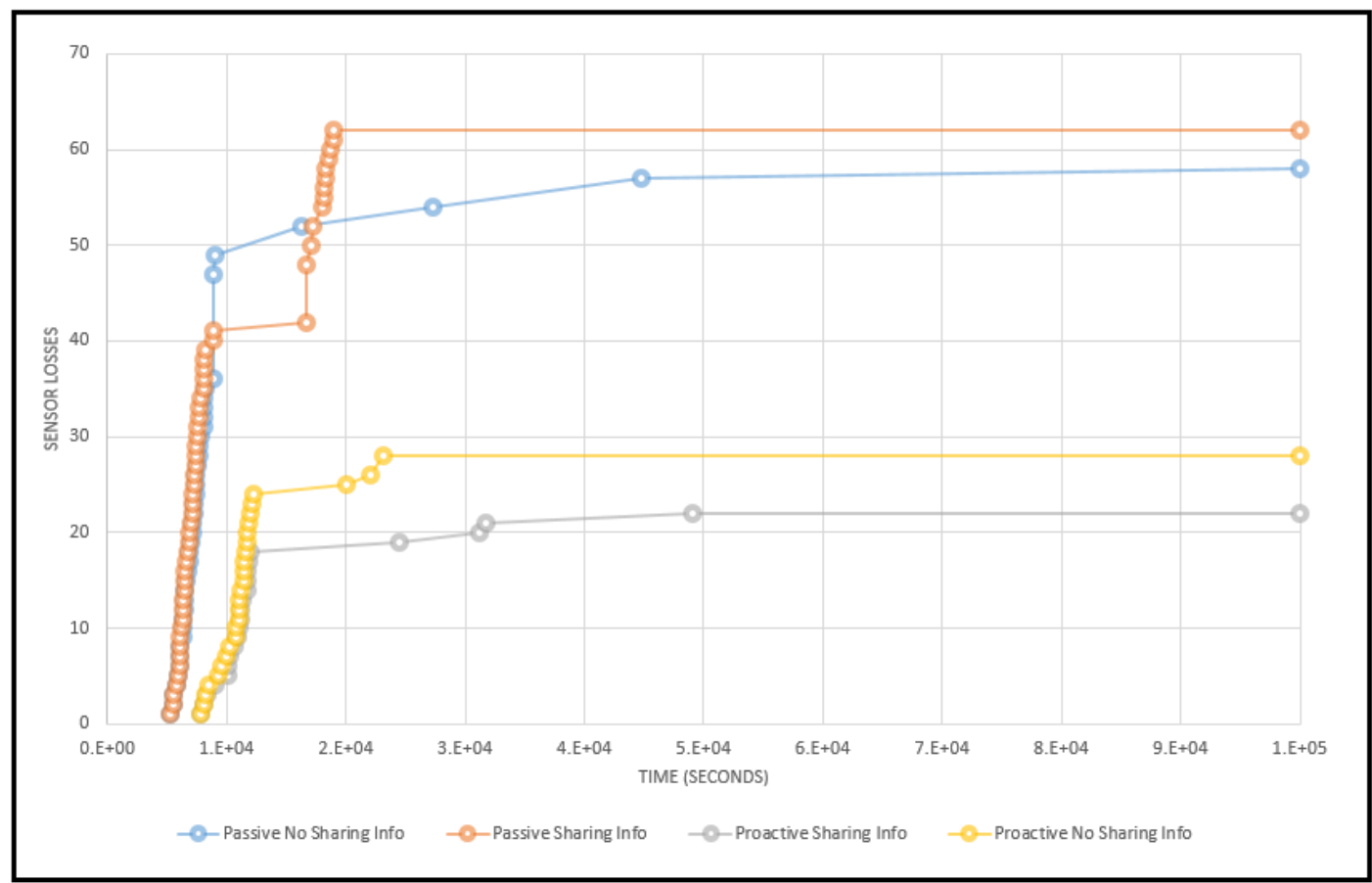

Figure 4.4: Sharing vs No Sharing Obstacles Information.

In contrast with the proactive strategy, sharing and using the obstacles' frame 
information in the passive solution led to worse results than when the sensor did not use the information. The reason for this behaviour is that in the passive strategy the process of improving the obstacle information only takes place when a sensor returns from the recharge station and shares the information. In a scenario that has several obstacles, a sensor that travels to the recharge facility and reaches more than one obstacle creates a big frame that contains both obstacles. This big frame is used by its neighbours to avoid the obstacles while travelling to the station. The only way to improve this information in the passive strategy is for a sensor deployed inside the frame to recharge its battery at the station and discover one of the obstacles inside the frame. When this happens, only the neighbours inside the sensor's transmission range will improve the obstacle's frame information. Sensors outside the transmission range will need a common neighbour that received the new frame to get recharged before them and to share the improved information. Alternatively, they need to survive more than one recharge time in order to allow the information to arrive to them.

The problem is that, in the passive strategy, more than $95 \%$ of the sensor losses occur before sensors receive their first recharge. This means that they never reach the recharge facility. The process of improving the information, therefore, takes too long and some sensors die because the frame that they use to avoid the obstacles makes them travel a long and expensive path.

In the passive strategy improving the paths does not provide a significant advantage because if there is a path to the station it can be assured with very high probability that the sensor will survive. In the passive strategy more than the $95 \%$ of the sensors that are able to recharge one time survive. The only benefit of improving the path is that after the second recharge of a sensor, the station's socket will be free earlier. But, since almost all sensor losses happen before sensors get their first recharge, no advantage can be gained from this improvement.

In both passive and proactive strategies, the information about the obstacle is transmitted and updated using the same messages that the energy restoration strategy needs to work. This means that the sensors do not need to use any additional energy to transmit messages with the obstacles information. 


\subsection{Travelling vs No Travelling the Graph}

This experiment tests whether it is better for a sensor in panic run mode to travel the graph (CDG) created by the energy restoration strategy to the recharge station, or to take a direct but unknown path. In such situation, the unknown path might be shorter, but if there are multiple obstacles or an obstacle with long perimeter, the sensor would have a better chance of survival by travelling the graph.

Figure 4.5 shows the simulation results of this experiment in environments where the number of obstacles was 6 , and 4 of them had long perimeters. The results show that in environments where there are several obstacles, it is better to travel the graph than to move directly to the recharge station. Sensors travelling the graph use the known path between them to reach the recharge station safely instead of trying to discover the direct path to the station. In scenarios with several obstacles, the probability of finding an obstacle in the direct path from a sensor to the recharge station increase, so in these kind of scenarios, it is better to travel the graph.

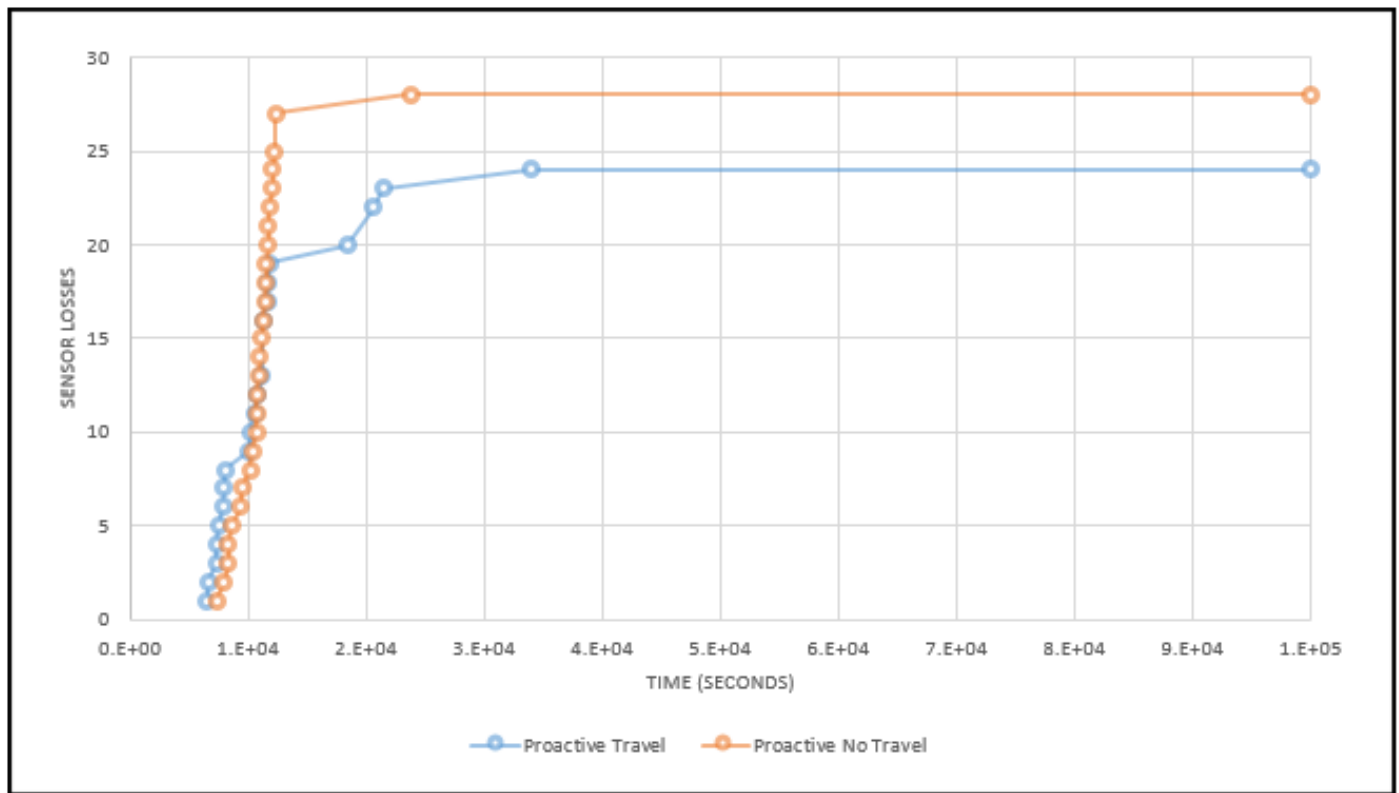

Figure 4.5: Travelling vs No Travelling the Graph.

Figure 4.6 shows how the number of obstacles impacts the results of the proactive strategy that travel and the proactive strategy that do not travel the graph. In Figure 4.6, it can be seen that in scenarios with less or equal than 3 obstacles it is better to travel directly 
to the recharge station, but when the number of obstacles is greater than 3 , it is better to travel the graph.

The results shown in Figure 4.5 and Figure 4.6 depend heavily on the type and number of obstacles. But in unknown environments where the number and type of obstacles are unknown, travelling the graph is the best option when the sensors are in panic run state. In this case, the sensors will have a safe path to the station and will avoid expending energy exploring obstacles while their batteries are critical.

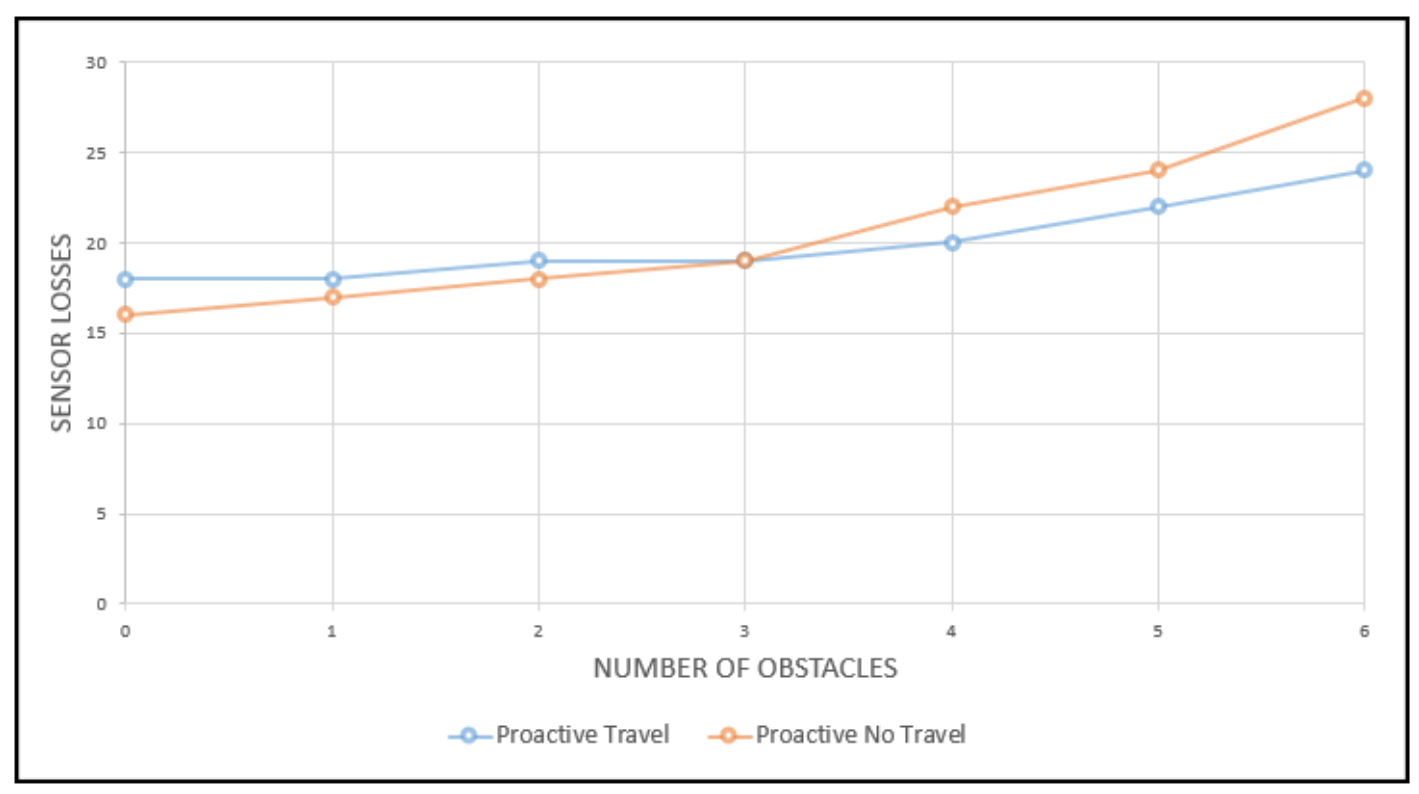

Figure 4.6: Impact of the Number of Obstacles.

\subsection{One-Hop Runs vs Panic Runs}

This experiment attempted to measure the number of panic runs vs the number of one-hop runs in each proactive solution proposed. In an ideal proactive strategy, all the recharges are done by sensors via one-hop runs to the recharge station (optimal trip). Figure 4.7 compares results for each proactive solution using the number of panic and the number of one-hop runs. The first result that can be seen in Figure 4.7 is that in all the proactive solutions the number of optimal trips to the recharge station is more than $60 \%$ of the total recharge trips. In Figure 4.7, it can also be seen that the proactive solutions that achieve the best results in term of optimal recharge (one-hop runs) are 
the ones where sensors do not travel the graph. Unfortunately, these solutions do not achieve the best results in term of sensor losses. The reason for this behaviour is because in solutions where sensors travel the graph more sensors survive, therefore there are more sensors trying to recharge their batteries at the same time, and demanding the limited positions at one-hop distance. Consequently, more recharge requests are done from other positions at greater distance (panic runs). The same behaviour is shown in the proactive solution that shares the information in comparison with the proactive solution that does not share the information about the obstacles. In the solution that share the information more sensor survive, therefore, there are more sensors trying to catch the positions at distance one-hop, at the same time. Due to the limited number of positions at one-ho distance, more requests are made from other positions (panic runs).

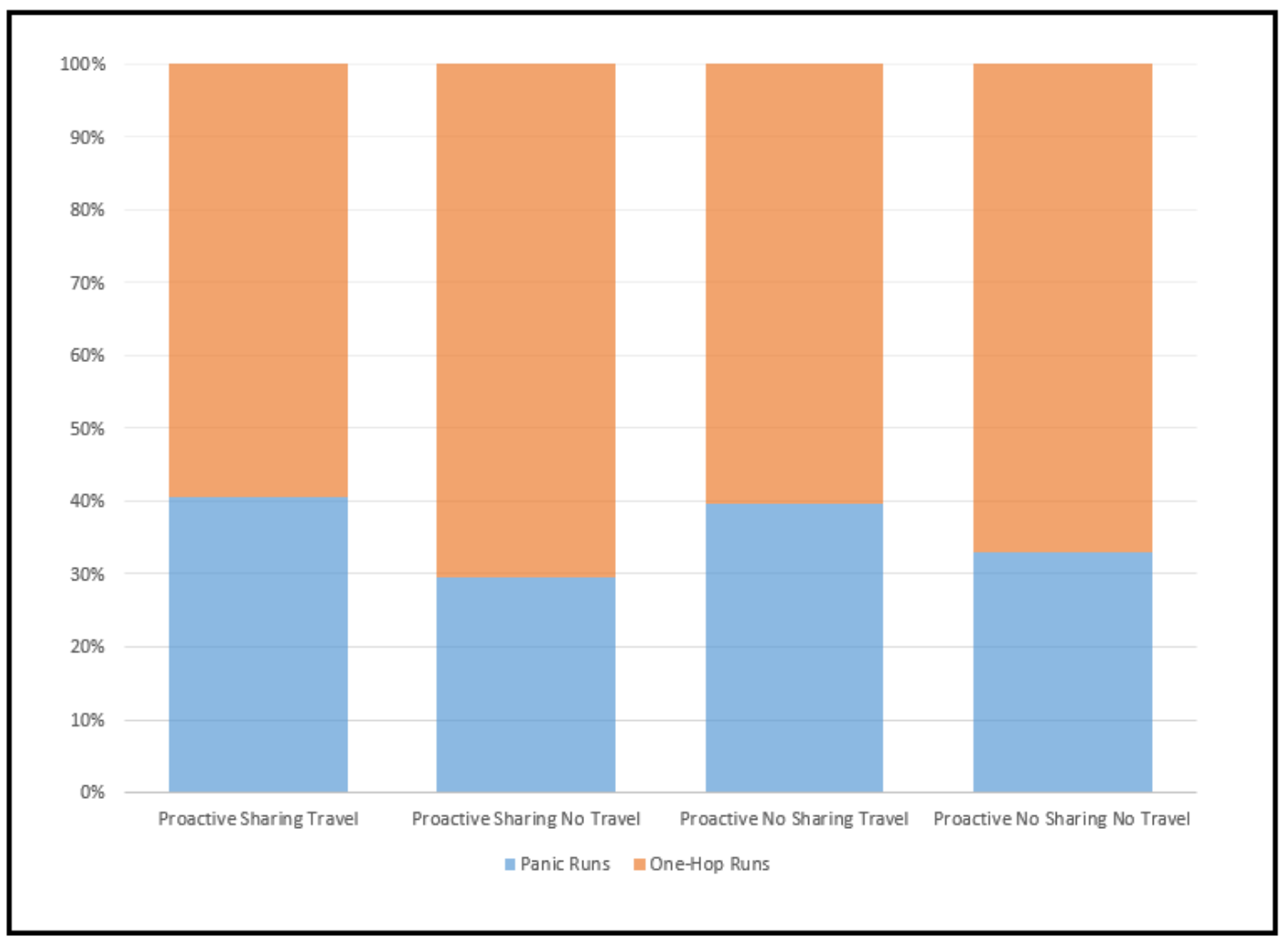

Figure 4.7: Panic Runs vs One-Hop Runs.

\subsection{Looking for the Ideal Energy Restoration}

With the idea of reaching the ideal energy restoration, different key parameters of the simulation were tested. The ideal energy restoration is the one where there are no sensor 
losses (perfect equilibrium).

\subsubsection{Increasing Sensor's Speed}

With the goal of finding a way to achieve the ideal energy restoration, it was decided to analyze the impact of the sensor's speed. Figure 4.8 shows different values of the sensor's speed. The results show that even with the maximum speed reached by modern mobile sensors (drones), the ideal energy restoration is not reached in our scenario.

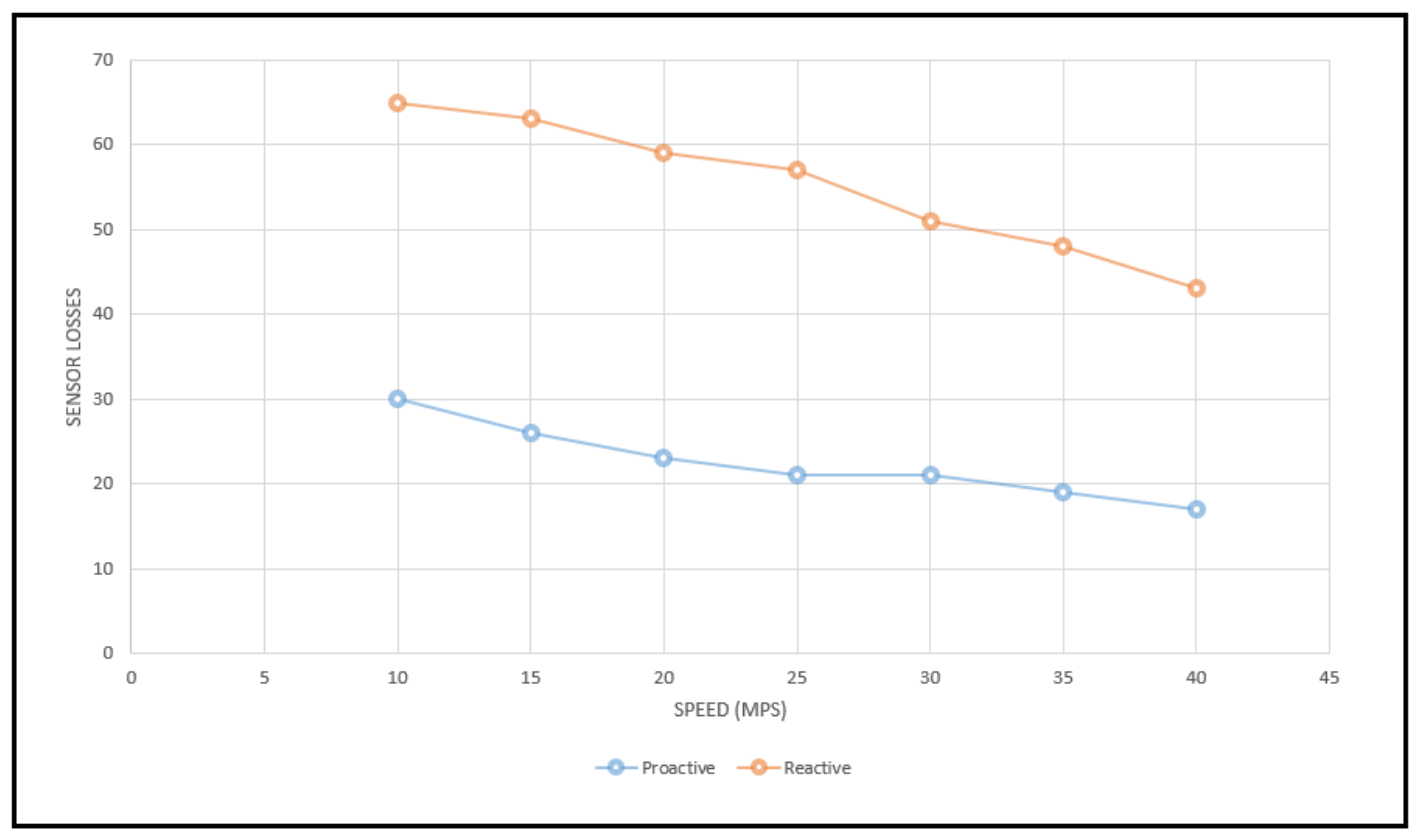

Figure 4.8: Speed of the Sensor.

\subsubsection{Increasing Number of Sockets}

In [25] the best improvement to achieve the ideal energy restoration was to increase the number of sockets of the recharge station. In [25], passive and proactive strategies reach the ideal state of equilibrium with 0 losses by increasing the number of sockets of the recharge station. In the scenarios where those results were accomplished, there were not obstacles.

In simulations experiments made in [25], the main cause of a depleted sensor's battery was the waiting time until a recharge socket was available. In some cases, the sensor's battery died before receive the RECHARGE_ACCEPT messages, and in other cases 
when the sensor received the RECHARGE_ACCEPT message the remaining battery was not enough to reach the station.

But in scenarios where there are obstacles, sensors can die while discovering the obstacles. Figure 4.9 shows how neither the passive, nor the proactive solution, reach the ideal equilibrium in the presence of obstacles. In both solutions, some sensors will die due to the obstacles. Then at some point, adding more sockets to the recharge station will not produce an improvement in order of sensor losses.

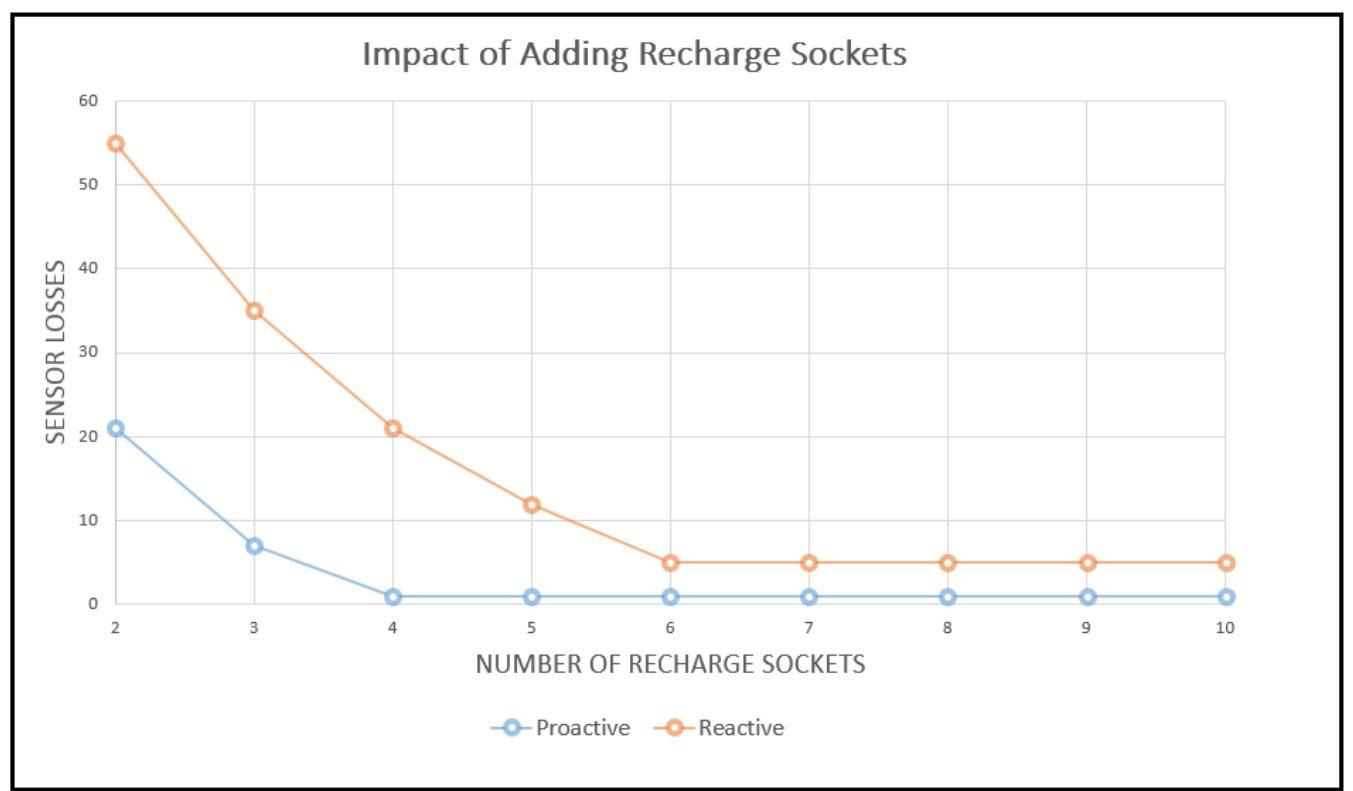

Figure 4.9: Impact of Increasing the Number of Sockets.

\subsubsection{Increasing the Value of the Recharge Threshold}

As it was mentioned before, the sensor's battery is depleted due to the waiting time until there is an available socket, or due to the obstacles perimeter is too large to be avoided with the sensor's battery in a BATTERY_CRITICAL state. By incrementing the number of sockets the waiting time cause of dead is eliminated. Then, it was decided to modify the value of the threshold $(\alpha)$ that decided when a sensor battery is critical, and need to be recharged. Increasing the value of the threshold allows sensors to have more battery energy when they need to reach the recharge station.

Figure 4.10 shows that the ideal energy restoration can be achieved by increasing the 
sensor's threshold $(\alpha)$ that decides when the sensor is in a BATTERY_CRITICAL state. This result was achieved with fixed number of sockets in the recharge station. The values were selected from the results shown in Figure 4.9: 4 sockets in the proactive, and 6 sockets in the passive strategy. It is important to remark that these results depend directly on the positions of the obstacles, their number, and their shape.

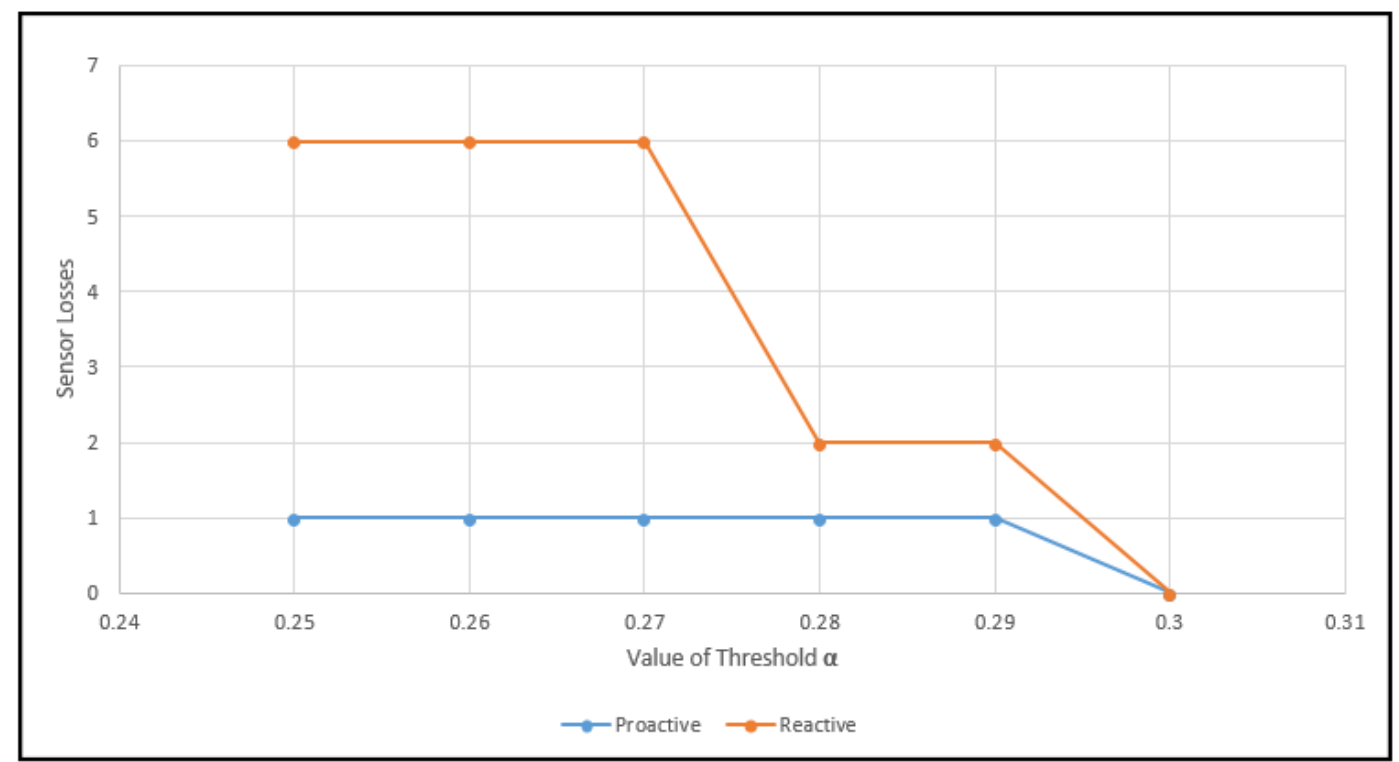

Figure 4.10: Impact of Increasing the Value of the Threshold $(\alpha)$.

\subsection{Proactive Strategies Comparison}

Finally, a performance comparison between all the proactive strategies was done. Figure 4.11 shows that the strategy that shares the frame of the obstacles and travels the graph $(\mathrm{CDG})$ when sensors are in panic run, achieves overall the best performance.

It is important to remark that these results were obtained by simulating different sensors deployments over the same scenarios, with the same obstacles. Obstacles' positions were the same for all the simulations done in this set of experiments. 


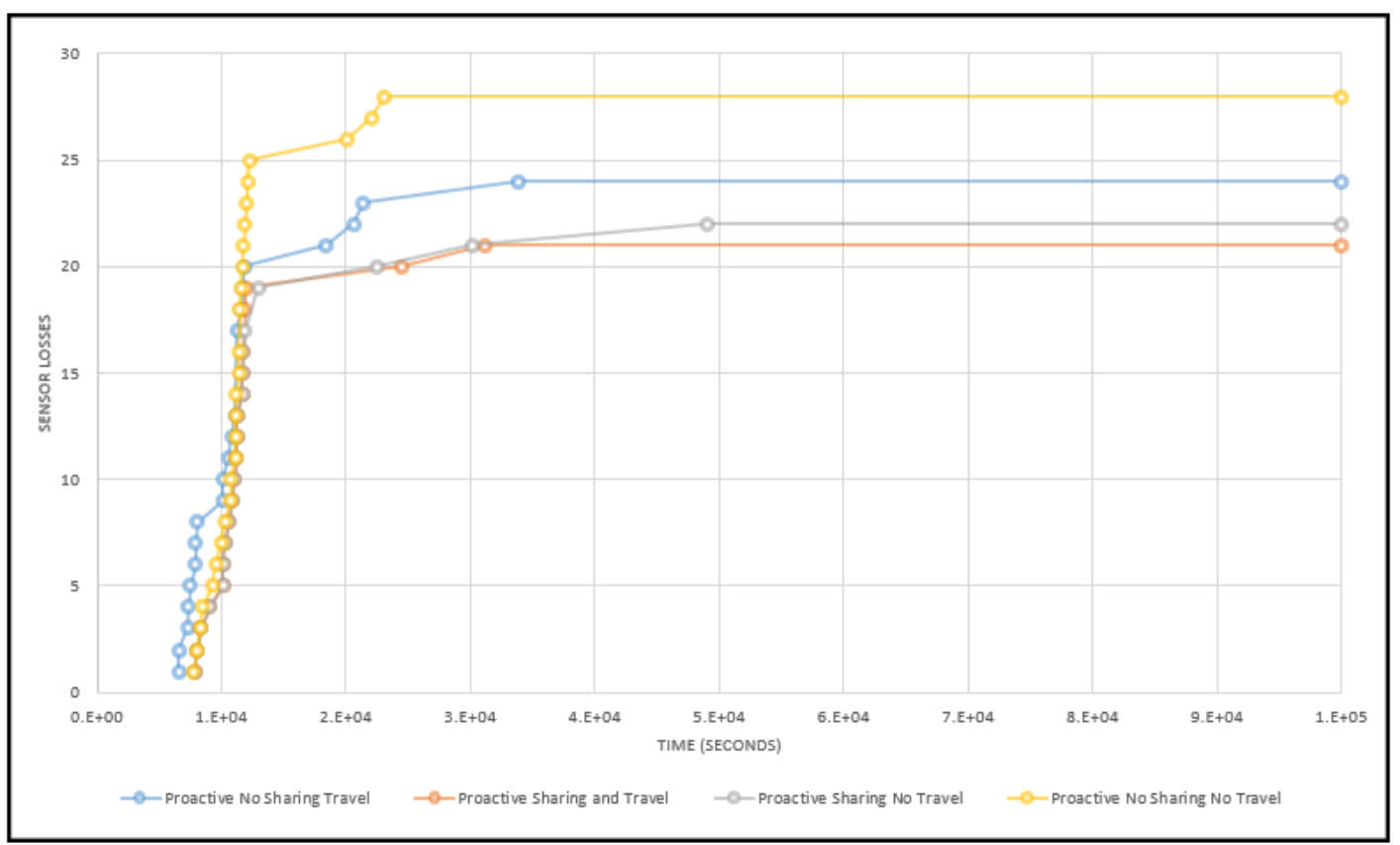

Figure 4.11: Proactive Strategies Comparison.

In addition, the number of obstacles used for these experiments was 6: 4 of them considered as large obstacles, and 2 small obstacles. The size of the obstacle has a direct impact on this results. However, in general unknown scenarios where it can be found all type of obstacles, the proactive strategy that shares and travels has better performance. On average, this strategy decreases the sensors losses in $20 \%$ in comparison with the strategy that does not travel the graph and does not share the obstacle information. In addition, as the number of obstacles and their size grows, the performance of the strategy that shares the obstacle information and travels the graph gets better in comparison with the others.

From the results shown in Figure 4.11, it can also be concluded that the second best technique is the proactive strategy that shares the obstacle's frame and does not travel the graph. The third best technique is the proactive strategy that does not share the information but travels the graph. Therefore, sharing the information has better benefit than travel the graph for the performance of the energy restoration strategy. 


\section{Chapter 5}

\section{Conclusions and Open Problems}

\subsection{Conclusions}

In this thesis, the problem of how to restore the energy in mobile sensor networks in the presence physical obstacles was analyzed. The main goal of any energy restoration strategy is to guarantee continuous operation without sensor losses. In scenarios with physical obstacles, some sensor losses will occur. Some sensors will die because of the obstacles, and others die because of the energy restoration strategy. After several experiments described in chapter 4, the present research found that there is a positive correlation between the number of physical obstacles in the scenarios and the number of sensors losses, with both proactive and passive energy restoration strategy. The degree of the impact of each obstacle depends on its perimeter, and its position.

To avoid obstacles efficiently, an obstacles avoidance technique based on the IBA algorithm was used, but with some modifications. The first modification introduced was to store and update the most extreme values of the discovered obstacles (Xmin, Xmax, Ymin, Ymax), and with them create rectangular frames containing the obstacles discovered. The frames serve as representations of the obstacles known by the sensors. By keeping only four values to represent each obstacle it is assured that the sensor memory will not be overloaded. With only these 4 values per obstacle, sensors can improve the efficiency of the paths used, and they can avoid unnecessary exploration.

The other modification made to the IBA algorithm was to count the turns sensors make 
while avoiding the obstacles. With the obstacle frame information and the turn count, sensors are able to avoid U- and H-shaped obstacles. In addition, this new characteristic allows sensors to solve polygonal mazes as long as the target position is outside the maze.

In order to not increase the energy expenditure, the sensors will use the same messages used to maintain and coordinate the energy restoration strategy to share the obstacles' frame information.

To analyze the impact of the obstacles and the impact of the obstacle avoidance technique used in the energy restoration strategies, two different strategies, passive and proactive, were evaluated. Both strategies are based on the ones presented in [35]. In both strategies, presented in the present research, the state of equilibrium was reached in the presence of obstacles. The behaviour of the strategies shown in [35] in scenarios without obstacles was the same as the behaviour observed that in our scenarios with obstacles. In both types of scenarios, the proactive strategy achieved much better results than the passive in terms of sensor losses, while the passive strategy achieved the state of equilibrium faster than the proactive. An interesting fact that was observed in the experiments is that in both strategies more than $85 \%$ of the sensor losses occur before those sensors can get their first recharge.

The experiments described in chapter 4 indicate that a proactive strategy that shares and uses the frames information to avoid obstacles achieves better results that a proactive strategy that does not share and use the frames. In contrast, sharing the frame information in the passive strategy led to worse results. The explanation of this behaviour is described in chapter 4 , section 4.4 .

Another finding in this thesis is that, in the proactive strategy, the best option when sensors need to travel to the recharge station is to travel the graph created by the energy restoration strategy. In unknown environments, moving directly to the recharge facility generally leads to worse results. The more obstacles there are, the higher the probability that travelling directly to the recharge station will lead to an obstacle, and will waste the sensor's precious time and energy trying to avoid it. 
Overall, the strategy that achieves the best performance in unknown environments containing obstacles is a proactive energy restoration strategy that shares the obstacles' frame information and travels the graph when it needs to recharge.

With the intention of finding the ideal energy restoration strategy, different key parameters were evaluated. It was proved that increasing the speed of the sensor, or increasing the number of sockets of the recharge station, do not, on their own, guarantee zero sensor losses. In scenarios without obstacles, increasing the number of sockets in the recharge station is the best solution to achieve the ideal restoration. The problem in scenarios with obstacles is that even when there is always a socket available for a sensor, if obstacles make the cost of the path to the recharge station greater than the energy the sensor has when its battery is critical, the sensor will always die. The only solution to this problem is to increase the value of the threshold $(\alpha)$ that defines when a battery is in a critical state.

In the experiment described in chapter 4 , subsection 4.7 .3 , that combine increasing the number of sockets and increasing the critical-energy-state threshold, the ideal equilibrium was reached. However, it is important to remark that the value of the threshold needed to achieve the ideal equilibrium depends on the perimeter of the obstacles and their number. Therefore in unknown environments, several simulations need to be done in order to adjust the value of the threshold to reach the ideal energy restoration. In addition, it is important to note that increasing only the threshold does not solve the problem because this reduces the amount of swapping operations and, therefore, the precision and accuracy of obstacle frames. Overly large frames lead the sensors to waste energy on long trips (as occurred with the passive strategy when sharing information). In addition, even if no sensors die due to obstacle avoidance, they will die due to the lack of recharge sockets, which leads to increased waiting time.

The importance of paying careful attention to all these factors is illustrated in Figure 5.1, which shows a worst case scenario for reaching the recharge facility. In the case shown in Figure 5.1, the sensor $A$ needs to recharge its battery in the early stages of the simulation. Therefore, the obstacles are unknown. Sensor $A$ needs to survive, an available socket at the recharge station, and it needs to have enough energy in BATTERY_CRITICAL state 
to avoid all the obstacles on the path to the station. In this case, having enough recharge sockets and having a high enough recharge threshold, is necessary to achieve ideal energy restoration.

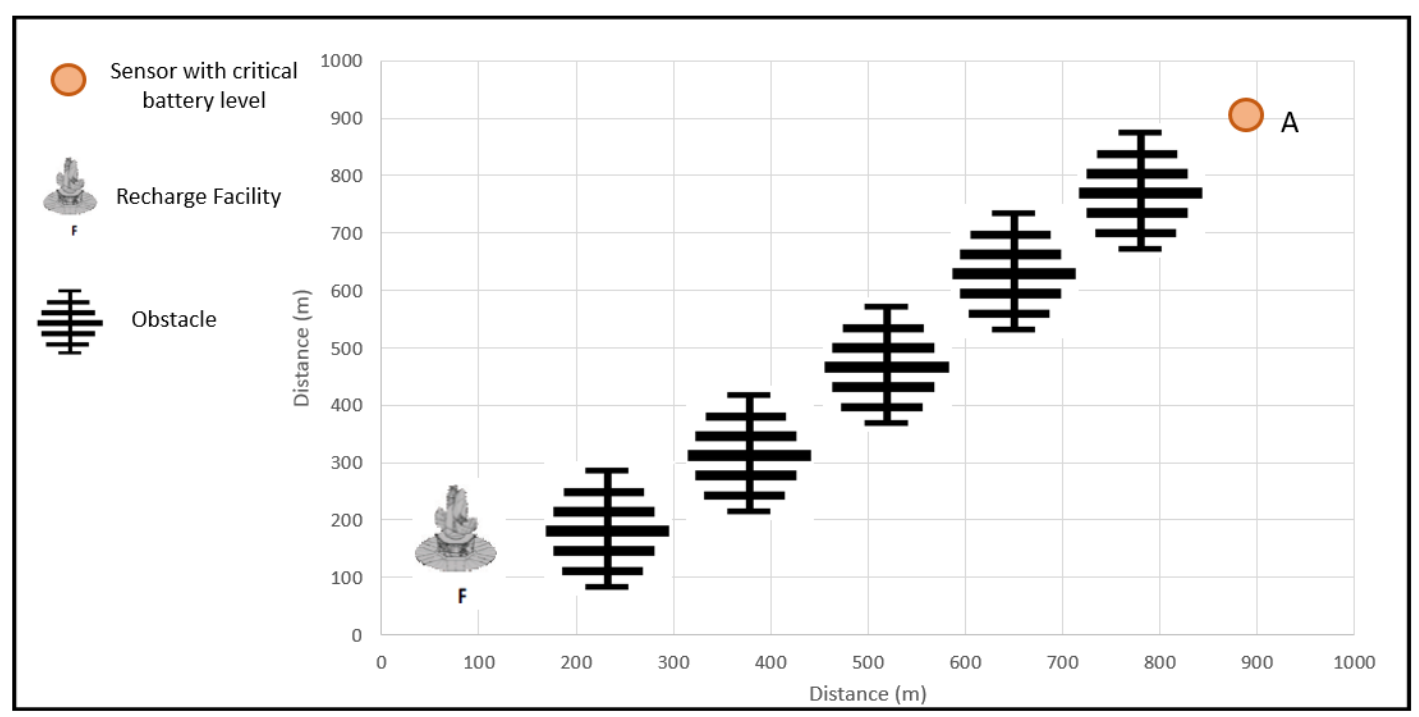

Figure 5.1: Worst-Case Scenario.

To validate the proposals presented in this thesis, more than 1640 experimental simulations were done. In addition, as part of the investigation, it was developed two OMNET ++ modules. The first module called IBAMMobility contains all the logic that a sensor needs to travel to a target position in scenarios with physical obstacles, and following the proposals presented in this thesis in chapter 3. The second module called ERS_app contains the logic needed to perform the energy restoration strategies presented in section 2.1 with the modifications proposed in chapter 3. Figure 5.2 shows a simple model of the sensors used in our experiments, and the two new modules implemented and added to the OMNET ++ libraries.

Finally, the results of this thesis can be applied in any scenario where mobile sensor networks are involved and there are physical obstacles. For example, in police surveillance, and in forest monitoring done by drones, this proactive solution to restore energy can be applied. It is only necessary to have a place where drones' batteries are replaced or recharged. The drones have to be programmed to fly at the same altitude, because the solution proposed in this thesis works on $\mathbb{R}^{2}$. Under those conditions, drones can restore their energy efficiently in scenarios that contain physical obstacles that have polygonal 
shapes or circular shapes.

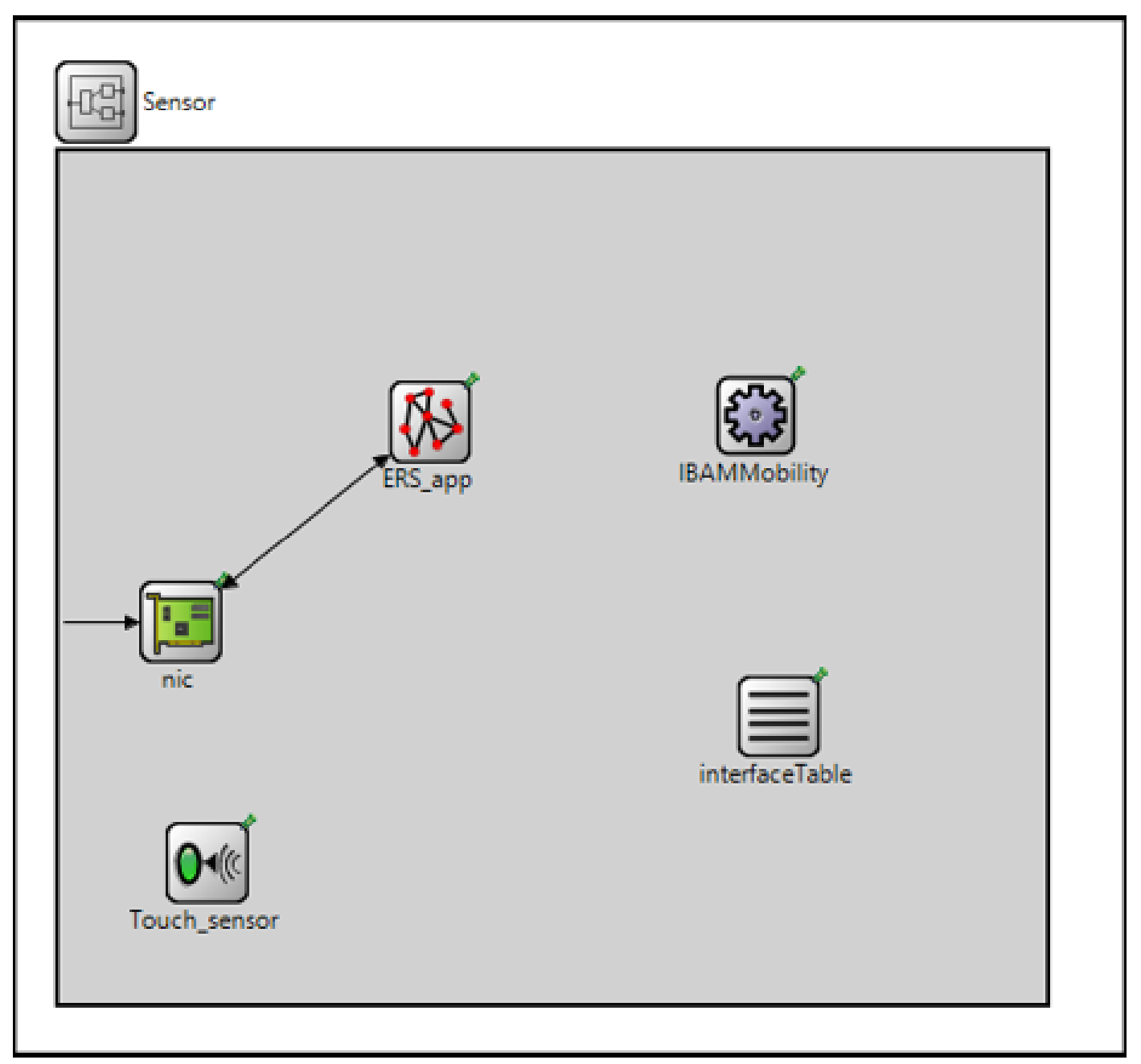

Figure 5.2: Model of a Sensor

\subsection{Open Problems}

\subsubsection{Self-Deployment}

Several important problems regarding energy restoration in mobile sensor networks in the presence of obstacles are still open and need to be addressed. The most challenging at present continues to be reducing the number of sensor losses. As was mentioned more than $85 \%$ of sensor losses in both strategies occur before the second recharge of any sensor. Before sensors get their first recharge, sensors are discovering the obstacles, therefore, the paths are not efficient. In our simulations, the deployment of the sensors 
was done using a random self-deployment algorithm, but if a method could be found for sensors to start gathering information about obstacles and paths during the process of self-deployment, this could help to generate obstacle frames, and improve paths faster. Therefore, sensors will reduce wasted time while discovering some obstacles.

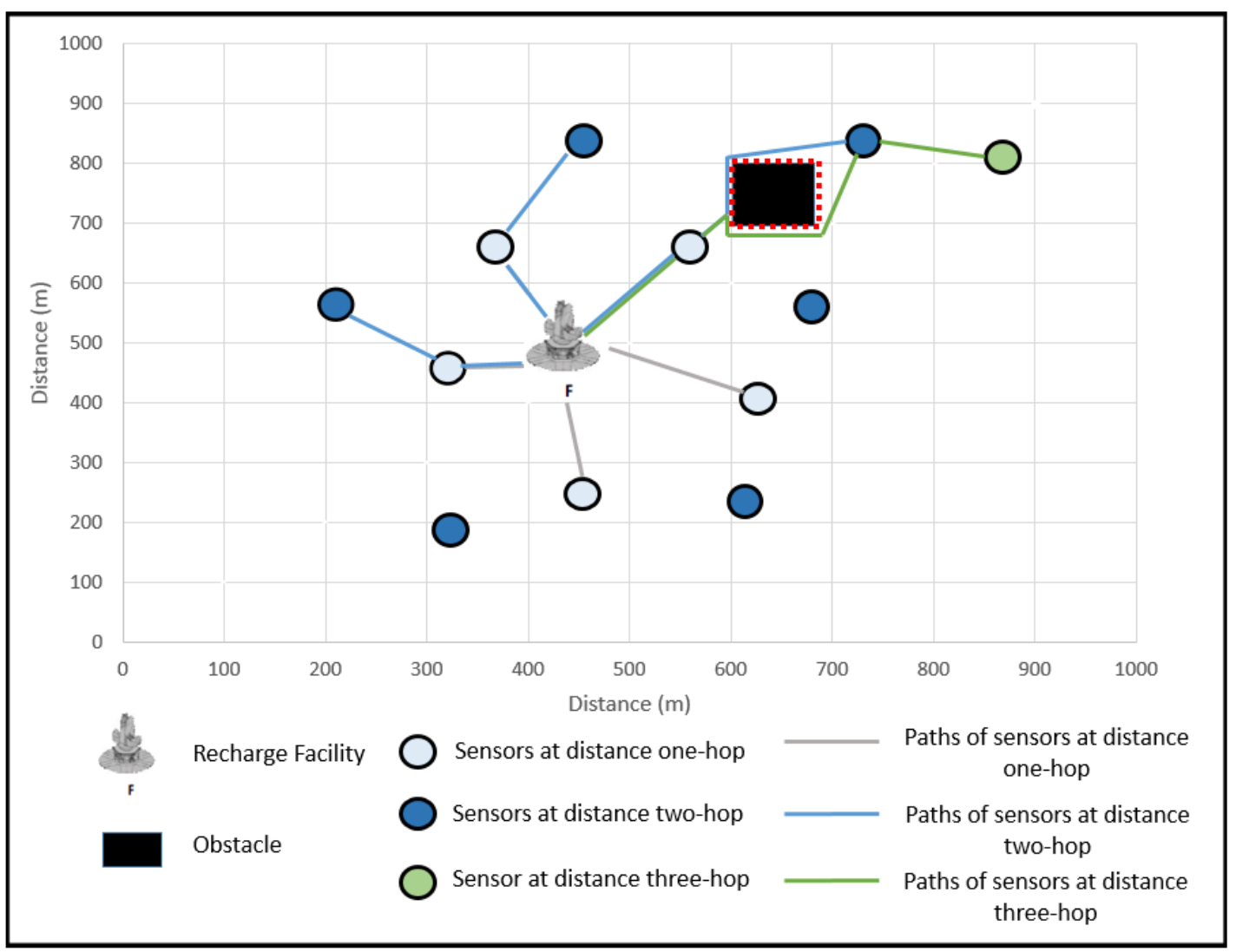

Figure 5.3: Self-Deployment Proposed.

For example, if all sensors began the self-deployment at the recharge station and know their final position, they could create the CDG at the station and start the deployment in order based on the hop distance to the station. The station could be the root of the graph (level 0), and the deployment would start for the neighbours at level 1 (one-hop distance). If these sensor found obstacles in their paths, they would always move clockwise to avoid the obstacles. Then when the sensors at level 2 (two-hop distance) had to travel to their deployment positions, they would travel first to their closest neighbours at level 1 , and then to their final positions at level 2 . If they reached obstacles when travelling to their neighbour's position, they will move counter-clockwise to avoid the obstacles. In this way, when they arrived at their neighbour's position, an 
obstacle's frame could be created even before the energy restoration strategy was started. Sensors at level $n$ would avoid obstacles moving counter-clockwise while travelling the path from the station to its closest neighbour (station, level 1, level 2, ... level $n-1)$, and they would move clockwise when travelling to it deployment position at level $n$.

After the self-deployment, the sensors would know a safe path to the station, a short path to its supposed closest neighbour, and in case there were obstacles on the paths to the station, they would know the frames of the obstacles. Sensors will need to spent some additional energy in communication to guarantee that the known paths are shared. Figure 5.3 shows how should be the self-deployment proposed. By collecting information about the environment in the self-deployment, sensors would improve paths faster, and therefore reduce the sensors death.

\subsubsection{Value of the Threshold $(\alpha)$}

In addition, the question of what would happen if the sensors more distant from the recharge station had a higher threshold to define the critical battery level deserve exploration. In the simulations, it was observed that the critical cases that produced sensors death while avoiding obstacles in the BATTERY_CRITICAL state were usually sensors that were far from the recharge station, and had several obstacles in their path to the recharge station. However, if they had higher battery levels, by the time they needed to recharge their batteries, two possible scenarios can occur. If the recharge needs to be done in the first moments of the simulation, with a high threshold the sensor guarantee be able to wait the RECHARGE_ACCEPT message, and then to travel the long path to the station, even when the obstacles on the path are unknown. Otherwise, if the recharge needs to be done after several stages of the simulation, several swaps would have occurred. Therefore, the obstacles should be known, and the sensor could generate an efficient path. In this case, sensor will need enough battery level to wait the RECHARGE_ACCEPT message, and then to travel to the station for a path that does not require so much energy as the previous case. The idea is find a mechanism that the threshold could change depending on the position of the sensor and the knowledge of the environment. 


\subsubsection{Communication}

In this thesis, it was assumed that obstacles were contained in a circle of radio $r=R / 2$. This was done to have an idea of how much time it would take for a sensor to avoid the complete circle that contains the obstacle. With this constraint, it was defined the waiting time for the SWAP_COMPLETE message response of a sensor. In the

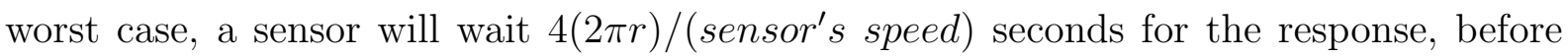
continuing with its performance. There are some extreme cases that can lead a sensor while swapping to a path longer than $4(2 \pi r)$ meters, therefore the other sensor involved in the swap do not wait enough. In other cases, the sensor dies or enters in panic run in a position that is out of the range of communication of the other sensor while swapping. Therefore, the other sensor spends time waiting for a response that it will never receive. In those cases, the question of which is the best waiting time for the sensor in the simulation, before continuing with its next operation, merits attention in future research. It would be useful to analyze how to calculate the best waiting time, and to see if the estimation can be done dynamically and based only on local information.

\subsubsection{Topology}

Another important area to explore is methods of graph creation that differ from Gabriel Neighbours and Relative Neighbours. During our simulations, it was observed that in scenarios where a sensor has more paths in the graph to reach different sensors at one-hop distance to the station in few swap, the number of sensor losses decrease. This means that sensors can reach different positions that are closer to the station with a few swap operations. In some scenarios where the number of positions at distance one-hop are few, or there is a bottleneck at these positions, there will be a high demand of swapping with the sensors at those positions. Therefore, a lot of sensors will have to travel to the station from other positions (panic runs), and they will have more probability of dying. The idea is to find a topology that prevents or reduces panic runs. 


\subsubsection{Complex Scenarios and Better Representations of the Obstacles}

A final proposal for future research relates to the problem of having more complicated obstacles (any shape), and developing better representations of them, while remaining conscious of the limited sensor memory. It would be useful to analyze if having better paths because of more accurate contour for an obstacle leads to better results. For example, by permitting sensors to chose between multiple common polygonal shapes for frames, like triangles, rectangles, and hexagons, depending on the obstacle discovered. It will be important to determinate if having more accurate obstacle frames is worth the cost in term of sensor memory. It is important to determinate how much memory we are willing to sacrifice in order to have a more real representation of the obstacles.

All these open problems and ideas should be explored, but always considering the main goals of the energy restoration strategies which are: extend the life of the sensors, and reach the state of equilibrium. 


\section{Bibliography}

[1] Harold Abelson and Andrea A. DiSessa. Turtle Geometry: The computer as a medium for exploring mathematics. MIT Press, 1980.

[2] Ludovic Apvrille, Tullio Tanzi, and Jean Luc Dugelay. Autonomous drones for assisting rescue services within the context of natural disasters. 31th URSI General Assembly and Scientific Symposium, pages 1-4, 2014.

[3] Mounir Arioua, Younes El Assari, Imad Ez-Zazi, and Ahmed El Oualkadi. Multi-hop cluster based routing approach for wireless sensor networks. Procedia Computer Science, 83:584-591, 2016.

[4] F. Arwin, K. Samsudin, and A. R. Ramli. Swarm robots long term autonomy using moveable charger. International Conference on Future Computer and Communication, pages 127-130, 2009.

[5] Matthew R. Burkhardt, Faranak Davoodi, Joel W. Burdick, and Farhooman Davoudi. Energy harvesting analysis for Moball, A self-propelled mobile sensor platform capable of long duration operation in harsh terrains. IEEE International Conference on Robotics and Automation, pages 2665-2672, 2014.

[6] Alessandra Capolupo, Stefania Pindozzi, Collins Okello, Nunzio Fiorentino, and Lorenzo Boccia. Photogrammetry for environmental monitoring: The use of drones and hydrological models for detection of soil contaminated by copper. Science of the Total Environment, 514:298-306, 2015.

[7] Chih Yung Chang, Chao Tsun Chang, Yu Chieh Chen, and Shih Chieh Lee. Active route-guiding protocols for resisting obstacles in wireless sensor networks. IEEE Transactions on Vehicular Technology, 59(9):4425-4442, 2010. 
[8] D. J. Dechene, A. El Jardali, M. Luccini, and A. Sauer. A survey of adaptive distributed clustering algorithms for wireless sensor networks. International Journal of Computer Science \& Engineering Survey, 2(4):165-176, 2011.

[9] Jakob Gakkestad and Leif Hanssen. Powering wireless sensor networks nodes in northern europe using solar cell panel for energy harvesting. 4th IFIP International Conference on New Technologies, Mobility and Security, pages 1-5, 2011.

[10] Hari Prabhat Gupta, S. V. Rao, Amit Kumar Yadav, and Tanima Dutta. Geographic routing in clustered wireless sensor networks among obstacles. IEEE Sensors Journal, 15(5):2984-2992, 2015.

[11] Essia Hamouda, Nathalie Mitton, Bogdan Pavkovic, and David Simplot-Ryl. Energy-aware georouting with guaranteed delivery in wireless sensor networks with obstacles. International Journal of Wireless Information Networks, 16(3):142-153, 2009.

[12] Sunghyuck Hong and Kun Hee Han. Cost-Efficient Routing Protocol (CERP) on wireless sensor networks . Wireless Personal Communications, 79(4):2517-2530, 2014.

[13] Tom Kamphans and Elmar Langetepe. The Pledge Algorithm reconsidered under errors in sensors and motion. 1st Workshop on Approximation and Online Algorithms, pages 165-178, 2004.

[14] Aman Kansal and Mani B. M.B. Srivastava. An environmental energy harvesting framework for sensor networks. International Symposium on Low Power Electronics and Design (ISLPED), pages 481-486, 2003.

[15] Parul Khurana. Wireless sensor network routing protocols : A survey. International Journal of Computer Applications, 75(15):17-25, 2013.

[16] S.L. Laubach and J.W. Burdick. An autonomous sensor-based path-planner for planetary microrovers. IEEE International Conference on Robotics and Automation, 1:347-354, 1999 . 
[17] Yaroslav Litus, Richard T. Vaughan, and Pawel Zebrowski. The frugal feeding problem: Energy-efficient, multi-robot, multi-place rendezvous. IEEE International Conference on Robotics and Automation (ICRA), pages 27-32, 2007.

[18] James Ng and Thomas Bräunl. Performance comparison of Bug navigation algorithms. Journal of Intelligent and Robotic Systems: Theory and Applications, $50(1): 73-84,2007$.

[19] Mohd Fauzi Othman and Khairunnisa Shazali. Wireless sensor network applications: A study in environment monitoring system. International Symposium on Robotics and Intelligent Sensors (IRIS), 41:1204-1210, 2012.

[20] Jaime Paneque-Gálvez, Michael K. McCall, Brian M. Napoletano, Serge A. Wich, and Lian Pin Koh. Small drones for community-based forest monitoring: An assessment of their feasibility and potential in tropical areas. Forests, 5(6):1481-1507, 2014.

[21] Prasad Poonam. Recent trend in wireless sensor network and its applications : A survey. Sensor Review, 35(2):229-236, 2015.

[22] Bushra Rashid and Mubashir Husain Rehmani. Applications of wireless sensor networks for urban areas: A survey. Journal of Network and Computer Applications, 60:192-219, 2016.

[23] Mrutyunjay Rout and Rajarshi Roy. Dynamic deployment of randomly deployed mobile sensor nodes in the presence of obstacles. Ad Hoc Networks, 46:12-22, 2016.

[24] Mrutyunjay Rout and Rajarshi Roy. Self-deployment of mobile sensors to achieve target coverage in the presence of obstacles. IEEE Sensors Journal, 16(14):5837-5842, 2016.

[25] Nicola Santoro and Elio Velazquez. Energy restoration in mobile sensor networks. In Chapter 6 of: Wireless Sensor and Robot Networks, pages 113-142. World Scientific Publishing Co. Pte. Ltd., 2014.

[26] Getsy S. Sara and D. Sridharan. Routing in mobile wireless sensor network: A survey. Telecommunication Systems, 57(1):51-79, 2014. 
[27] Bryan Sarazin and Syed S Rizvi. A Self-deployment Obstacle Avoidance (SOA) algorithm for mobile sensor networks. International Journal of Computer Science and Security (IJCSS), 4(3):316-330, 2010.

[28] Volkan Sezer and Metin Gokasan. A novel obstacle avoidance algorithm: Follow the gap method. Robotics and Autonomous Systems, 60(9):1123-1134, 2012.

[29] Faisal Karim Shaikh and Sherali Zeadally. Energy harvesting in wireless sensor networks: A comprehensive review. Renewable and Sustainable Energy Reviews, 55:1041-1054, 2016.

[30] Mohsen Sharifi, Saeed Sedighian, and Maryam Kamali. Recharging sensor nodes using implicit actor coordination in wireless sensor actor networks. Wireless Sensor Network, 2(2):123-128, 2010.

[31] Junji Takahashi, Kosuke Sekiyama, and Toshio Fukuda. Self-deployment algorithm for mobile sensor network. IEEE International Conference on Robotics and Biomimetics, pages 1434-1439, 2008.

[32] C. Urdiales, F. Aguilera, E. González-Parada, J. Cano-García, and F. Sandoval. Rule-based vs. Behavior-based self-deployment for mobile wireless sensor networks. Sensors, 16(7), 2016.

[33] Andras Varga. The omnet++ discrete event simulation system. European Simulation Multi-conference (ESM), pages 319-324, 2001.

[34] Andras Varga, Zoltan Bojthe, Levente Meszaros, Benjamin Seregi, and Rudolf Hornig. INET framework for OMNeT++/OMNEST 3.2.0. An open-source OMNeT ++ model suite for wired, wireless and mobile networks, 2015. Available at https://inet.omnetpp.org/, version 3.2.0.

[35] Elio Velazquez and Nicola Santoro. Mobility-based strategies for energy restoration in Wireless Sensor Networks. 6th International Conference on Mobile Ad-hoc and Sensor Networks, pages 161-168, 2010.

[36] Elio Velazquez, Nicola Santoro, and Mark Lanthier. Pro-active strategies for the frugal feeding problem in wireless sensor networks. 2nd International Conference on Sensor Systems and Software, pages 189-204, 2010. 
[37] Daniele Ventura, Michele Bruno, Giovanna Jona Lasinio, Andrea Belluscio, and Giandomenico Ardizzone. A low-cost drone based application for identifying and mapping of coastal fish nursery grounds. Estuarine, Coastal and Shelf Science, 171:85-98, 2016.

[38] Jens Wawerla and Richard T Vaughan. Near-optimal mobile robot recharging with the rate-maximizing forager. Artificial Life, pages 776-785, 2007.

[39] Dali Wei, Yichao Jin, Serdar Vural, Klaus Moessner, and Rahim Tafazolli. An energy-efficient clustering solution for wireless sensor networks. IEEE Transactions on Wireless Communications, 10(11):3973-3983, 2011.

[40] Liguang Xie, Yi Shi, Y .Thomas Hou, and Hanif D Sherali. Making sensor networks immortal : wireless power transfer. IEEE/ACM Transactions on Networking, 20(6):1748-1761, 2012.

[41] Alpaslan Yufka and Osman Parlaktuna. Performance comparison of BUG algorithms for mobile robots. 5th International Advanced Technologies Symposium (IATS), pages 61-65, 2009.

[42] A. S M Zahid Kausar, Ahmed Wasif Reza, Mashad Uddin Saleh, and Harikrishnan Ramiah. Energizing wireless sensor networks by energy harvesting systems: Scopes, challenges and approaches. Renewable and Sustainable Energy Reviews, 38:973-989, 2014.

[43] Jian Zhang, Jianbo Hu, Juyu Lian, Zongji Fan, Xuejun Ouyang, and Wanhui Ye. Seeing the forest from drones: Testing the potential of lightweight drones as a tool for long-term forest monitoring. Biological Conservation, 198:60-69, 2016.

[44] Muhammad Zohaib, M. Pasha, R. A. Riaz, Nadeem Javaid, M. Ilahi, and R. D. Khan. Control strategies for mobile robot with obstacle avoidance. Journal of Basic and Applied Scientific Research (JBASR), 3(4):1027-1036, 2013.

[45] Muhammad Zohaib, Syed Mustafa Pasha, Nadeem Javaid, and Jamshed Iqbal. IBA: Intelligent Bug Algorithm - A novel strategy to navigate mobile robots autonomously. Communication Technologies, Information Security and Sustainable Development: 3rd International Multi-topic Conference, (IMTIC), pages 291-299, 2013. 
[46] Muhammad Zohaib, Syed Mustafa Pasha, Nadeem Javaid, Jamshed Iqbal, and Abdus Salaam. An improved algorithm for collision avoidance in environments having $\mathrm{U}$ and H shaped obstacles. Studies in Informatics and Control, 23(1):97-106, 2014. 